Land Change Science Program

\title{
Social Values for Ecosystem Services, Version 4.0 (SoIVES 4.0)—Documentation and User Manual
}

Chapter 25 of

Section C, Computer Programs, of

Book 7, Automated Data Processing and Computations

Techniques and Methods 7-C25 



\section{Social Values for Ecosystem Services, Version 4.0 (SoIVES 4.0)-Documentation and User Manual}

By Benson C. Sherrouse and Darius J. Semmens

Chapter 25 of

Section C, Computer Programs, of

Book 7, Automated Data Processing and Computations

Land Change Science Program

Techniques and Methods 7-C25 


\title{
U.S. Department of the Interior \\ DAVID BERNHARDT, Secretary
}

\author{
U.S. Geological Survey \\ James F. Reilly II, Director
}

U.S. Geological Survey, Reston, Virginia: 2020

For more information on the USGS - the Federal source for science about the Earth, its natural and living resources, natural hazards, and the environment—visit https://www.usgs.gov or call 1-888-ASK-USGS.

For an overview of USGS information products, including maps, imagery, and publications, visit https://store.usgs.gov/.

Any use of trade, firm, or product names is for descriptive purposes only and does not imply endorsement by the U.S. Government.

Although this information product, for the most part, is in the public domain, it also may contain copyrighted materials as noted in the text. Permission to reproduce copyrighted items must be secured from the copyright owner.

Although this software program has been used by the U.S. Geological Survey (USGS), no warranty, expressed or implied, is made by the USGS or the U.S. Government as to the accuracy and functioning of the program and related program material nor shall the fact of distribution constitute any such warranty, and no responsibility is assumed by the USGS in connection therewith. Social Values for Ecosystem Services (SoIVES) is a tool for mapping and analyzing social survey response data. It is not a tool designed for the collection of survey data, nor is any survey attached to SoIVES. Any survey or survey response data referred to in the SoIVES documentation, sample data, or publications are the work and responsibility of the persons or groups who developed and performed that survey. Please note that before a Federal agency may collect information or sponsor a collection of information, the Paperwork Reduction Act of 1995 requires approval from the Office of Management and Budget. Any Federal agency or sponsored program interested in developing and performing a survey for use with SoIVES is responsible for submitting an Information Collection Request to the Office of Management and Budget.

Suggested citation:

Sherrouse, B.C., and Semmens, D.J., 2020, Social Values for Ecosystem Services, version 4.0 (SolVES 4.0)— Documentation and user manual: U.S. Geological Survey Techniques and Methods, book 7, chap. C25, 59 p., https://doi.org/10.3133/tm7C25.

ISSN 2328-7055 (online) 


\section{Acknowledgments}

Social Values for Ecosystem Services (SolVES) was originally developed in collaboration with Dr. Jessica M. Western (Clement) of the Ruckelshaus Institute of the University of Wyoming. Dr. Western shared survey data collected from nearby residents of the Pike and San Isabel National Forests while at Colorado State University that served as the basis for the initial development of SoIVES. Thanks are extended to Sabin Ray of the World Resources Institute Cities4Forests Initiative, as well as Dr. Carena J. van Riper and Evan L. Salcido of the University of Illinois Department of Natural Resources and Environmental Sciences who reviewed the SoIVES 4.0 software and documentation.

Several U.S. Geological Survey staff members are very much appreciated for their assistance during the SoIVES 4.0 development and release process. Zachary H. Ancona completed numerous rounds of end-user testing, Jodi L. Riegle completed the code review, and Joshua D. Takacs completed the administrative security review. 



\section{Contents}

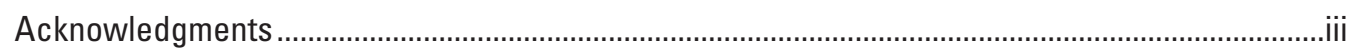

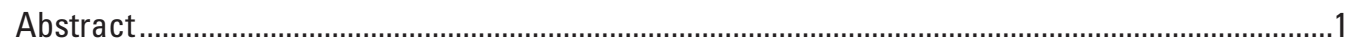

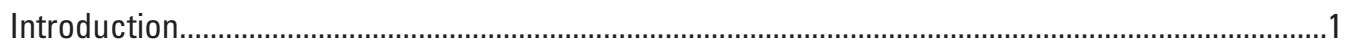

Maxent Maximum Entropy Modeling Software ...................................................................

New in SoIVES 4.0

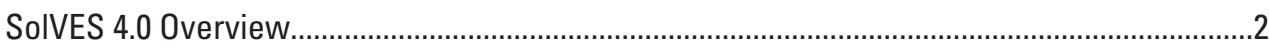

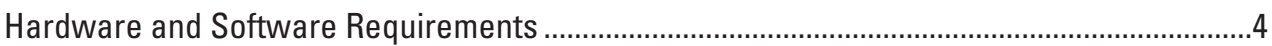

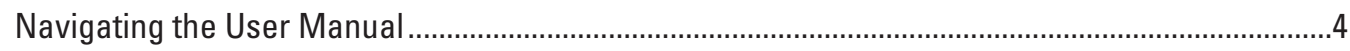

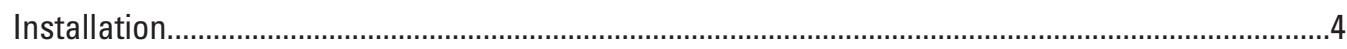

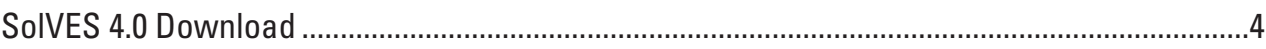

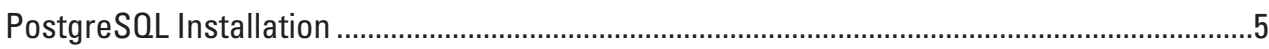

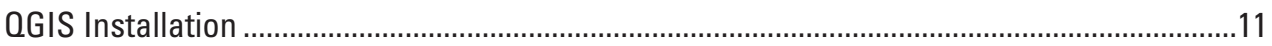

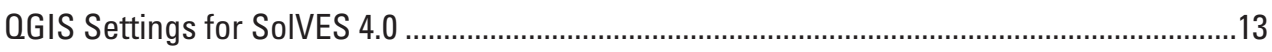

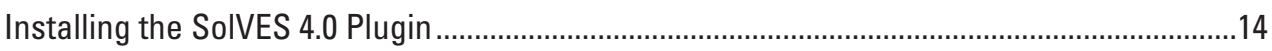

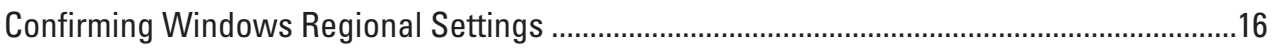

Sample Data Installation ..................................................................................................

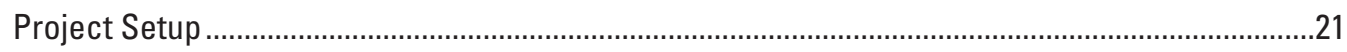

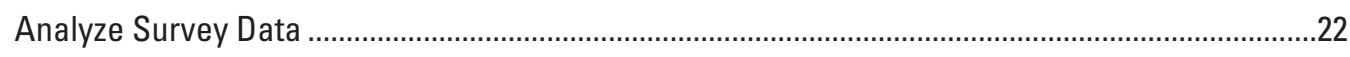

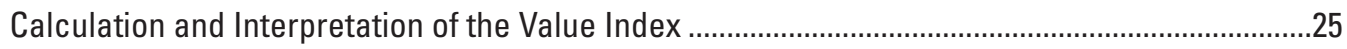

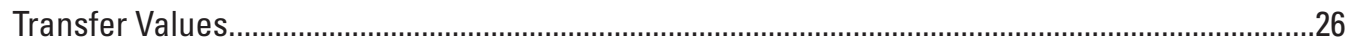

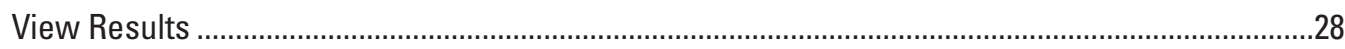

Interpreting and Adjusting Maxent's Area Under the Curve Values and Variable

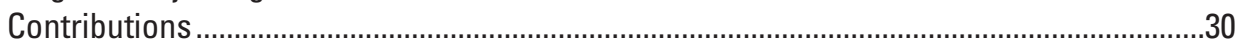

Digitizing Points Mapped by Survey Respondents …….............................................................

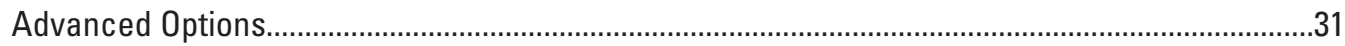

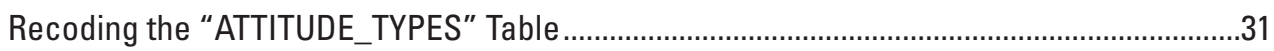

Substituting the Public Use Parameter .........................................................................................32

Creating Survey Data Subgroups Before Loading SolVES .....................................................32

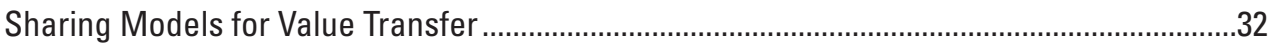

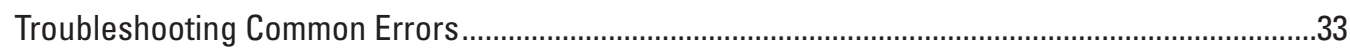

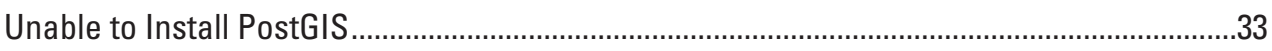

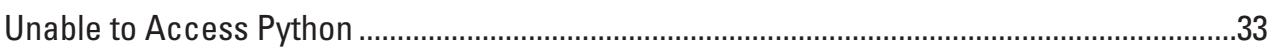

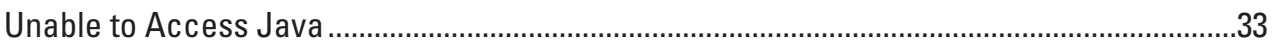

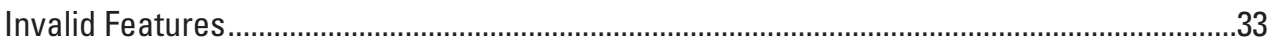

Environmental Data Not Found ..............................................................................................33

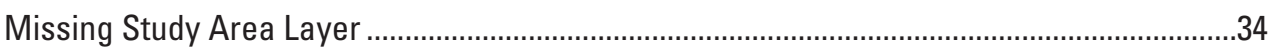

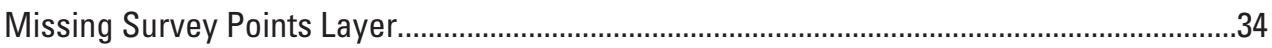

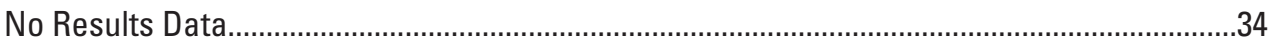

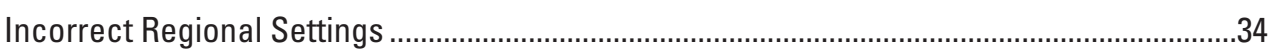

Incorrect Text Size ........................................................................................................

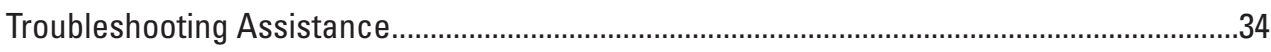

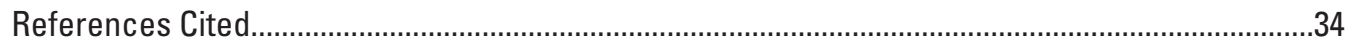

Appendix 1. Social Values for Ecosystem Services, Version 4.0, Data Requirements,

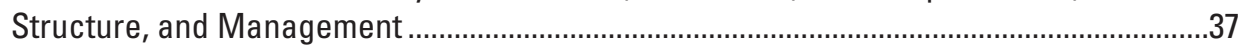


Appendix 2. Preparing and Loading User-Supplied Data to the "solves" Database .....................44

Appendix 3. Social Values for Ecosystem Services, Version 4.0, Data Dictionary.

\section{Figures}

1. Screenshot showing the generalized Social Values for Ecosystem Services, version 4.0, process flow.

2. Screenshot showing the SoIVES top-level directory structure after unzipping the "SolVES_V4.zip" file

3. Screenshot showing the location of the PostgreSOL installation file .................................5

4. Screenshot showing the PostgreSQL setup screen........................................................5

5. Screenshot showing the PostgreSQL 11.7 installation directory screen ...........................5

6. Screenshot showing the PostgreSOL 11.7 select components screen .............................5

7. Screenshot showing the PostgreSQL 11.7 data directory screen ....................................6

8. Screenshot showing the PostgreSOL 11.7 password screen ..............................................

9. Screenshot showing the PostgreSQL 11.7 port screen .................................................6

10. Screenshot showing the PostgreSOL 11.7 advanced options screen................................

11. Screenshot showing the PostgreSOL 11.7 pre-installation summary screen ....................7

12. Screenshot showing the PostgreSOL 11.7 ready to install screen ...................................

13. Screenshot showing the PostgreSOL 11.7 installation in progress ...................................

14. Screenshot showing the completing the PostgreSOL 11.7 setup wizard screen ..............7

15. Screenshot showing the Stack Builder 4.1 .0 welcome screen .......................................

16. Screenshot showing the PostGIS 2.5.3 selection screen of Stack Builder 4.1.0 ..............8

17. Screenshot showing the selected packages screen of Stack Builder 4.1.0 …..................8

18. Screenshot showing the license agreement screen for PostGIS 2.5.3 …........................8

19. Screenshot showing the confirm PostGIS 2.5.3 installation screen of Stack

Builder 4.1.0

20. Screenshot showing the PostGIS 2.5 .3 choose components screen .................................

21. Screenshot showing the PostGIS 2.5.3 installation location screen .................................9

22. Screenshot showing the PostGIS 2.5 .3 installation in progress ........................................

23. Screenshot showing the PostGIS 2.5.3 environmental variable and driver enabling message screens ....................................................................................10

24. Screenshot showing the PostGIS 2.5.3 installation complete screen .............................10

25. Screenshot showing the PostGIS 2.5.3 installation completed screen of Stack Builder 4.1.0 . .10

26. Screenshot showing the location of the QGIS 3.8.2 installation file .............................11

27. Screenshot showing the QGIS 3.8.2 setup wizard screen............................................11

28. Screenshot showing the OGIS 3.8.2 license agreement screen ....................................11

29. Screenshot showing the QGIS 3.8.2 choose install location screen ...............................11

30. Screenshot showing the QGIS 3.8 .2 choose components screen ..................................12

31. Screenshot showing the QGIS 3.8.2 installation in progress .....................................12

32. Screenshot showing the QGIS 3.8.2 setup completion screen......................................12

33. Screenshot showing the QGIS 3.8.2 welcome screen ...................................................13

34. Screenshot showing modifying QGIS 3.8 .2 environment variables .................................13

35. Screenshot showing setting the invalid features filtering value in QGIS 3.8.2 ...............14 
36. Screenshot showing the QGIS 3.8.2 plugins install from ZIP screen..............................14

37. Screenshot showing the location of the "solves.zip" plugin installation file ..................14

38. Screenshot showing the install plugin selected "solves.zip" file in QGIS 3.8.2..............15

39. Screenshot showing the plugin installation warning screen of QGIS 3.8.2 .....................15

40. Screenshot showing the successful installation message of QGIS 3.8.2 …..................15

41. Screenshot showing the successfully installed SoIVES plugin.....................................15

42. Screenshot showing selecting visibility of SoIVES plugin in QGIS $3.8 .2 \ldots \ldots \ldots \ldots \ldots \ldots \ldots \ldots . .15$

43. Screenshot showing accessing the Windows Control Panel using the Windows

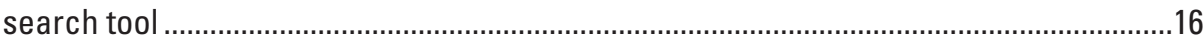

44. Screenshot showing selecting the Windows clock and region settings........................16

45. Screenshot showing selecting the "Change date, time, or number formats" option in Windows settings...

46. Screenshot showing selecting "Additional settings..." on the Windows region screen

47. Screenshot showing the numbers format for SoIVES processing in Windows settings .17

48. Screenshot showing accessing pgAdmin 4 using the Windows search tool..................17

49. Screenshot showing entering a pgAdmin 4 master password........................................17

50. Screenshot showing entering the "postgres" password in pgAdmin 4..........................18

51. Screenshot showing creating database in PostgreSOL 11, pgAdmin 4 ….....................18

52. Screenshot showing creating the "solves" database in pgAdmin 4 ..............................18

53. Screenshot showing setting the "solves" database definition in pgAdmin 4 .................18

54. Screenshot showing the restore data to "solves" database in pgAdmin 4 ...................19

55. Screenshot showing the database restore screen of pgAdmin 4...................................19

56. screenshot showing selecting the "solves.backup" file in pgAdmin 4...........................19

57. Screenshot showing the database restore screen with selected "solves. backup" file in pgAdmin 4 ........................................................................................19

58. Screenshot showing the successful completion of "solves" database

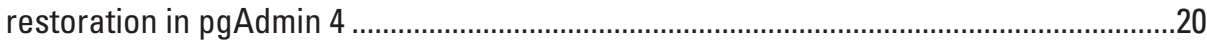

59. Screenshot showing the delete/drop in the "solves" database in pgAdmin 4 ...............20

60. Screenshot showing the confirm deletion of existing "solves" database in pgAdmin 4

61. Screenshot showing creating a new project in Social Values for Ecosystem

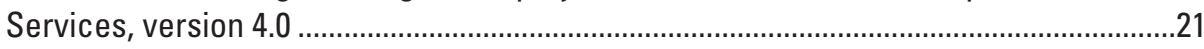

62. Screenshot showing the Social Values for Ecosystem Services, version 4.0, project setup form

63. Screenshot showing navigating to the Social Values for Ecosystem Services, version 4.0, directory from the project setup form

64. Screenshot showing the populated Social Values for Ecosystem Services, version 4.0, project setup form

65. Screenshot showing the Analyze Survey Data tool of Social Values for Ecosystem Services, version 4.0.

66. Screenshot showing the Analyze Survey Data tool ready to process the Ecosystem Services Social-Values Model of Social Values for Ecosystem Services, version 4.0

67. Screenshot showing the Ecosystem Services Social-Values Model generalized process flow of Social Values for Ecosystem Services, version 4.0. 
68. Screenshot showing the Analyze Survey Data tool ready for processing the Value Mapping Model of Social Values for Ecosystem Services, version 4.0

69. Screenshot showing the Value Mapping Model generalized process flow of Social Values for Ecosystem Services, version 4.0.

70. Screenshot showing an example of survey subgroups when the Analyze Survey Data tool "By Social Value Type Across Survey Subgroups" value comparison type is used in Social Values for Ecosystem Services, version 4.0

71. Screenshot showing navigating to the location of the models and metadata for use with the Transfer Values tool.

72. Screenshot showing the partial contents of a value transfer model metadata file ........26

73. Screenshot showing setting up a transfer values project in Social Values for Ecosystem Services, version 4.0.

74. Screenshot showing the Transfer Values tool of Social Values for Ecosystem Services, version 4.0

75. Screenshot showing the Transfer Values tool screen after parameter selection in Social Values for Ecosystem Services, version 4.0

76. Screenshot showing the Value Transfer Mapping Model generalized process flow of Social Values for Ecosystem Services, version 4.0.

77. Screenshot showing selecting the View Results tool in Social Values for Ecosystem Services, version 4.0.

78. Screenshot showing accessing and populating the View Results tool in Social Values for Ecosystem Services, version 4.0.

79. Screenshot showing the view results from the Analyze Survey Data tool in Social Values for Ecosystem Services, version 4.0.

80. Screenshot showing the view results from the Transfer Values tool in Social Values for Ecosystem Services, version 4.0.

81. Screenshot showing an example of Receiver Operating Characteristic curve and associated Area Under the Curve statistics generated by Maxent.

82. Screenshot showing an example jackknife graph of test Area Under the Curve statistics generated by Maxent.

83. Screenshot showing an example of variable contributions included in Maxent's HyperText Markup Language output.

84. Screenshot showing an example of a recoded "ATTITUDE_TYPES" table as viewed with pgAdmin 4

85. Screenshot showing the warning and error messages received during PostGIS 2.5.3 installation

86. Screenshot showing the location of the PostGIS 2.5 .3 installation file. 


\section{Abbreviations}

$\begin{array}{ll}\text { ASCII } & \text { American Standard Code for Information Interchange } \\ \text { AUC } & \text { Area Under the Curve } \\ \text { CSV } & \text { comma-separated values } \\ \text { GIS } & \text { geographic information system } \\ \text { HTML } & \text { Hypertext Markup Language } \\ \text { ROC } & \text { Receiver Operating Characteristic } \\ \text { SoIVES } & \text { Social Values for Ecosystem Services } \\ \text { SOL } & \text { Structured Query Language } \\ \text { USGS } & \text { U.S. Geological Survey }\end{array}$





\title{
Social Values for Ecosystem Services, Version 4.0 (SolVES 4.0)_Documentation and User Manual
}

\author{
By Benson C. Sherrouse and Darius J. Semmens
}

\section{Abstract}

The geographic information system tool, Social Values for Ecosystem Services (SolVES), was developed to incorporate quantified and spatially explicit measures of social values into ecosystem service assessments. SolVES 4.0 provides an open-source version of SolVES, which was designed to assess, map, and quantify the social values of ecosystem services. Social values - the perceived, nonmarket values the public ascribes to ecosystem services, particularly cultural services, such as aesthetics and recreation — can be evaluated for various stakeholder groups. These groups are distinguishable by factors such as their attitudes and preferences regarding public uses (for example, motorized recreation and logging). As with previous versions, SolVES 4.0 derives a quantitative 10-point, social-values metric - the value index-from a combination of spatial and nonspatial responses to public value and preference surveys. The tool also calculates metrics characterizing the underlying environment, such as average distance to water and dominant landcover. SolVES 4.0 has been developed with Python using a QGIS user interface and a PostgreSQL database for required data. SolVES is integrated with Maxent maximum entropy modeling software to generate more complete social-value maps and offer robust statistical models describing the relation between the value index and explanatory environmental variables. A model's goodness of fit to a primary study area and its potential performance in transferring social values to similar areas using value-transfer methods can be evaluated. SolVES 4.0 provides an improved open-source, public-domain tool for decision makers and researchers to evaluate the social values of ecosystem services and to facilitate discussions among diverse stakeholders regarding the tradeoffs among ecosystem services in a variety of biophysical and social contexts including mountain, forest, coastal, riparian, agricultural, and urban environments around the globe.

\section{Introduction}

Social Values for Ecosystem Services, version 4.0 (SolVES 4.0), is a geographic information system (GIS) application developed by the U.S. Geological Survey (USGS) Geosciences and Environmental Change Science Center. SolVES incorporates quantified and spatially explicit social-values information, which can be collected through public value and preference surveys, into ecosystem service assessments. Social values are defined here as the perceived, nonmarket values the public ascribes to ecosystem services, particularly cultural services, such as aesthetics and recreation. SolVES was originally developed to address the need to account for differing values, attitudes, and preferences among diverse stakeholders in the analysis of tradeoffs among ecosystem services (Sherrouse and others, 2011, 2014, 2017). Ecosystem services provide tangible and intangible benefits to human life (Daily, 1997). Although these benefits may be quantified in monetary terms, they also are public goods that bypass the economy (Costanza and others, 1997). Furthermore, monetary values, even if available, are not always desirable because they can distract decision makers and stakeholders from the primary purpose of ecosystem management (Maleki, 2008). Decision makers require estimates of social values beyond those defined economically to assess the full range of ecosystem values. These social values involve sociocultural perceptions of human well-being that are derived from nature and measured using social assessments and other nonutilitarian means of capturing their value (Millennium Ecosystem Assessment, 2003; Cowling and others, 2008; Kumar and Kumar, 2008; Nijkamp and others, 2008). SolVES uses previous social-values mapping research (Brown and Reed, 2000; Brown and others, 2002, 2004; Reed and Brown, 2003; Brown, 2004; Brown and Alessa, 2005; Alessa and others, 2008) to implement a method for incorporating social values into the ecosystem service assessment process by quantifying and mapping these values across a study area as a "value index," which provides a spatial, nonmonetary metric statistically related to characteristics of the underlying physical environment.

SolVES 4.0 provides functionality to assess, map, and quantify social values such as aesthetics, biodiversity, and recreation by deriving social-value maps rendered as a 10-point 
value index (ranging from 0 to 10 ) from a combination of spatial and nonspatial responses to public value and preference surveys. SolVES 4.0 also calculates metrics characterizing the underlying environment, such as average distance to water and dominant land cover. Through its integration with Maxent maximum entropy modeling software (Phillips and others, 2004, 2006, 2017; Phillips and Dudík, 2008; Elith and others, 2010), SolVES 4.0 can generate more-complete socialvalue maps and offer statistical models describing the relation between the value index and explanatory environmental variables. Because of its flexible design, SolVES 4.0 users are able to define their own social values and public uses, model any number and types of environmental variables, select weighting options for mapped survey data, and modify the spatial resolution of analysis. SolVES 4.0 provides an improved open-source, public-domain tool for decision makers and researchers to evaluate the social values of ecosystem services and to facilitate discussions among diverse stakeholders regarding the tradeoffs among ecosystem services in a variety of biophysical and social contexts including mountain, forest, coastal, riparian, agricultural, and urban environments around the globe. SolVES 4.0 is available in a USGS software release (Sherrouse and Semmens, 2020).

\section{Maxent Maximum Entropy Modeling Software}

SolVES 4.0 is designed to run in conjunction with Maxent maximum entropy modeling software. Maxent was developed to model the geographic distribution of species; however, its modeling structure provides a framework that can be readily adapted to mapping the social values of ecosystem services. Maxent relies on point data representing observations of plant or animal species presence. Without true absence data (points where species are observed to be absent) available, Maxent generates randomly selected background points. Using these point data along with environmental variables that are judged to affect the suitability of the environment for a selected species, Maxent applies a machine-learning method to estimate a probability distribution of maximum entropy (closest to uniform) while satisfying constraints represented by the environmental variables. The logistic surfaces Maxent generates are most pertinent to its use with SolVES. Each cell contains a value from 0 to 1 , with higher values indicating locations more suitable as habitat for a species given the environmental conditions and the known presence of that species. In a social-values mapping context, the logistic output represents the relative intensity that survey respondents assign to a social-value type (analogous to a species) at a location given the underlying environmental characteristics and the respondents' identification of such locations as representing a particular social-value type. Together with the kernel density method already used by SolVES, Maxent's logistic output provides more complete maps within a study area where survey value and preference survey data are available.
Maxent also enhances SolVES functionality by generating statistical models describing the relation between mapped points and environmental variables (or features as operationalized by Maxent). Additionally, Maxent calculates Area Under the Curve (AUC) statistics for each model to evaluate its goodness of fit to the study area and the potential performance of the model in transferring social values to similar areas where primary survey data are not available. Maxent output includes jackknife statistics that can help SolVES users improve models by adjusting the environmental variables included in their analysis. Through an iterative process, SolVES users have the ability to repeat their selected analyses as different projects to generate models best suited for their purposes. More information regarding Maxent's AUC statistics is in the "Interpreting and Adjusting Maxent's Area Under the Curve Values and Variable Contributions" section in appendix 1.

This user manual does not attempt to provide an exhaustive description of Maxent. Its focus is instead on those features most pertinent to SolVES users. For additional information regarding Maxent, please refer to the Maxent website https://biodiversityinformatics.amnh.org/open_source/maxent/ that includes links to journal articles, tutorials, user groups, and software.

\section{New in SolVES 4.0}

SolVES 4.0 does not include any new functionality beyond what was previously available in SolVES 3.0; however, its release provides SolVES in an open-source environment. SolVES 4.0 was developed with Python and uses the free software packages QGIS for its user interface and PostgreSQL spatially enabled by PostGIS for its source database. Minor changes to the source database and user interface provide users with additional information about each socialvalue model and reduce the number of required user inputs. The Analyze Survey Data and Transfer Values tools are now directly accessible from the project setup screen. The addition of a table to the source database allows users to indicate that environmental data are either continuous or categorical, eliminating the need to identify the data type at runtime. Environmental data included in social-value models used for value transfer are automatically identified at runtime. The final map layout has been redesigned to increase the number of environmental data graphs that can be viewed and to provide the average nearest neighbor and maximum-value index statistics associated with each social-value model. An additional map background option using OpenStreetMap allows users to include additional reference information on their socialvalue maps.

\section{SolVES 4.0 Overview}

SolVES 4.0 was developed with Python as a custom plugin for QGIS open-source software. SolVES uses geospatial and tabular data as inputs to three separate models - the 
Ecosystem Services Social-Values Model, the Value Mapping Model, and the Value Transfer Mapping Model. The general process flow of these models is shown below (fig. 1).

The Ecosystem Services Social-Values Model and the Value Mapping Model operate together in a sequence as follows:

1. The user selects a public use and an attitude or preference regarding that use (for example, favor or strongly favor motorized recreation) to define a stakeholder group (survey subgroup). If a survey subgroup is not selected, SolVES will analyze all survey data.

2. Based on the user's selections, the Ecosystem Services Social-Values Model retrieves mapped points and socialvalue allocation amounts, if available, for the matching survey subgroup.

3. Using the allocation amounts the survey subgroup assigned to each social-value type as weights, the model calculates kernel density surfaces and average nearest neighbor statistics for the mapped points associated with every social-value type included in the survey.

4. By comparing kernel-density surfaces, the model identifies the highest rated social-value type and the location where it is most highly valued (maximum value in fig. 1).
5. The user starts the Value Mapping Model by selecting one or more social-value types (for example, aesthetics and recreation).

6. The model uses the maximum value to normalize the kernel density surface(s) of the selected social-value type(s) and then standardizes the calculated surface to produce a kernel density-based, value-index surface.

7. The model provides Maxent maximum entropy modeling software with the selected survey-point data along with user-selected environmental layers for the study area.

8. Maxent produces a map output based on the relation between the provided points and environmental layers and generates statistical model(s) (fig. 1) describing the relation.

9. The Value Mapping Model then derives a final socialvalue map from the Maxent map output in conjunction with data from the corresponding kernel-density, valueindex surfaces. Additionally, the Value Mapping Model calculates environmental metrics using the final socialvalue map. The map and associated metrics can then be compiled into a composite report.

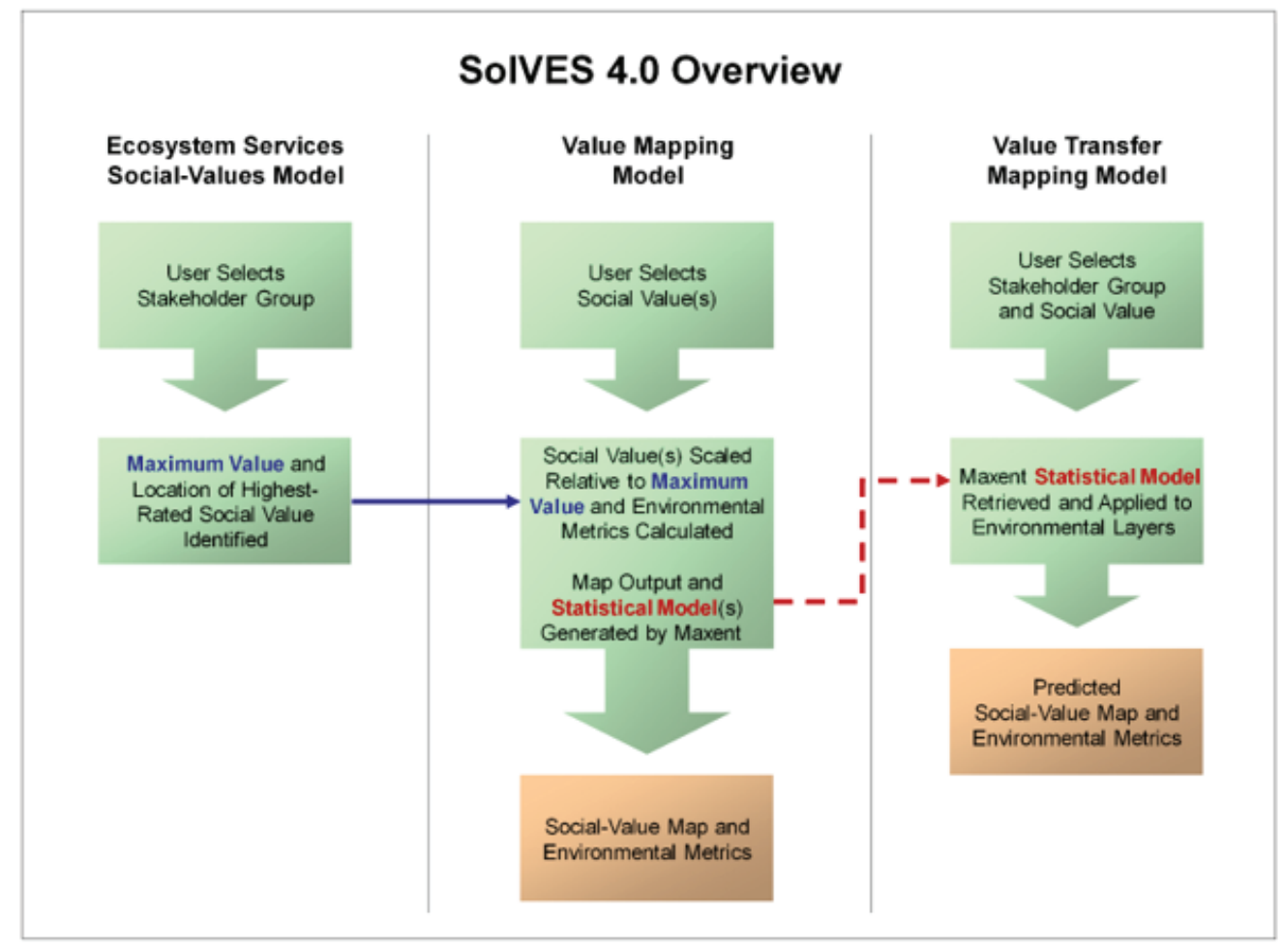

Figure 1. Generalized Social Values for Ecosystem Services, version 4.0, process flow. 
The Value Transfer Mapping Model operates independently of the other models but relies on their statistical model output. It operates as follows:

1. The user starts the Value Transfer Mapping Model and selects a stakeholder group (survey subgroup) and social-value type.

2. Based on the user's selections, the model retrieves the appropriate statistical model generated by Maxent.

3. Maxent applies the retrieved statistical model to the environmental layers for the receiving study area.

4. The model standardizes the Maxent output to generate a predicted social-value map and calculates environmental metrics using the predicted social-value map. The map and associated metrics can then be compiled into a composite report.

\section{Hardware and Software Requirements}

SolVES 4.0 was developed and tested on systems running Microsoft Windows 10 Enterprise Edition using a 64-bit processor. The software listed below are required to run SolVES 4.0. Installation files for the required software are included in the full SolVES 4.0 download. Additionally, either Java Runtime or Amazon Corretto must be available on the system running SolVES 4.0 for it to access and run Maxent 3.4.1.

- QGIS 3.8.2

- PostgreSQL 11.7

- PostGIS 2.5.3

- Maxent 3.4.1

SolVES 4.0 processing times will vary depending on factors such as system memory and processor speed, the spatial resolution and extent of the study area, and the number of variables selected for analysis.

\section{Navigating the User Manual}

After completing the software and sample data installation procedures outlined in the "Installation" section, users can choose to proceed directly to the "Project Setup," "Analyze Survey Data," "Transfer Values," and "View Results" sections, treating those sections of the user manual as a brief tutorial for gaining hands-on experience using SolVES 4.0. Alternatively, users can continue through the entire document for additional information about SolVES 4.0. Those planning to use SolVES 4.0 to analyze their own data should, before proceeding, carefully review the "Data Requirements," "Directory Structure," and "Data Management" sections of appendix 1; and the "Preparing Data for the 'solves' Database" and "Loading Data to the 'solves' Database" sections in appendix 2. Please note that any reference to "solves" in this user manual refers specifically to the source database, and "SolVES" refers to the SolVES 4.0 application or directory structure.

\section{Installation}

The following sections assume that the required software have not been previously installed. Most of the described installations require administrator-level privileges and might require users to consult with their own information technology support staff if operating in an enterprise environment. Those already using QGIS and PostgreSQL might wish to determine the versions they have installed and proceed to the "QGIS Settings for SolVES 4.0" section and subsequent sections to determine if their current installations support SolVES 4.0; however, no testing has occurred beyond the specific software versions listed in the "Hardware and Software Requirements" section. At several points during the installation, users will be required to accept various open-source licensing agreements. If users have any questions or concerns regarding these licenses, users should consult with their own information technology support staff.

\section{SoIVES 4.0 Download}

1. Download the "SolVES_V4.zip" file from the SolVES website, http://solves.cr.usgs.gov (or alternatively, https://www.usgs.gov/centers/gecsc/science/socialvalues-ecosystem-services-solves?qt-science_center objects $=0 \#$ qt-science_center_objects), by clicking on the "SolVES_4.0_Tool” link.

2. Unzip the file to the desired location and note the full path of the SolVES directory (for example, C:ISolVES). The location and contents of the SolVES top-level directory should appear as shown in figure 2. All installation files are within the \SolVES Install folder.

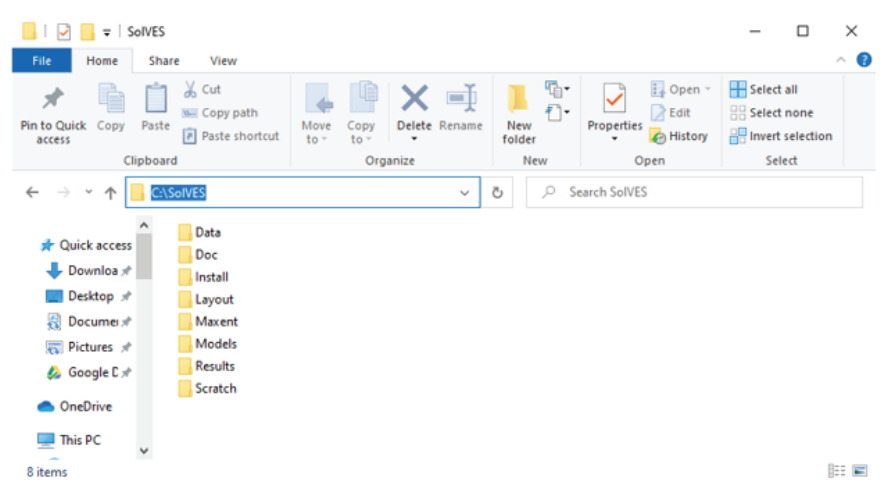

Figure 2. SolVES top-level directory structure after unzipping the "SolVES_V4.zip" file. 


\section{PostgreS0L Installation}

Complete the following steps if PostgreSQL 11.7 is not already installed:

1. Navigate to the $\backslash$ SolVES $\backslash$ Install $\backslash P o s t g r e S Q L$ folder in the SolVES directory (fig. 3).

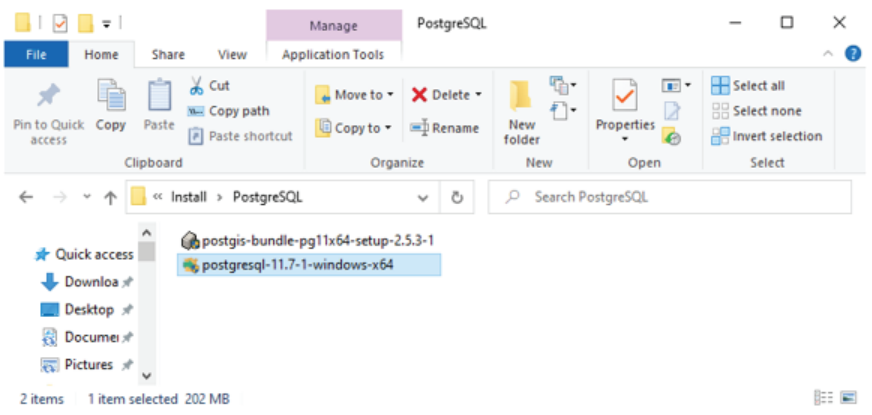

Figure 3. Location of the PostgreSQL installation file.

2. Select and run the "postgresql-11.7-1-windows-x64.exe" installation file, wait for the setup screen to appear, and select "Next" (fig. 4).

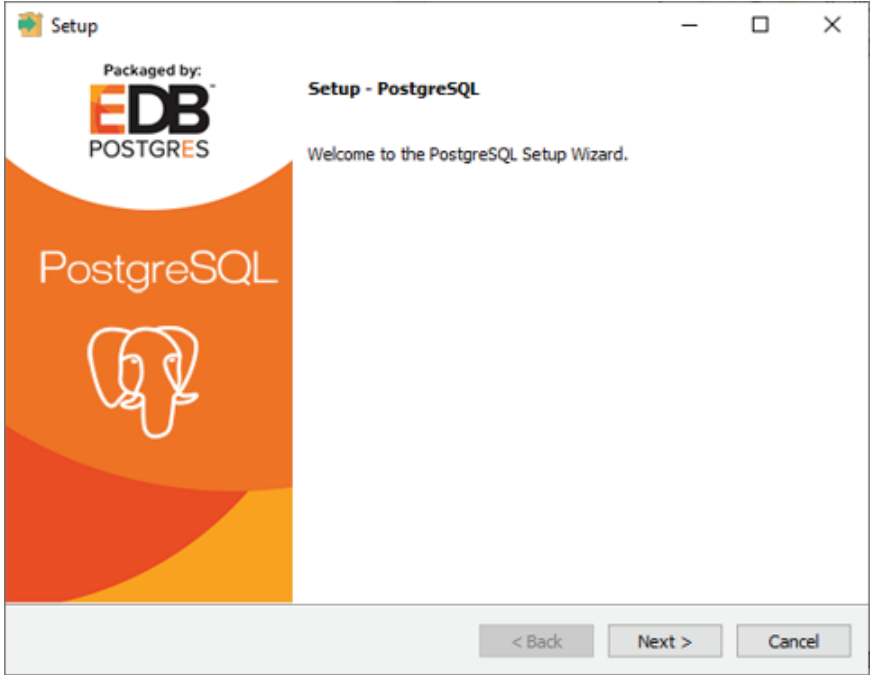

Figure 4. PostgreSOL setup screen.
3. Confirm the installation directory location and select "Next" (fig. 5).

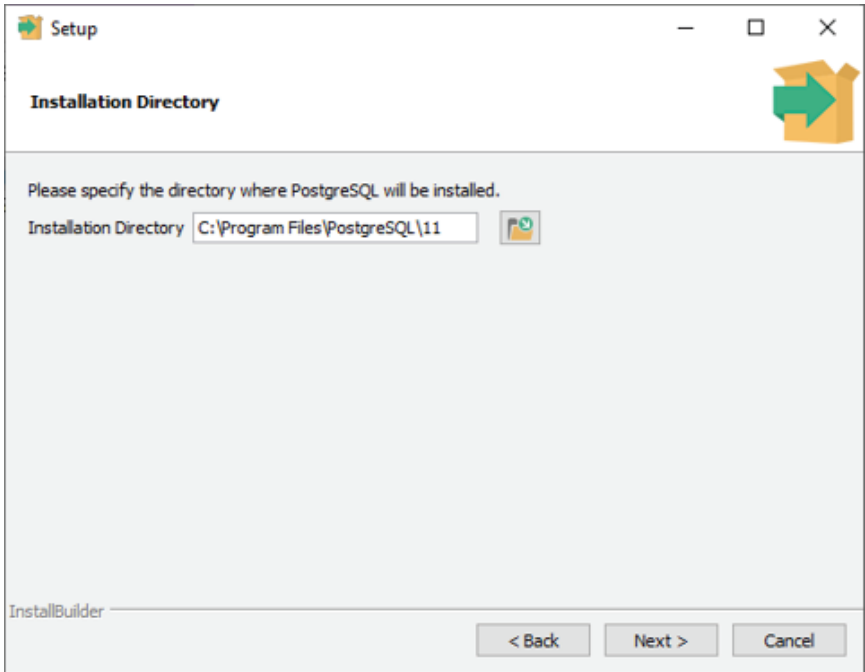

Figure 5. PostgreSOL 11.7 installation directory screen.

4. Confirm all components are selected and select "Next" (fig. 6).

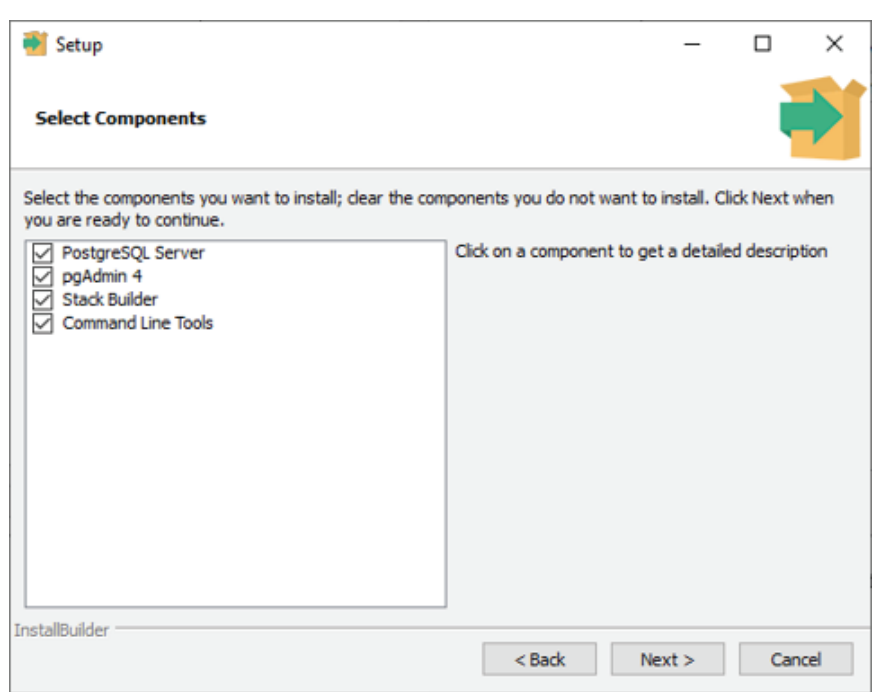

Figure 6. PostgreSOL 11.7 select components screen. 
5. Confirm the data directory location and select "Next" (fig. 7).

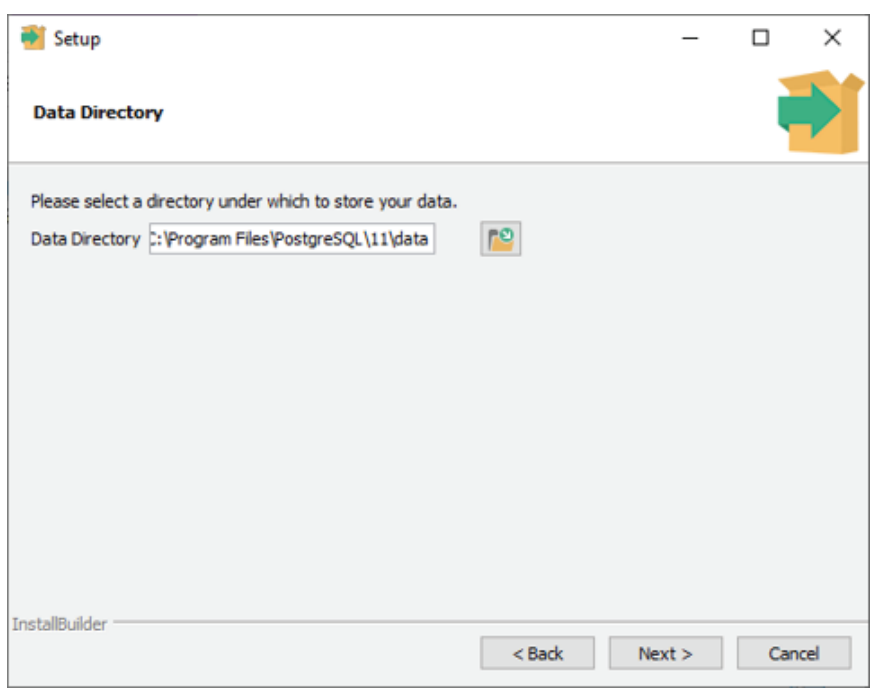

Figure 7. PostgreSQL 11.7 data directory screen.

6. Enter "postgres" as the password and select "Next" (fig. 8).

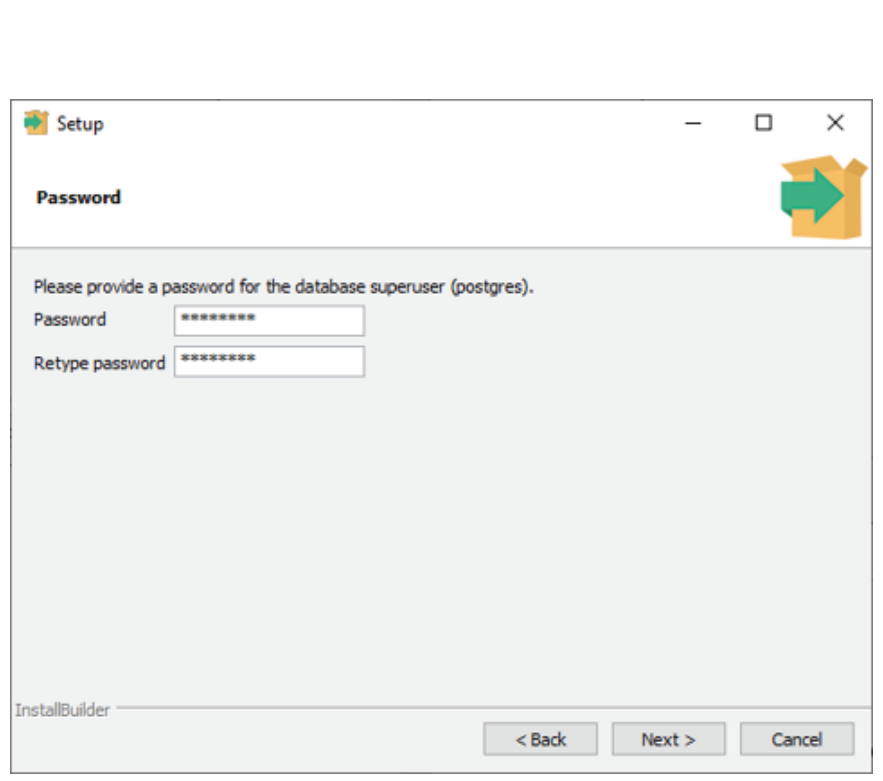

Figure 8. PostgreSOL 11.7 password screen.
7. Select "Next" to accept port "5432" (fig. 9).

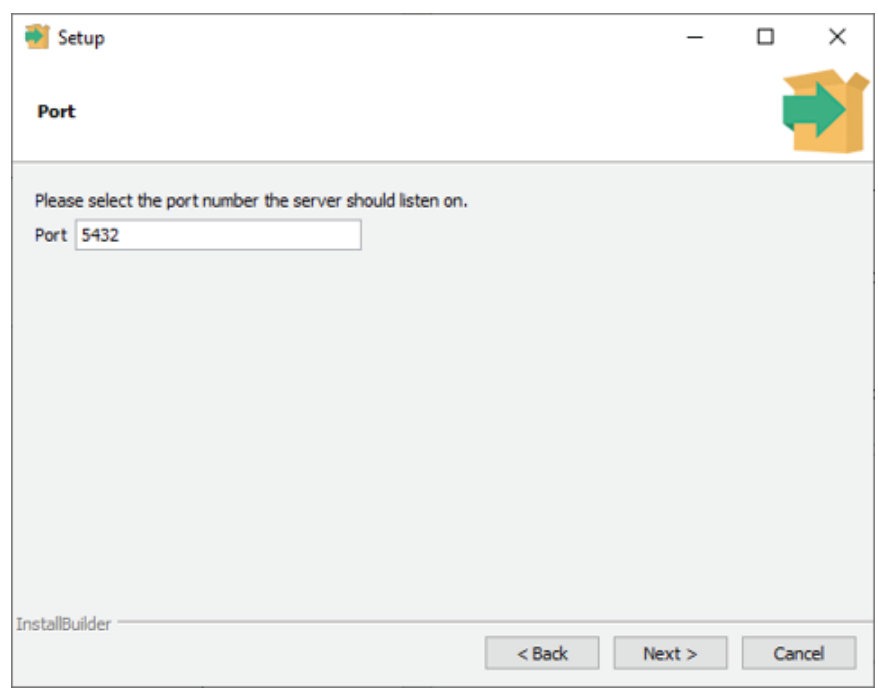

Figure 9. PostgreSOL 11.7 port screen.

8. Select "Next" to accept "[Default locale]" (fig. 10).

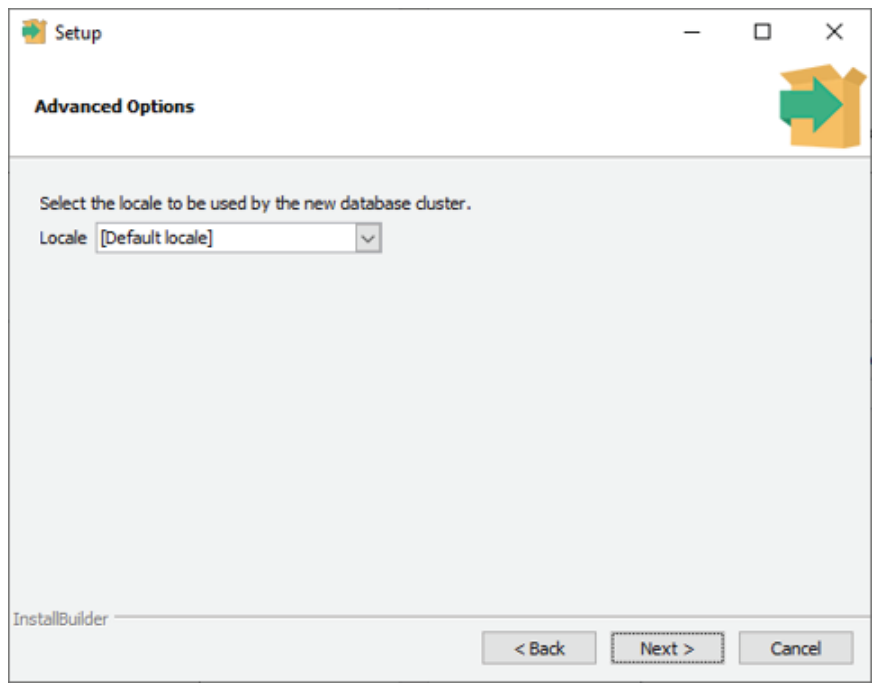

Figure 10. PostgreSOL 11.7 advanced options screen. 
9. Select "Next" on the pre-installation summary screen (fig. 11).

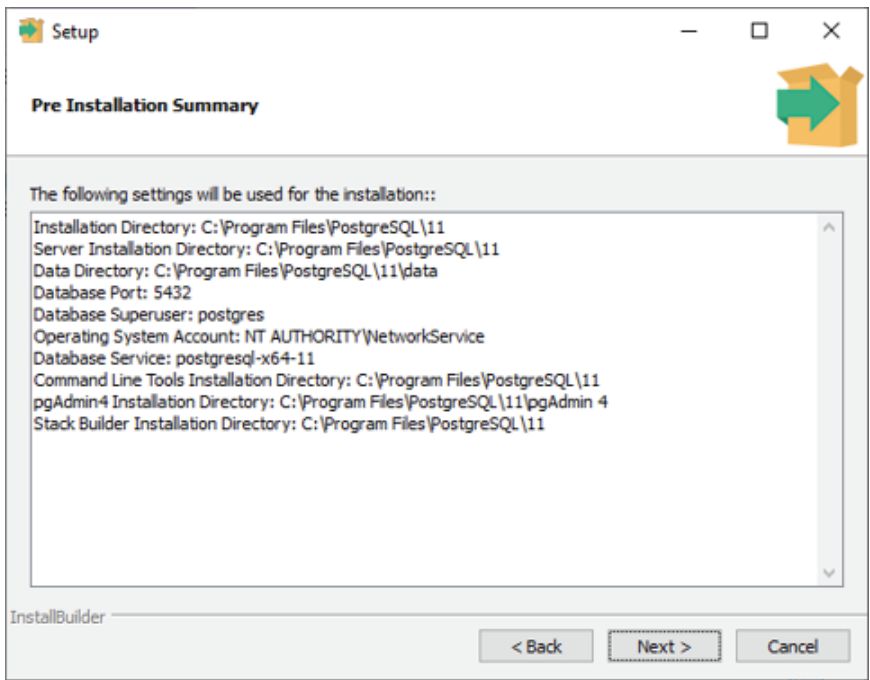

Figure 11. PostgreSQL 11.7 pre-installation summary screen.

10. Select "Next" on the ready to install screen (fig. 12) to begin installation (fig. 13).

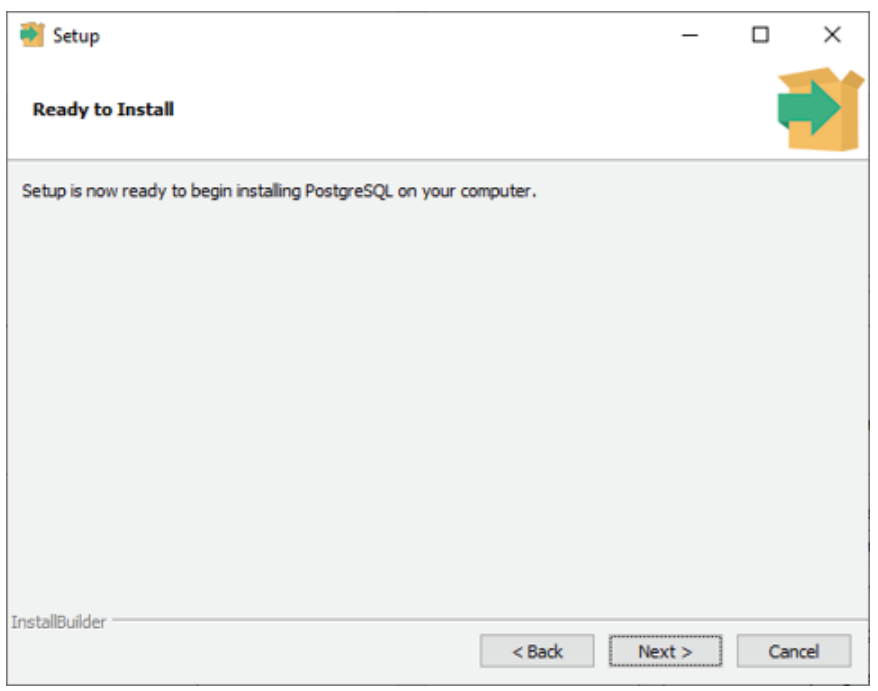

Figure 12. PostgreSOL 11.7 ready to install screen.

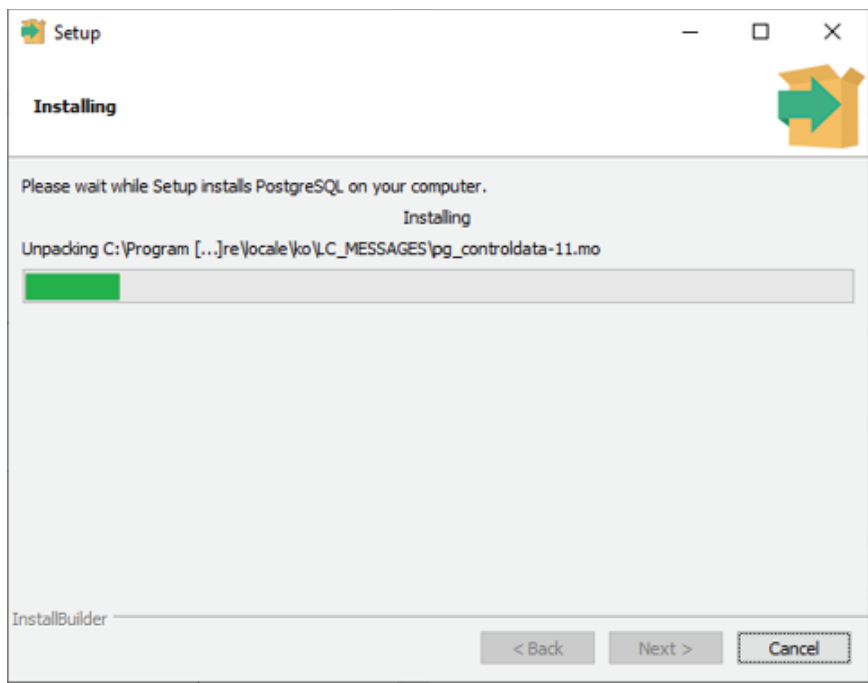

Figure 13. PostgreSOL 11.7 installation in progress.

11. Confirm checkbox is selected and select "Finish" (fig. 14).

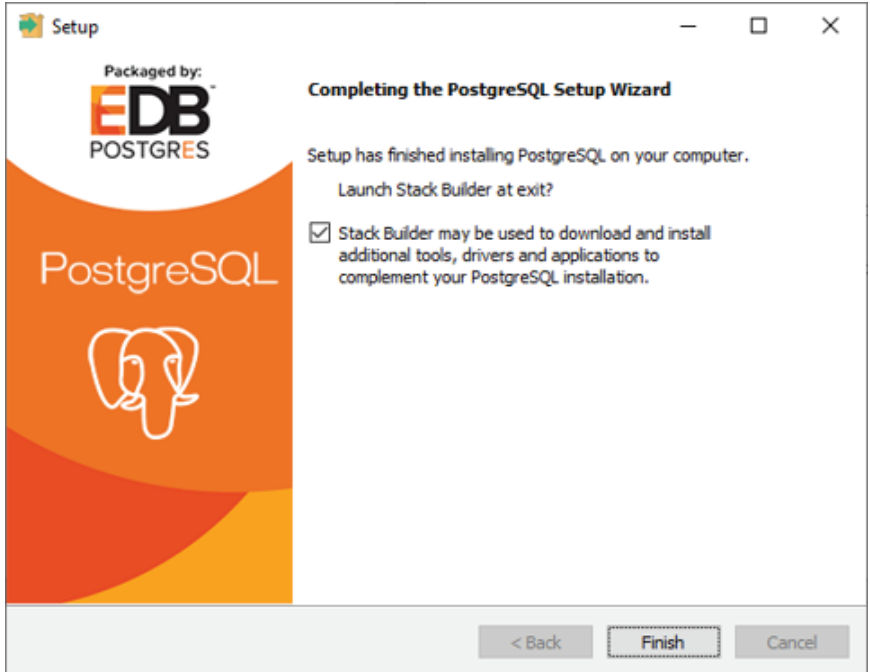

Figure 14. Completing the PostgreSOL 11.7 setup wizard screen.

At this point, PostgreSQL has been installed. The following steps continue the installation process using Stack Builder 4.1.0 to install PostGIS 2.5.3, an extension that enables the spatial capabilities of PostgreSQL databases. If errors are encountered when Stack Builder starts, refer to the "Unable to Install PostGIS" subsection of the "Troubleshooting" section; otherwise continue to step 12 . 
12. After the Stack Builder welcome screen appears, select "PostgreSQL 11 (x64) on port 5432" from the dropdown menu and select "Next" (fig. 15).

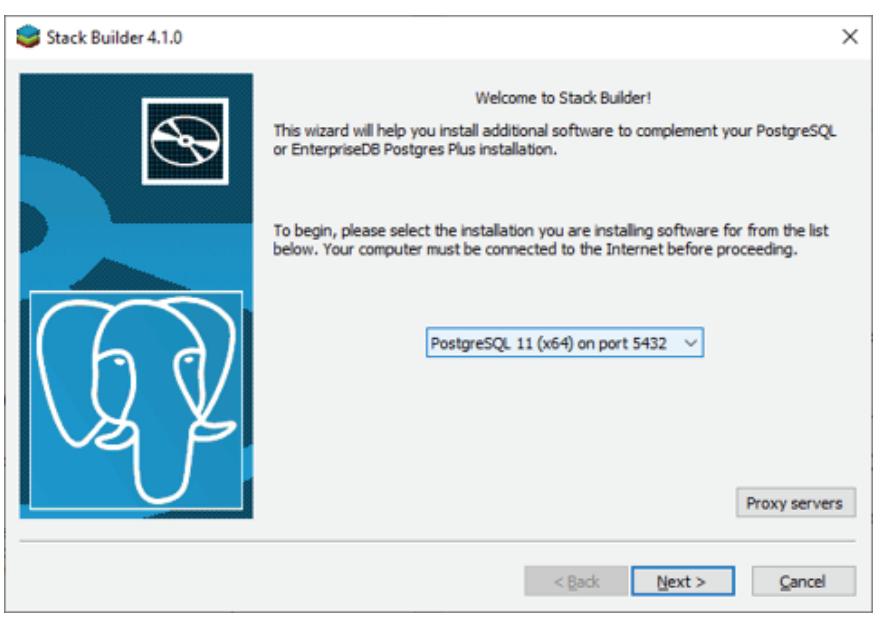

Figure 15. Stack Builder 4.1.0 welcome screen.

13. Check the box for "PostGIS 2.5 Bundle for PostgreSQL11 (64 bit) v2.5.3" under "Spatial Extensions" and select "Next" (fig. 16).

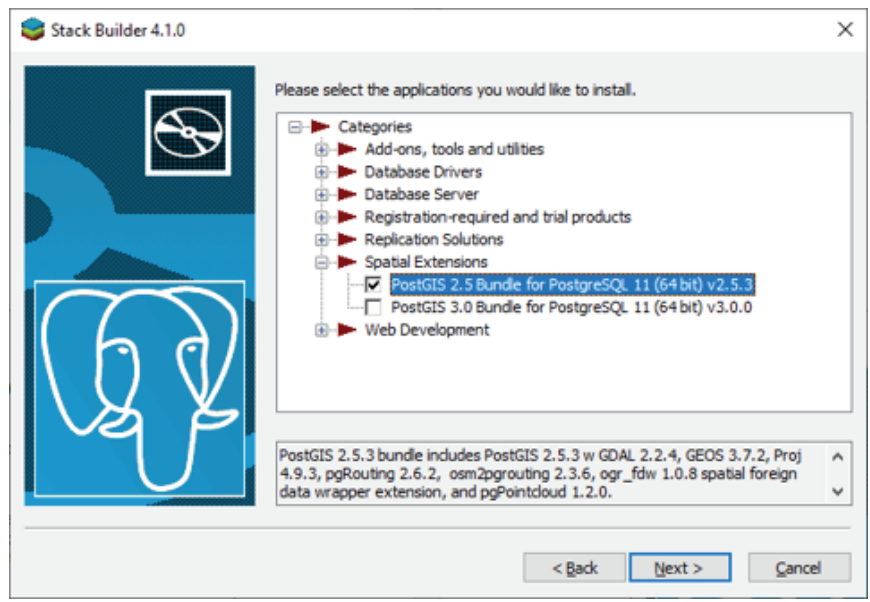

Figure 16. PostGIS 2.5.3 selection screen of Stack Builder 4.1.0.
14. Confirm that PostGIS 2.5.3 is included in the list of selected packages and select "Next" (fig. 17).

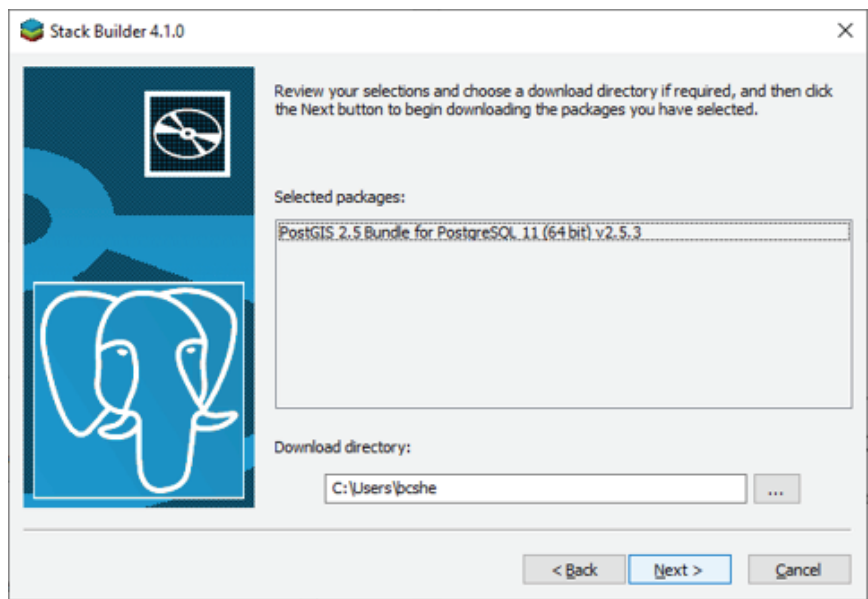

Figure 17. Selected packages screen of Stack Builder 4.1.0.

15. Select "I Agree" to the license agreement (fig. 18).

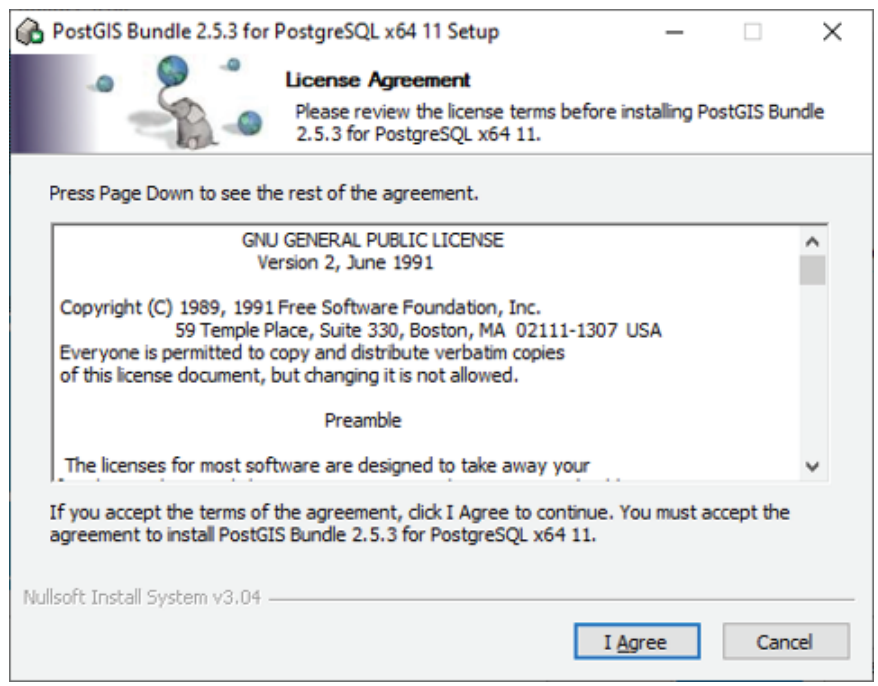

Figure 18. License agreement screen for PostGIS 2.5.3. 
16. Confirm the "Skip Installation" checkbox is unchecked and select "Next" (fig. 19).

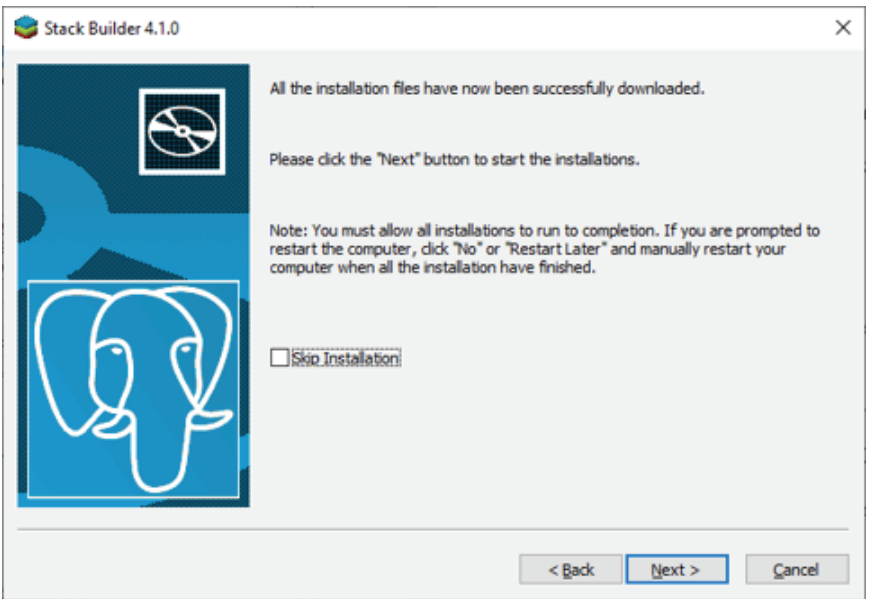

Figure 19. Confirm PostGIS 2.5.3 installation screen of Stack Builder 4.1.0.

17. Confirm that only the "PostGIS" checkbox is checked and select "Next" (fig. 20).

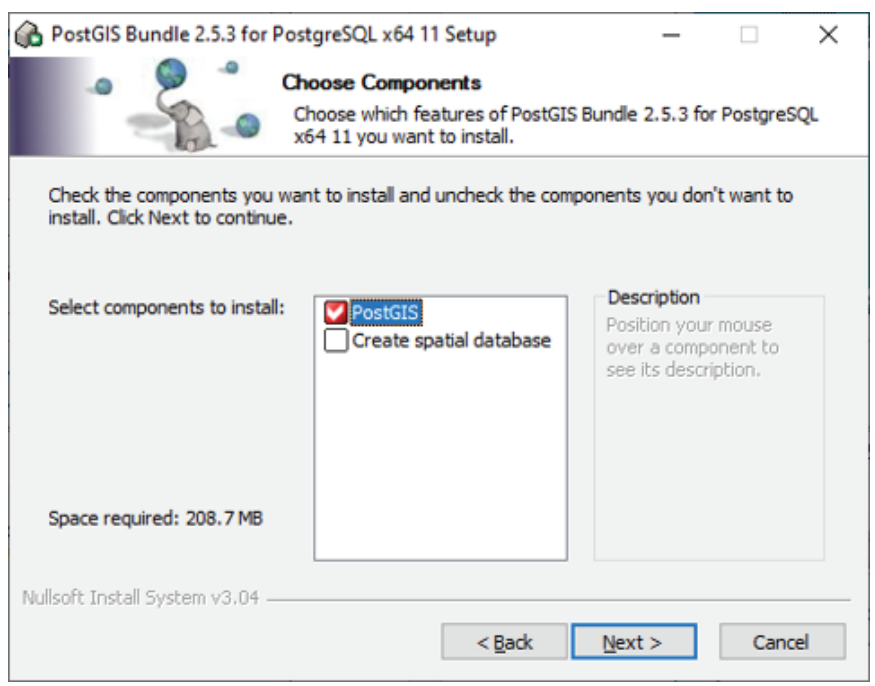

Figure 20. PostGIS 2.5.3 choose components screen.
18. Select "Next" to confirm the destination folder (fig. 21) and begin installation (fig. 22).

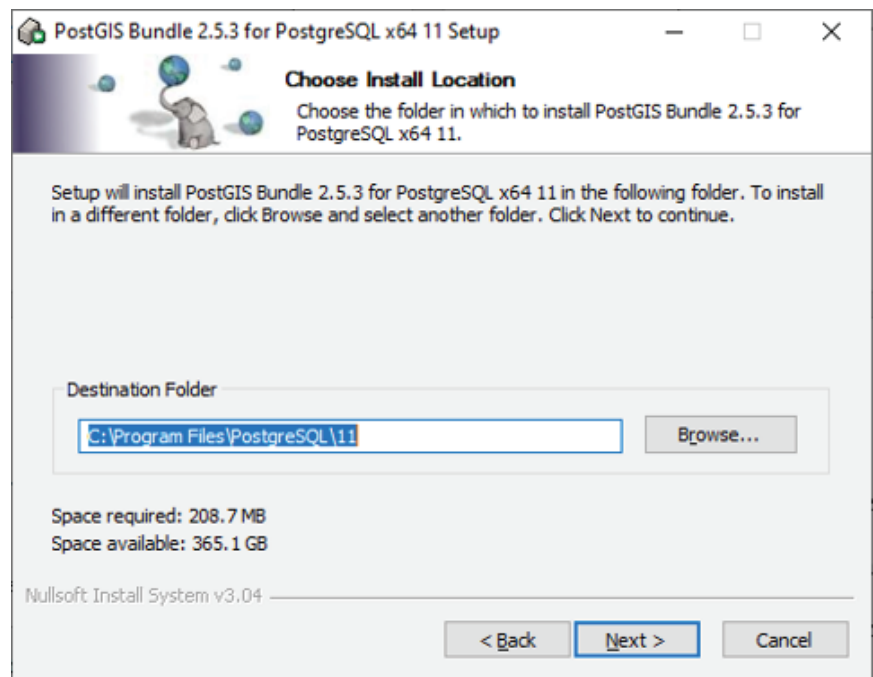

Figure 21. PostGIS 2.5.3 installation location screen.

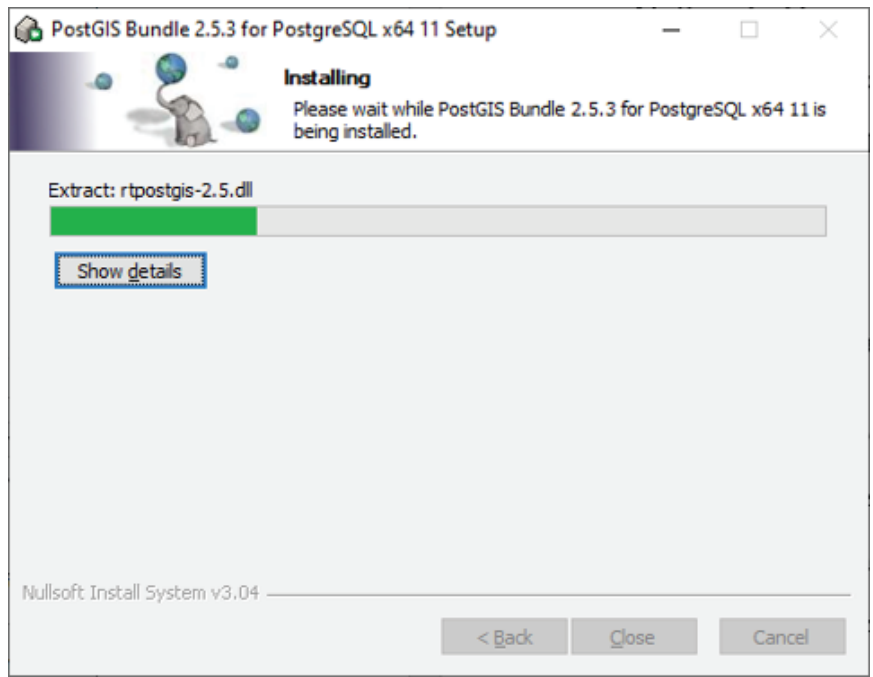

Figure 22. PostGIS 2.5.3 installation in progress. 
19. The next three screens ask for user input regarding the registration of environment variables and the enabling of drivers (fig. 23). Select "Yes" on all screens.

\section{PostGIS Bundle 2.5.3 for PostgreSQL x64 11 Setup}

Would you like us to register the GDAL_DATA environment variable for you, needed for raster transformation to work properly? This will overwrite existing settings if you have them.

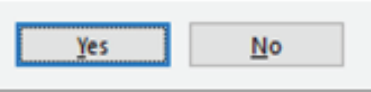

PostGIS Bundle 2.5.3 for PostgreSQL $\times 6411$ Setup

Raster drivers are disabled by default. To change you need to set POSTGIS_GDAL_ENABLED_DRIVERS environment variable and will need to restart your PostgreSQL service for changes to take effect. Set POSTGIS_ENABLED_DRIVERS to common drivers GTiff, PNG, JPEG, XYZ, DTED, USGSDEM, AAIGrid?

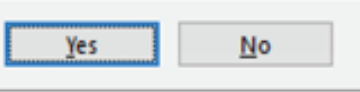

PostGIS Bundle 2.5.3 for PostgreSQL $\times 6411$ Setup

Raster out of $d b$ is disabled by default. To enable POSTGIS_ENABLE_OUTDB_RASTERS environment variable needs to be set to 1 . Enable out db rasters?

Figure 23. PostGIS 2.5.3 environmental variable and driver enabling message screens.
20. Select "Close" on the installation complete screen (fig. 24).

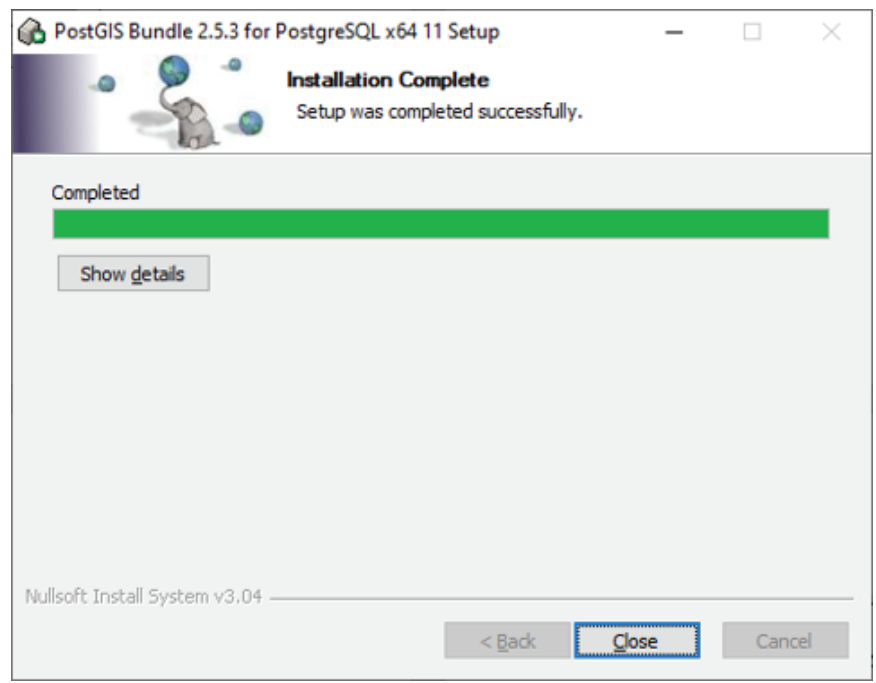

Figure 24. PostGIS 2.5.3 installation complete screen.

21. Select "Finish" on the installation completed screen (fig. 25).

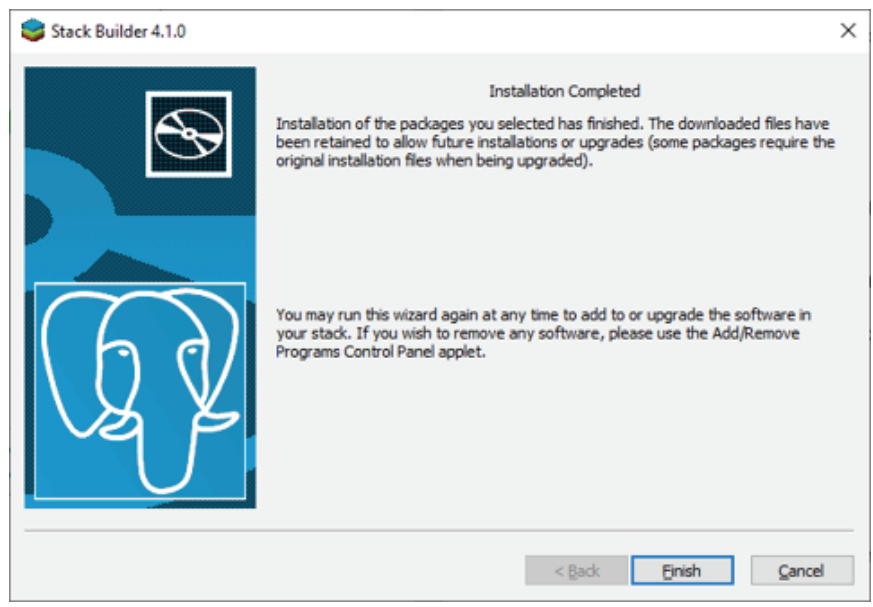

Figure 25. PostGIS 2.5.3 installation completed screen of Stack Builder 4.1.0.

At this point, PostgreSQL has been installed and spatially enabled with PostGIS. Proceed to the "QGIS Installation" section. 


\section{QGIS Installation} installed:

Complete the following steps if QGIS is not already

1. Navigate to the $\backslash$ SolVES $\backslash$ Install $\backslash Q G I S$ folder in the SolVES directory (fig. 26).

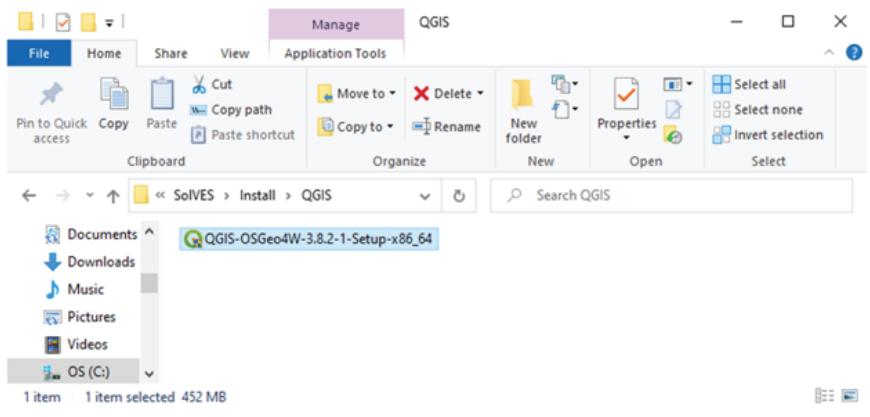

Figure 26. Location of the QGIS 3.8.2 installation file.

2. Double-click the installation file and wait for the setup wizard screen to appear and select "Next" (fig. 27).

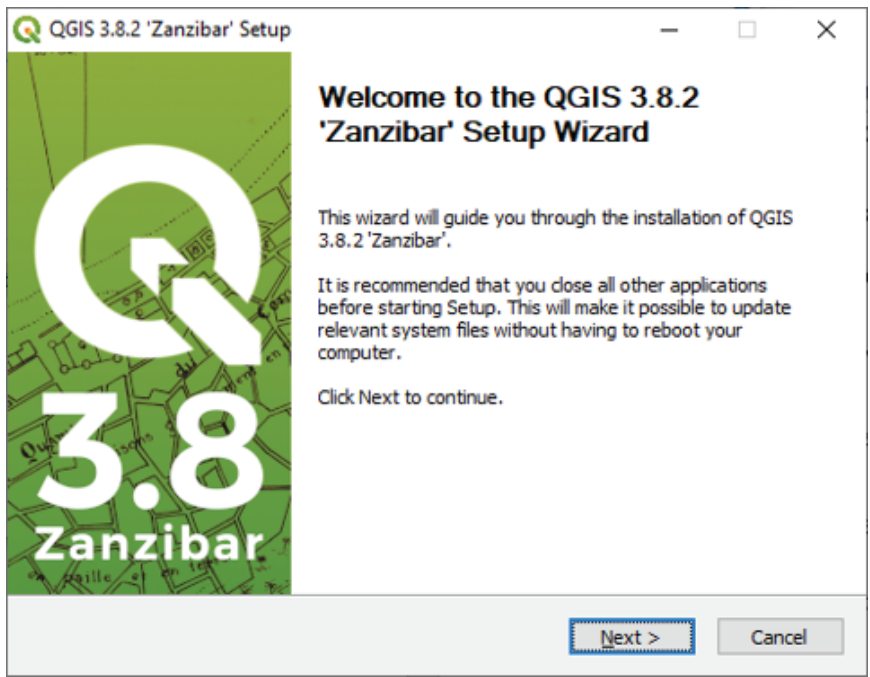

Figure 27. OGIS 3.8.2 setup wizard screen.
3. Select "I Agree" on the license agreement screen (fig. 28).

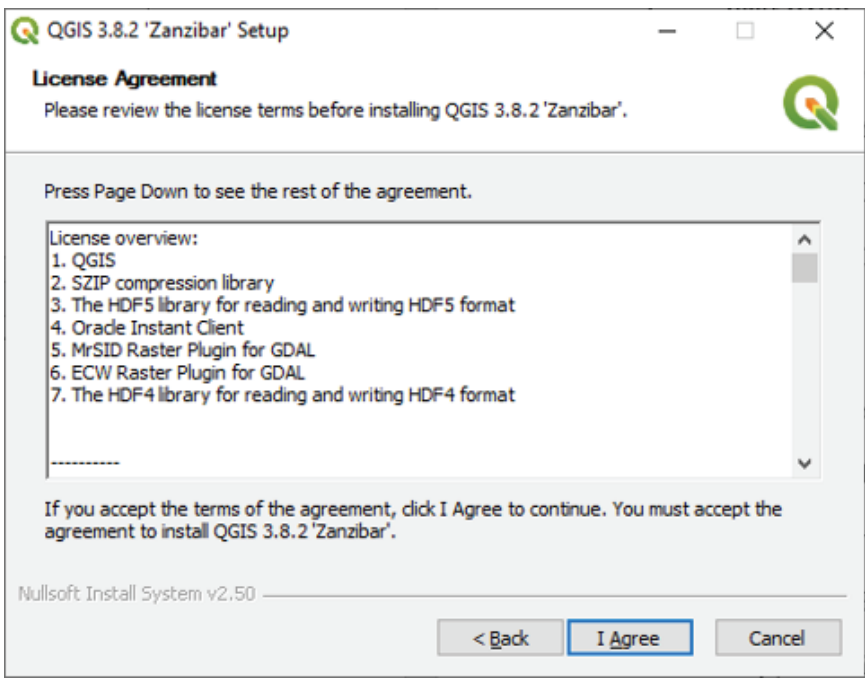

Figure 28. OGIS 3.8.2 license agreement screen.

4. Confirm the install location and select "Next" (fig. 29).

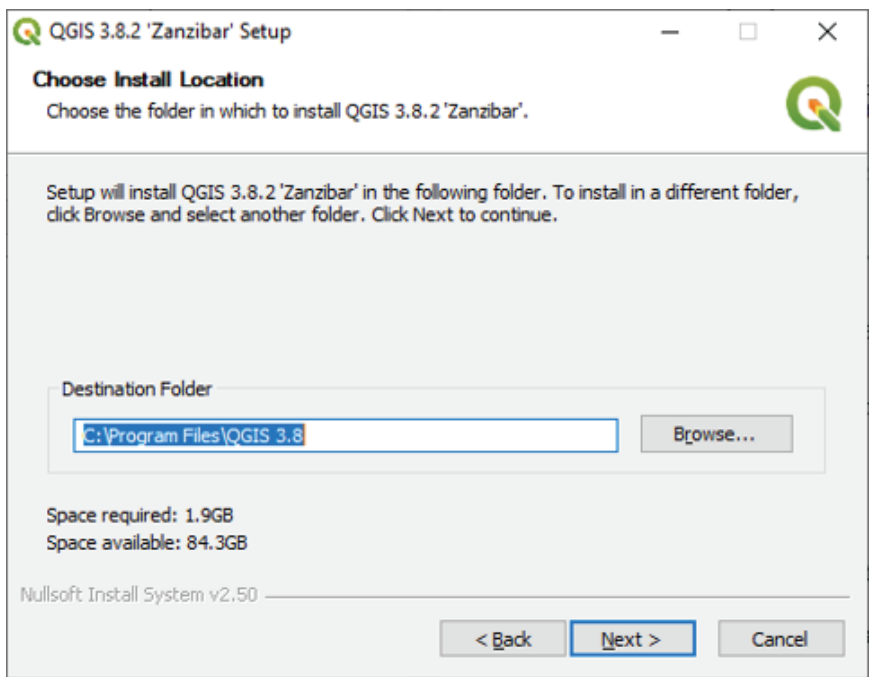

Figure 29. QGIS 3.8.2 choose install location screen. 
5. Confirm that only the QGIS checkbox is checked and select "Install" (fig. 30) to begin installation (fig. 31).

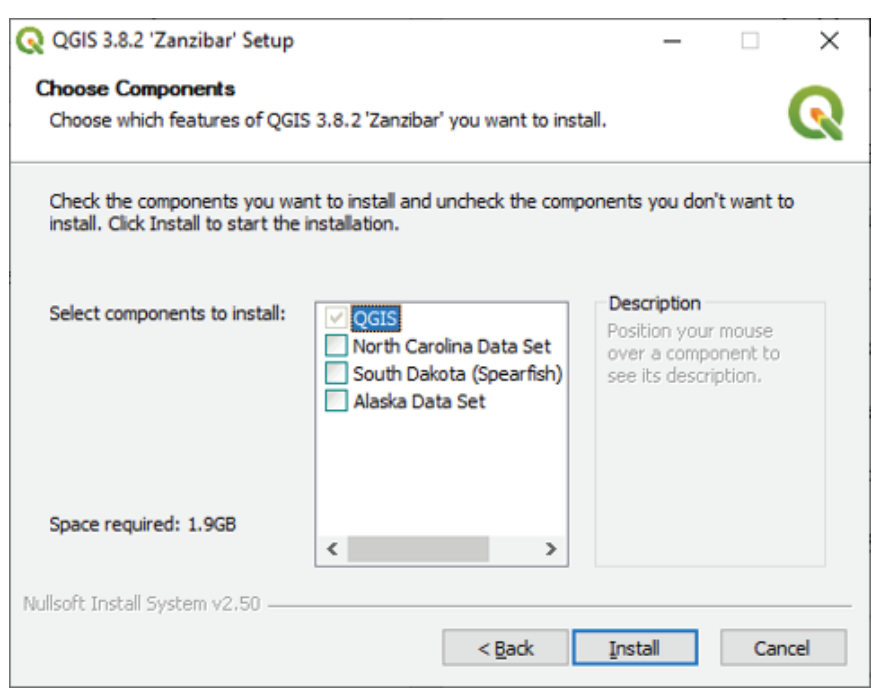

Figure 30. OGIS 3.8.2 choose components screen.

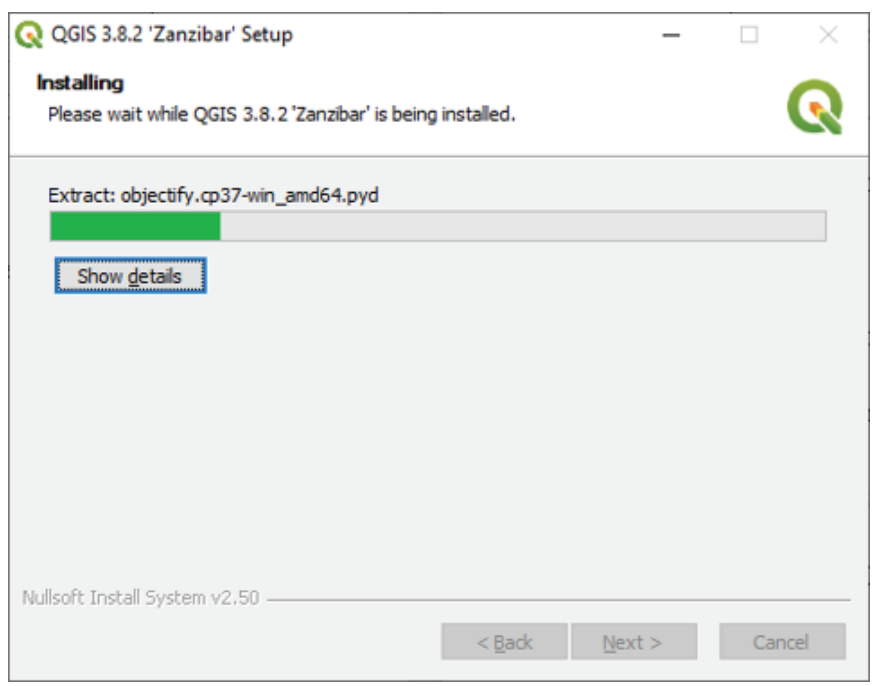

Figure 31. OGIS 3.8.2 installation in progress.
6. Select "Finish" on the setup completion screen (fig. 32).

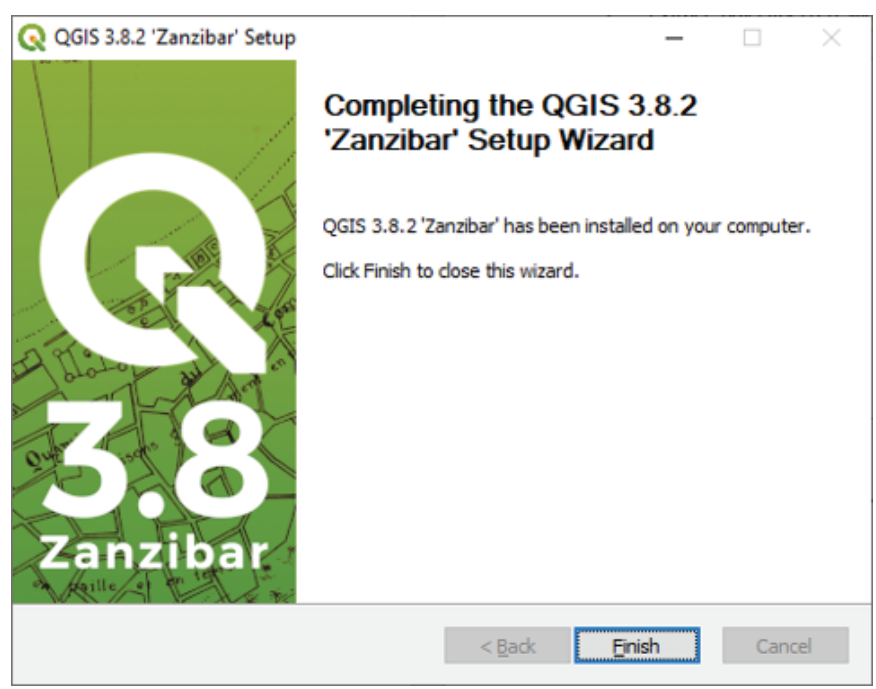

Figure 32. QGIS 3.8.2 setup completion screen.

At this point, QGIS has been installed. Continue to the "QGIS Settings for SolVES 4.0" section. 


\section{QGIS Settings for SoIVES 4.0}

The following steps are necessary to confirm that all QGIS settings are adjusted to properly install the SolVES 4.0 plugin:

1. Start QGIS. The first time QGIS is started after installation, a welcome message will appear. Select "Let's get started!" (fig. 33).

Q Welcome to QGIS

Welcome to QGIS 3.8.2-Zanzibar

Check out the change log for all the new stuff.

Figure 33. QGIS 3.8.2 welcome screen.

2. Select Settings $>$ Options $>$ System from the QGIS main menu.
3. Confirm that the "PATH" environment variable includes the path to your Java Runtime or Amazon Corretto directory (for example, C: $\backslash$ Program Files (x86) \Java jre1.8.0_241 \bin or C: Program Files $\backslash$ Amazon Corretto $\backslash$ jdk11.0.4_10〉bin) (fig. 34).

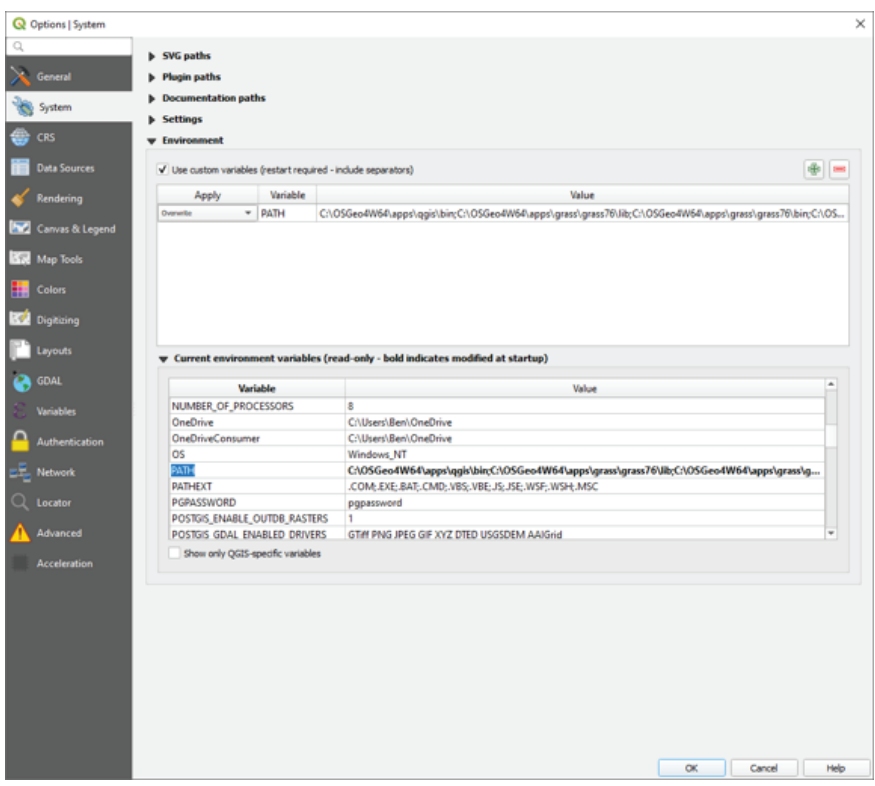

Figure 34. Modifying QGIS 3.8.2 environment variables.

4. If the directory is not included, check the "Use custom variables (restart required-include separators)" checkbox and select the green plus icon.

5. Select "Overwrite" from the "Apply" drop-down box.

6. Type "PATH" in the variable field.

7. Copy the existing values from the "PATH" variable in the current environmental variables and paste into the value field of the "PATH" custom variable.

8. Add a semicolon at the end of the pasted values, and then copy and paste the Java Runtime or Amazon Corretto directory path after the semicolon.

9. Select "OK" and restart QGIS. 
10. Select Settings $>$ Options $>$ Processing from the QGIS main menu.

11. Select "Do not filter (better performance)" from the "Invalid features filtering" drop-down and select "OK" (fig. 35).

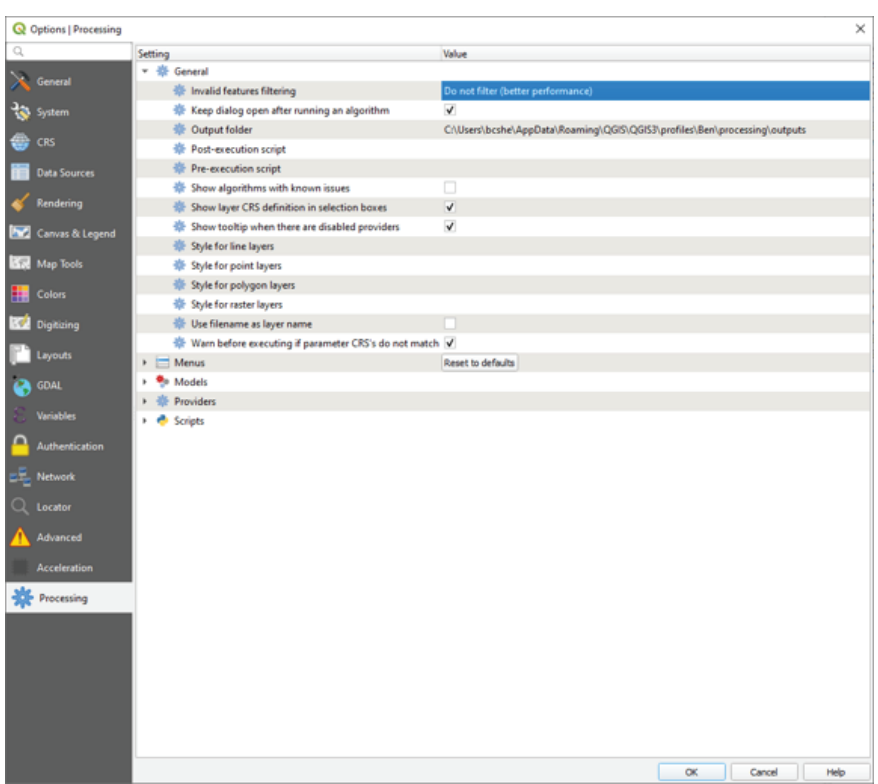

Figure 35. Setting the invalid features filtering value in QGIS 3.8.2.

At this point, QGIS is ready to install and run the SolVES 4.0 plugin. Continue to the "Installing the SolVES 4.0 Plugin" section.

\section{Installing the SolVES 4.0 Plugin}

1. Select Plugins $>$ Manage and Install Plugins... $>$ Install from ZIP from the QGIS main menu (fig. 36).

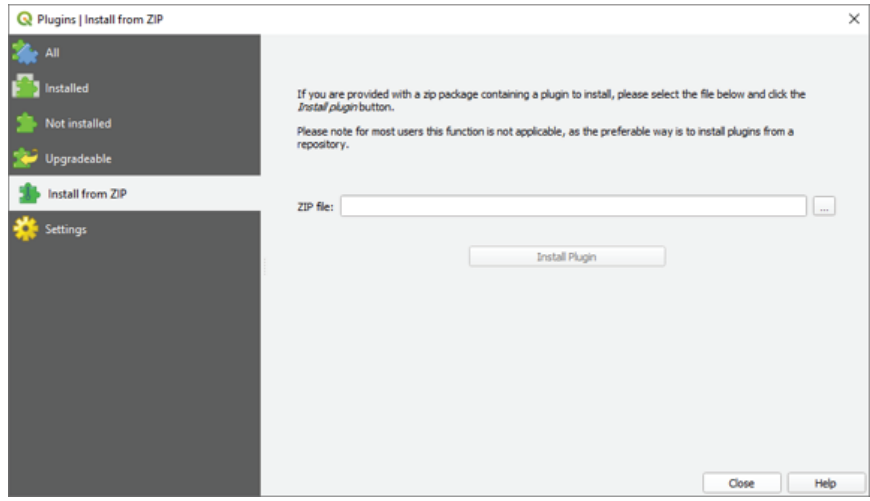

Figure 36. OGIS 3.8.2 plugins install from ZIP screen.

2. Navigate to the \SolVES \nstall $\backslash$ directory and select the "solves.zip" installation file (fig. 37).

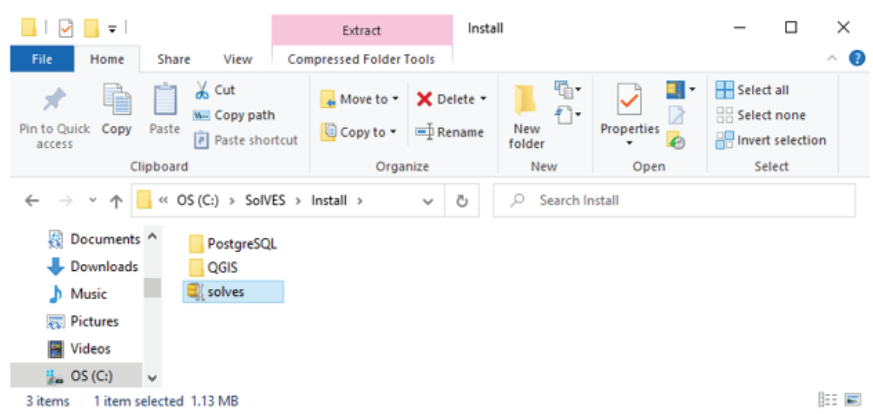

Figure 37. Location of the "solves.zip" plugin installation file. 
3. Select "Install Plugin" (fig. 38) to complete SolVES plugin installation.

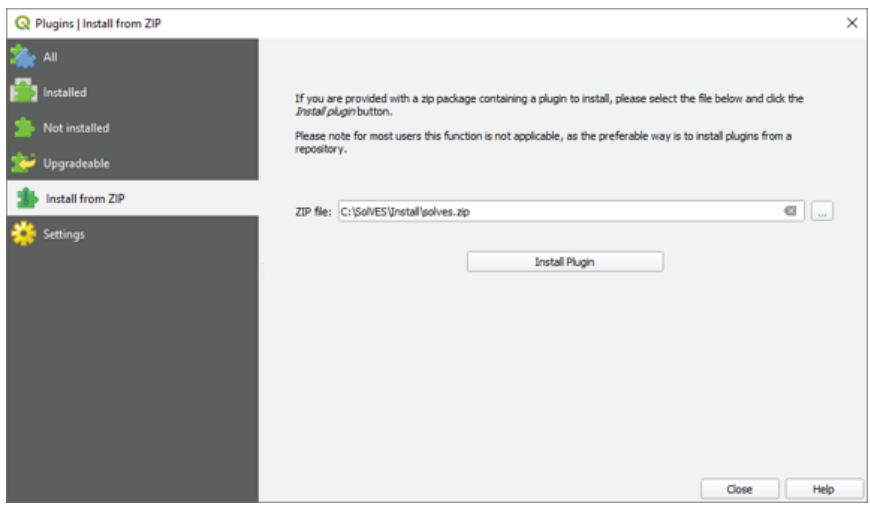

Figure 38. Install plugin selected "solves.zip" file in QGIS 3.8.2.

4. A warning message will appear regarding installing plugins from ZIP files (fig. 39). Select "Yes" to continue. The "Don't show this again" checkbox can be selected to avoid this message in the future if desired.

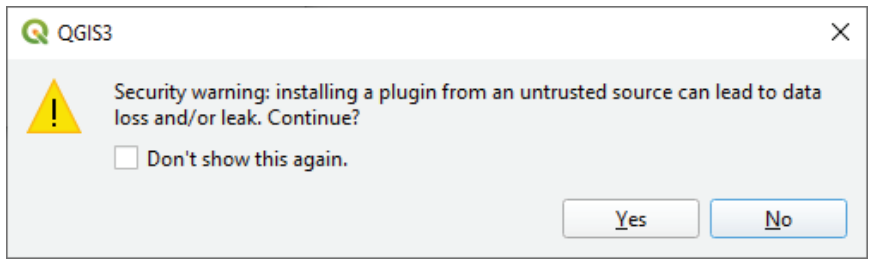

Figure 39. Plugin installation warning screen of QGIS 3.8.2.

5. A "Plugin installed successfully" message will appear briefly (fig. 40). Select "Close."

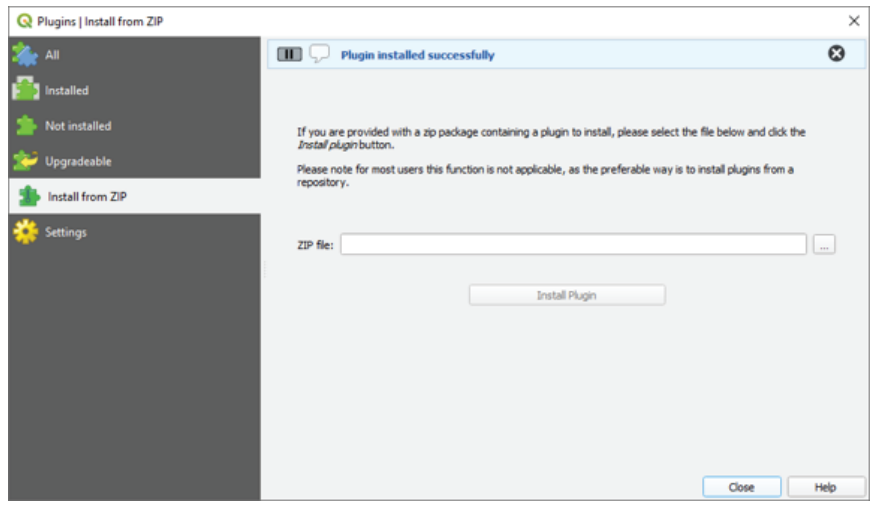

Figure 40. Successful installation message of QGIS 3.8.2.
6. The SolVES plugin should now be visible under the plugins menu (fig. 41).

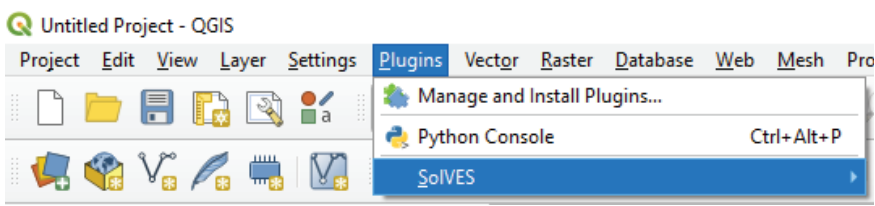

Figure 41. Successfully installed SolVES plugin.

7. If the SolVES plugin is not visible, select Plugins $>$ Manage and Install Plugins... from the QGIS main menu, select "Installed" from the QIS plugins menu, select the checkbox next to "SolVES," and then select "Close" (fig. 42). installed.

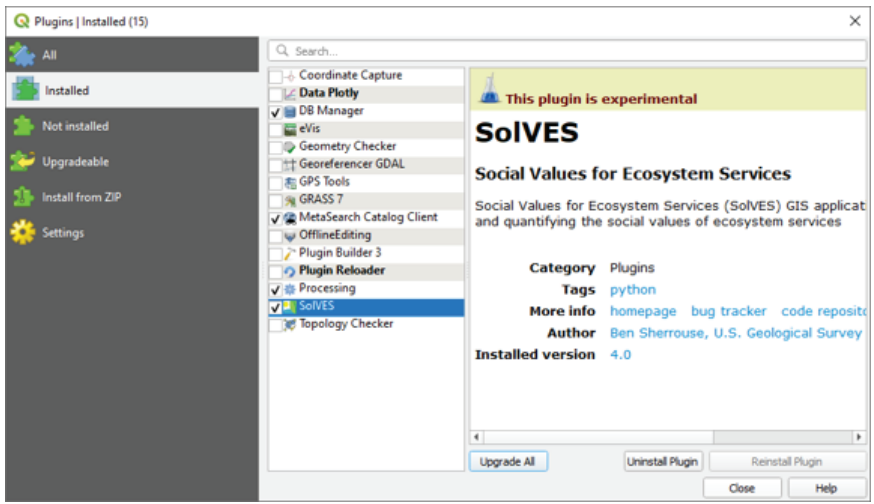

Figure 42. Selecting visibility of SoIVES plugin in QGIS 3.8.2.

At this point, the SolVES 4.0 QGIS plugin has been 


\section{Confirming Windows Regional Settings}

SolVES users outside the United States should access the Windows Control Panel region and language settings on their computer to confirm these settings are compatible with SolVES. This can be accomplished by following the steps outlined below:

1. Enter "control panel" in the Windows search tool located on the taskbar and then select the Control Panel app (fig. 43).

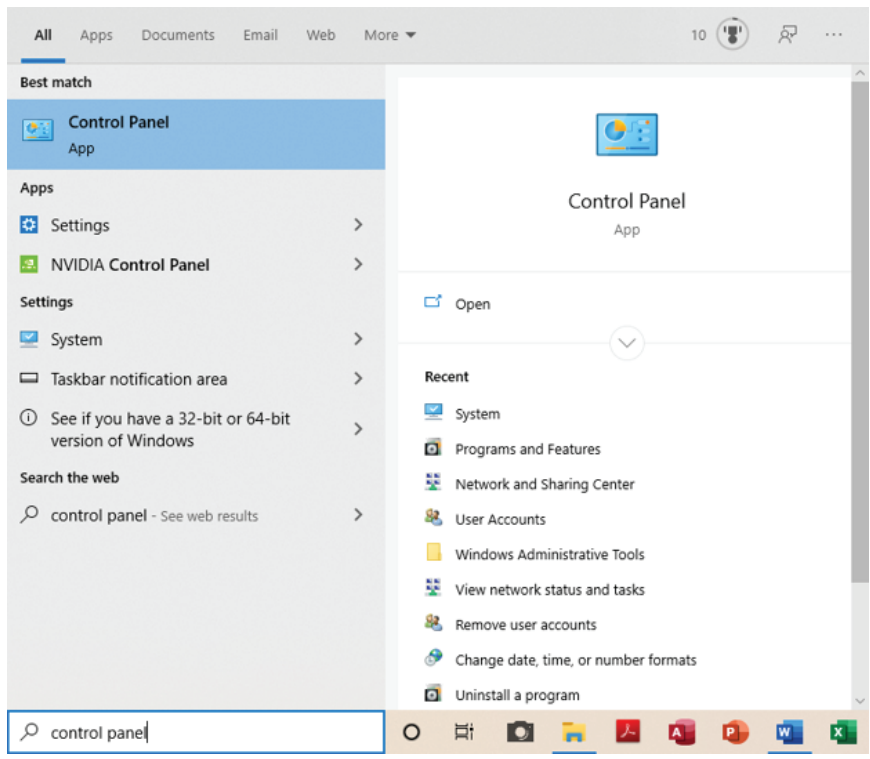

Figure 43. Accessing the Windows Control Panel using the Windows search tool.

2. Select "Clock and Region" (fig. 44), and then select "Change date, time, or number formats" (fig. 45).

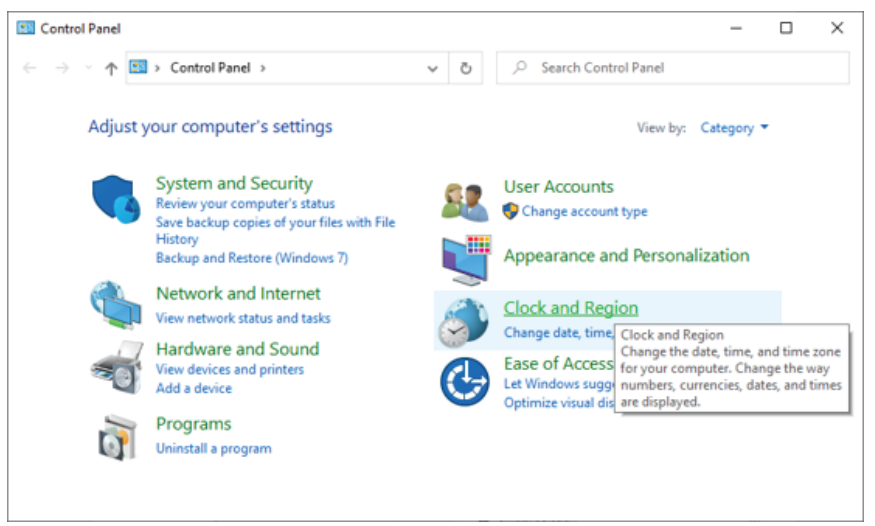

Figure 44. Selecting the Windows clock and region settings.

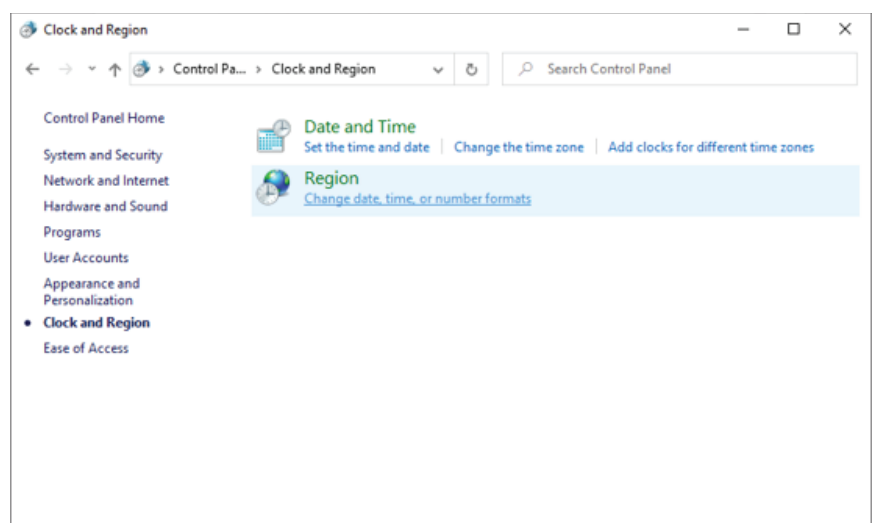

Figure 45. Selecting the "Change date, time, or number formats" option in Windows settings.

3. Select "Additional settings..." (fig. 46).

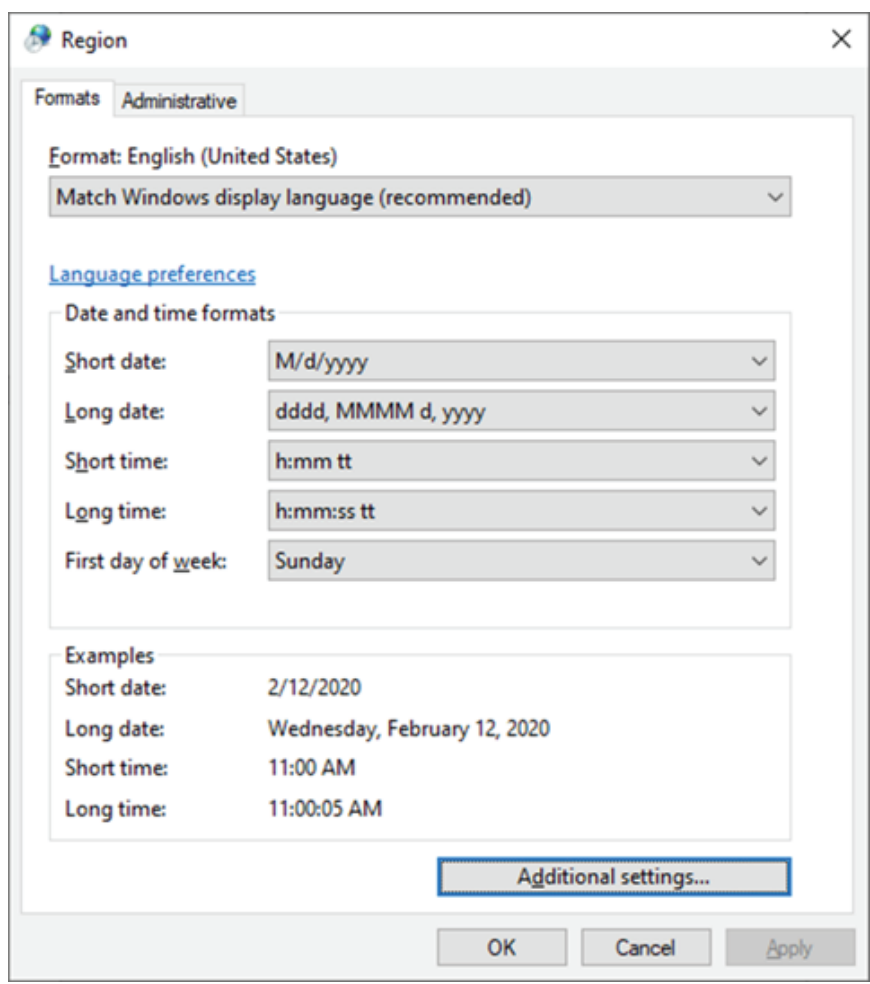

Figure 46. Selecting "Additional settings..." on the Windows region screen. 
4. Compare the values entered on the numbers tab (fig. 47) to those on the computer running SolVES and adjust them to match those shown in the figure and select "OK." Users should note the original settings on the numbers tab to ensure they are correctly restored. When finished using SolVES, the reset button on the numbers tab can be selected to restore the system default settings.

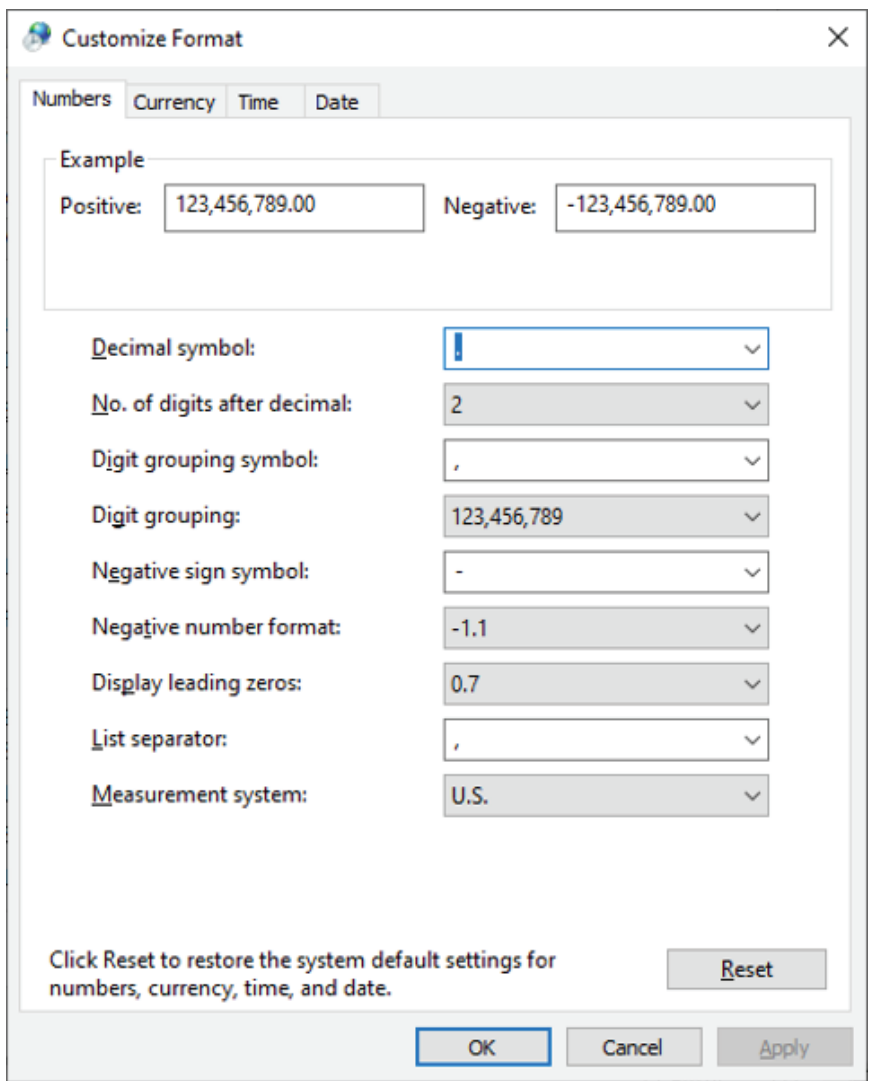

Figure 47. Numbers format for SolVES processing in Windows settings.

Users should now continue to the "Sample Data Installation" section.

\section{Sample Data Installation}

Installing the sample data allows the user to complete the demonstration analyses outlined in the "Project Setup," "Analyze Survey Data," and "Transfer Values" sections as well as to examine the results as outlined in the "View Results" section. These installation steps also apply to the unpopulated solves database included in the SolVES 4.0 download, which can be populated with the user's own survey and environmental data for analysis according to detailed instructions included in appendix 2.

1. Select the pgAdmin 4 application by entering "pgAdmin 4 " in the Windows search field and selecting the app (fig. 48).

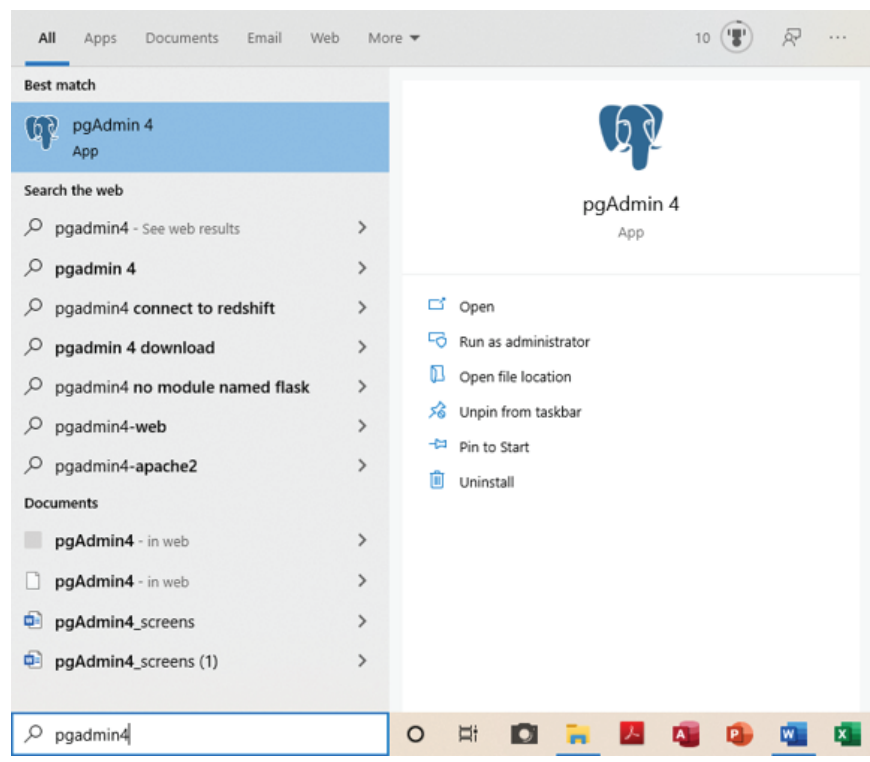

Figure 48. Accessing pgAdmin 4 using the Windows search tool.

2. After pgAdmin 4 opens in the browser window, select "Servers" in the browser tree to set a master password for pgAdmin 4 and select "OK" (fig. 49).

\section{Set Master Password}

Please set a master password for pgAdmin. This will be used to secure and later unlock saved passwords and other credentials Password $\quad$-........

$?$

$\mathbf{x}$ Cance

Figure 49. Entering a pgAdmin 4 master password. 
The pgAdmin 4 password is separate from the "postgres" password that will be used for connecting to PostgreSQL 11. Users may select a different master password, but they should keep a record of it. Users will not normally be prompted to enter this master password after this initial startup of pgAdmin 4.

3. Select the "PostgreSQL 11" server from the browser tree and enter the "postgres" password that was set during the PostgreSQL 11 installation process and select "OK" (fig. 50). Users may wish to check the box next to "Save Password," but it is not required.

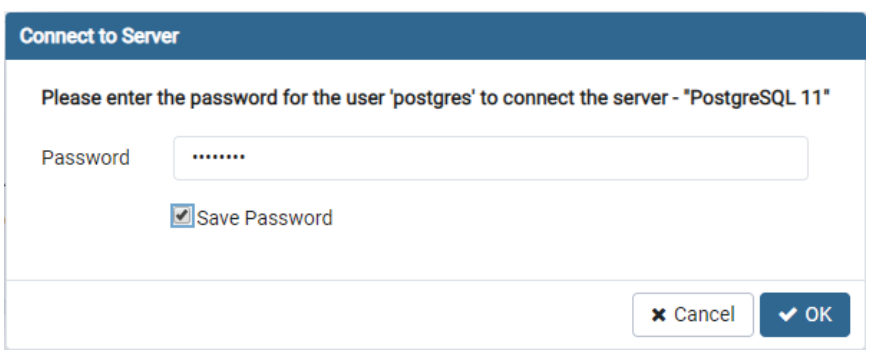

Figure 50. Entering the "postgres" password in pgAdmin 4.

4. Expand PostgreSQL 11 in the browser tree, right-click on "Databases," and select Create>Database... (fig. 51).

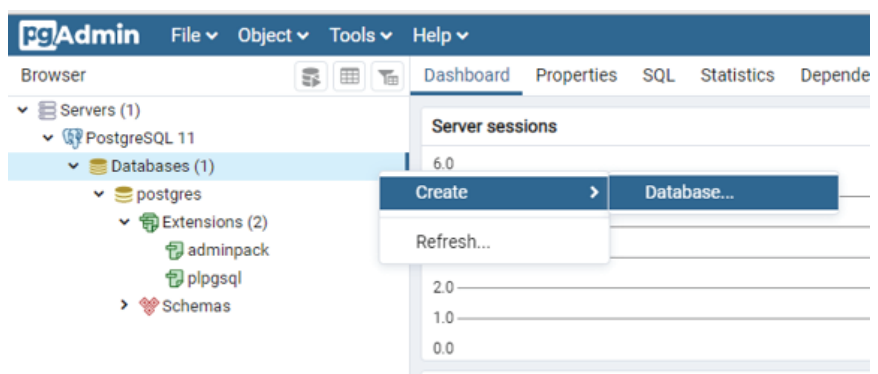

Figure 51. Creating database in PostgreSOL 11, pgAdmin 4.
5. Enter "solves" (must be all lowercase characters) in the database field on the general tab (fig. 52).

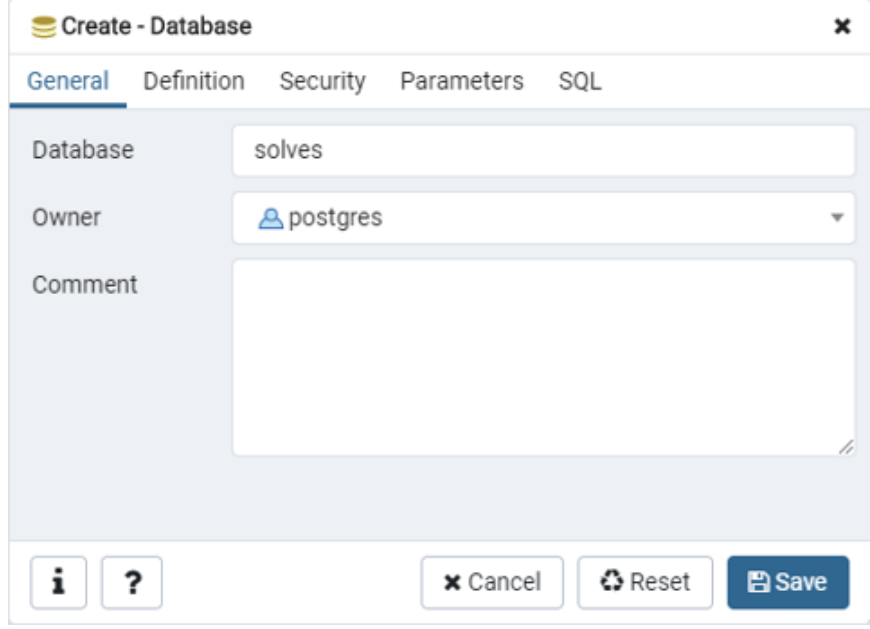

Figure 52. Creating the "solves" database in pgAdmin 4.

6. On the definition tab, set encoding to "UTF8" (if not already selected), tablespace to "pg_default," collation to "English_United States.1252," and character type to "English_United States.1252" and select "Save" (fig. 53).

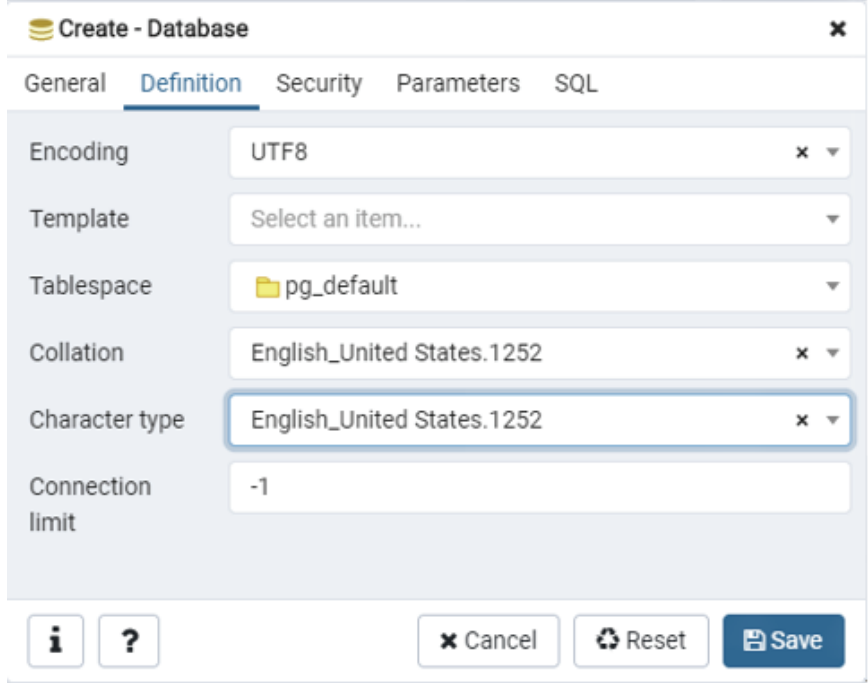

Figure 53. Setting the "solves" database definition in pgAdmin 4. 
7. Right-click on the newly created solves database and select "Restore..." (fig. 54) to open the "Restore" screen (fig. 55).

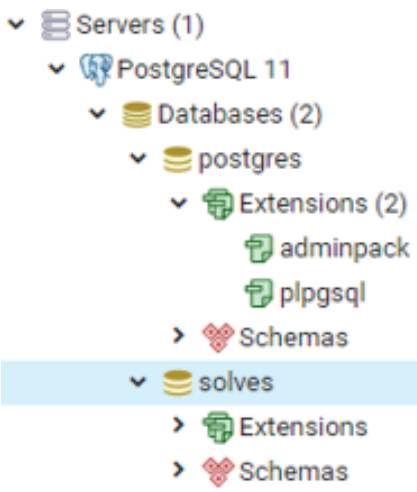

\begin{tabular}{|l|}
\hline Create \\
\hline Refresh... \\
Delete/Drop \\
CREATE Script \\
Disconnect Database... \\
\hline Maintenance... \\
\hline Backup.... \\
\hline Restore... \\
\hline Grant Wizard... \\
Query Tool... \\
Properties... \\
\hline
\end{tabular}

Figure 54. Restore data to "solves" database in pgAdmin 4.

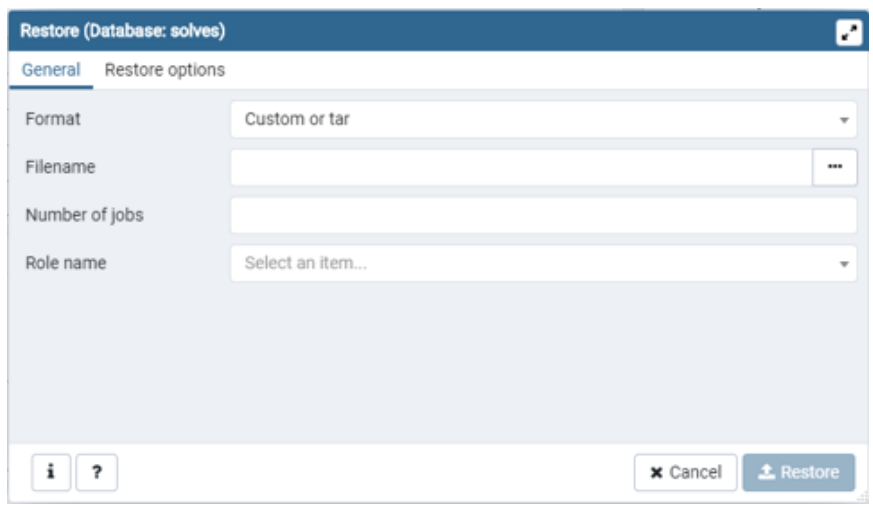

Figure 55. Database restore screen of pgAdmin 4.
8. Navigate to the Pike and San Isabel National Forests sample data "solves.backup" file included in the SolVES $\backslash$ DatalSample_Data $\backslash$ PSI directory and choose "Select" (fig. 56).

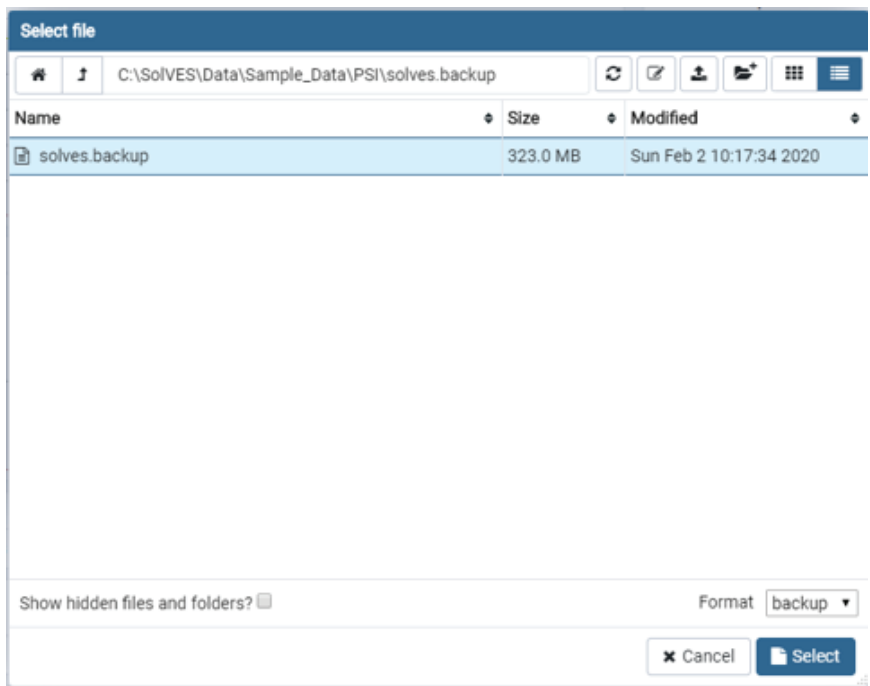

Figure 56. Selecting the "solves.backup" file in pgAdmin 4.

9. Select "Restore" (fig. 57).

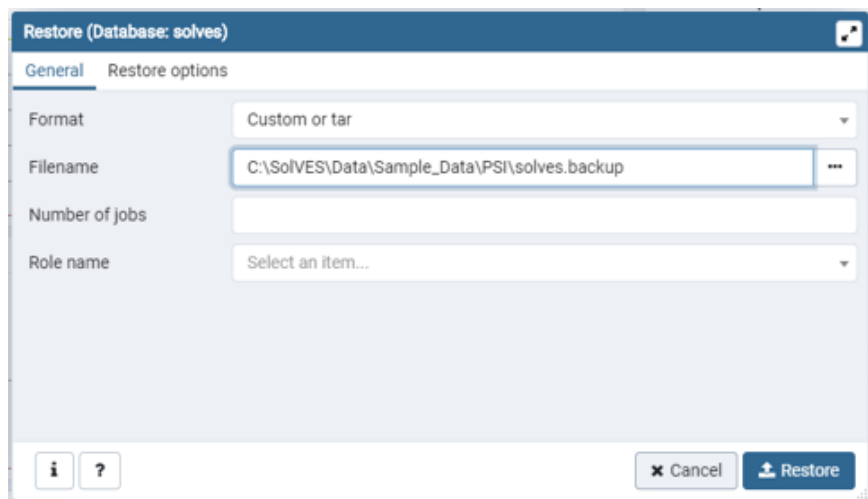

Figure 57. Database restore screen with selected "solves. backup" file in pgAdmin 4 . 
10. The database will be loaded with all Pike and San Isabel National Forests sample data (visible by expanding the "public" schema within the "solves" database) once the "Successfully Completed" message appears on screen (fig. 58).

\begin{tabular}{|c|c|c|}
\hline \multicolumn{3}{|c|}{ Restoring backup on the server } \\
\hline \multicolumn{3}{|c|}{ Restoring backup on the server 'PostgreSQL 11 (localhost:5432)' } \\
\hline \multicolumn{3}{|c|}{ Mon Aug 032020 13:19:08 GMT-0400 (Eastern Daylight Time) } \\
\hline (()) 85.48 seconds & (9) More details... & × Stop Process \\
\hline$\checkmark$ & fully completed. & \\
\hline
\end{tabular}

Figure 58. Successful completion of "solves" database restoration (loading of Pike and San Isabel National Forests sample data) in pgAdmin 4.

At this point, users can continue to the "Project Setup," "Analyze Survey Data," and "View Results" sections to complete the exercises using the Pike and San Isabel National Forests sample data. Alternatively, users can review appendixes 1 and 3 to learn more about SolVES 4.0.

If users wish to complete the steps in the "Transfer Values" section, they should return here to complete the following steps for updating the solves database with the Grand County sample data.

1. To complete the steps in the "Transfer Values" section using the Grand County sample data, right-click on the existing solves database and select "Delete/ Drop" (fig. 59). Confirm the deletion by selecting "OK" (fig. 60).

Note.-Alternatively, the existing solves database can be renamed by right-clicking on "Properties" and updating the database field on the general tab (for example "solves_1"). A new solves database can then be created for the Grand County sample data by continuing to step 2. SolVES 4.0 will access the data in the database currently named "solves." This renaming approach allows users to maintain multiple versions of solves databases without the need to repeat database installations.

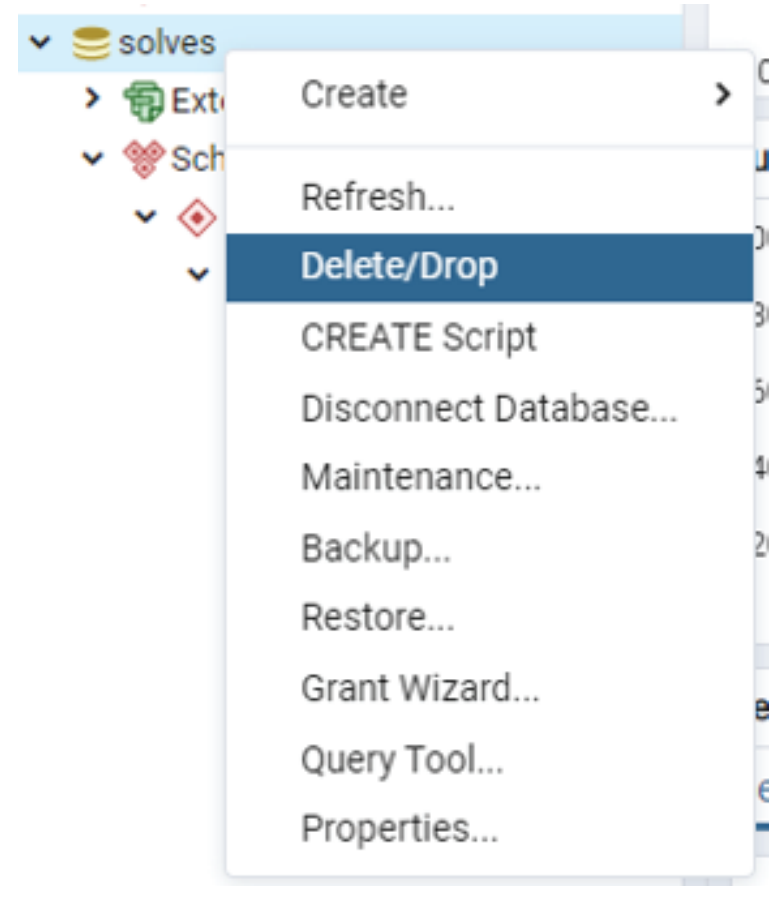

Figure 59. Delete/drop in the "solves" database in pgAdmin 4.

Drop Database?

Are you sure you want to drop database 'solves"?

x Cancel

Figure 60. Confirm deletion of existing "solves" database in pgAdmin 4.

2. Repeat steps $7-10$ as described for loading the Pike and San Isabel National Forests sample data but instead navigate to and restore the "solves.backup" file included in the $\backslash$ SolVES $\backslash$ Data $\backslash$ Sample_Data $\backslash$ Grand directory.

At this point, users can continue to the "Project Setup," "Transfer Values," and "View Results" sections to complete the exercises using the Grand County sample data. 


\section{Project Setup}

The following steps provide an example of creating a new project in SolVES 4.0.

1. To use SolVES 4.0, a new project must be started by selecting "Project Setup" from the SolVES plugin toolbar (fig. 61).

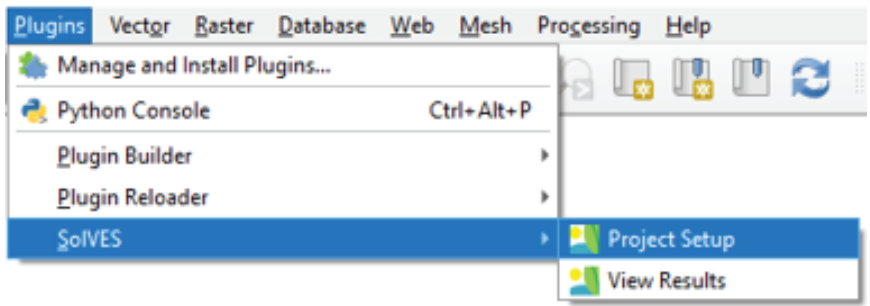

Figure 61. Creating a new project in Social Values for Ecosystem Services, version 4.0 .

2. Once the project setup form is open (fig. 62), navigate to the SolVES directory location to set the home directory (fig. 63). If the correct directory location is not entered, SolVES will return an error message and the correct directory location will need to be entered.

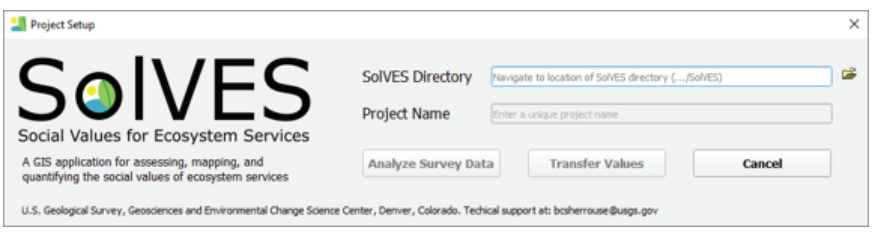

Figure 62. The Social Values for Ecosystem Services, version 4.0, project setup form.

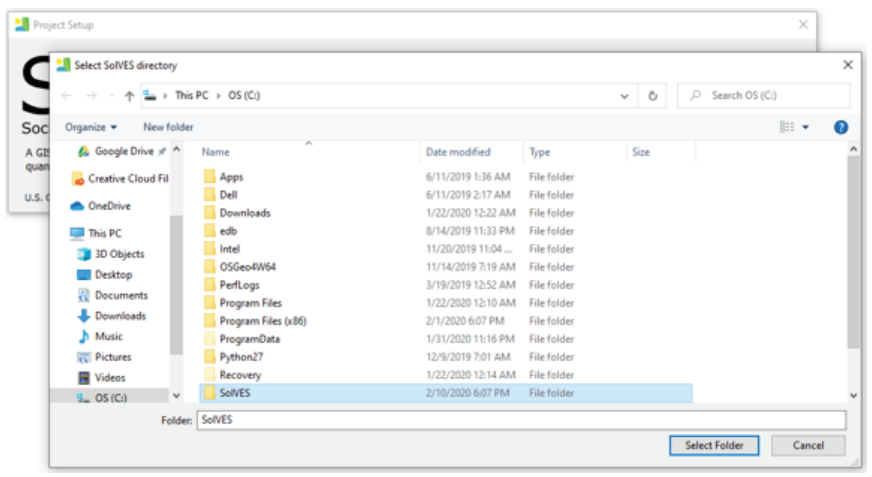

Figure 63. Navigating to the Social Values for Ecosystem Services, version 4.0, directory from the project setup form.
3. Enter a project name (fig. 64). This can simply be the name of the study area or some other unique, descriptive name. For the current exercise, enter "Pike and San Isabel National Forest." If the project name entered already exists, SolVES will return an error message and a new project name will need to be entered.

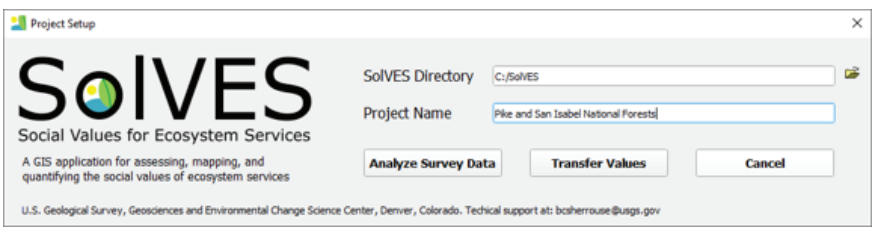

Figure 64. Populated Social Values for Ecosystem Services, version 4.0, project setup form.

Users can now proceed to the "Analyze Survey Data" section. 


\section{Analyze Survey Data}

The following steps provide an example of using the Analyze Survey Data tool to generate social-value maps for the Pike and San Isabel National Forests and provide descriptions of the options available for analyses.

1. Click the "Analyze Survey Data" button on the project setup screen to access the Analyze Survey Data tool (fig. 65), which provides access to the Ecosystem Services Social Values and Value Mapping Models.

2. Select the "By Survey Subgroup Across Social Value Types" radio button to analyze a specific survey subgroup.

Note.--If a user selects the "By Social Value Type Across Survey Subgroups" radio button, they will be able to choose a single social value type (for example, recreation) to compare between all available survey subgroups. Individual survey respondents can belong to more than one survey subgroup.

3. Set public use to "Motorized Recreation."

Note.- No value is required if all survey data are analyzed. A value must be selected if an "Attitude or Preference" value has been selected.

4. Set attitude or preference to "Oppose or Strongly Oppose."
Note.-A value must be selected if a public use value has been selected. No value is required if all survey data will be analyzed.

5. Accept the "Buffer Study Area" default option of "No."

Note.-If a buffer is desired, the option can be set to "Yes" and a buffer width (in meters) must then be entered. Buffers allow users to include data in their analysis that fall just outside the formal study area boundary.

6. Accept the "Weight Survey Points" default option of "Yes."

Note.--If no allocation data are available or a user chooses to generate unweighted results, the option can be set to "No."

7. Accept the "Threshold Features" default option of "Yes."

Note.- Including threshold features in an analysis generally results in models with higher AUC values. However, if the social-value maps generated by an analysis display discontinuities in the distribution of the value index (that is, abrupt changes in social-value intensity that are not determined to be realistic within the context of a study area), the option can be set to "No."

8. Enter " 450 " as the output cell size (in meters).

Analyze Survey Data - Select Model Options

Model Options

Return to Project Setup to create new projects for other survey subgroups, social value types, or model parameters.

Value Comparison Type

- By Survey Subgroup Across Social Value Types Leave Public Use and Attitude or Preference blank to analyze results from all surveys. Must select both to analyze subgroups.

Public Use

Attitude or Preference

By Social Value Type Across Survey Subgroups

Individual respondents can belong to more than one survey subgroup.

Social Value Type

Buffer Study Area $\bigcirc$ Yes $\bullet$ No
Weight Survey Points $\bullet$ Yes $\bigcirc$ No
Threshold Features $\bullet$ Yes $\bigcirc$ No

Threshold Features $\bullet$ Yes No

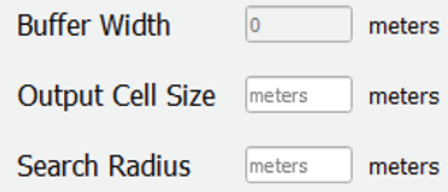

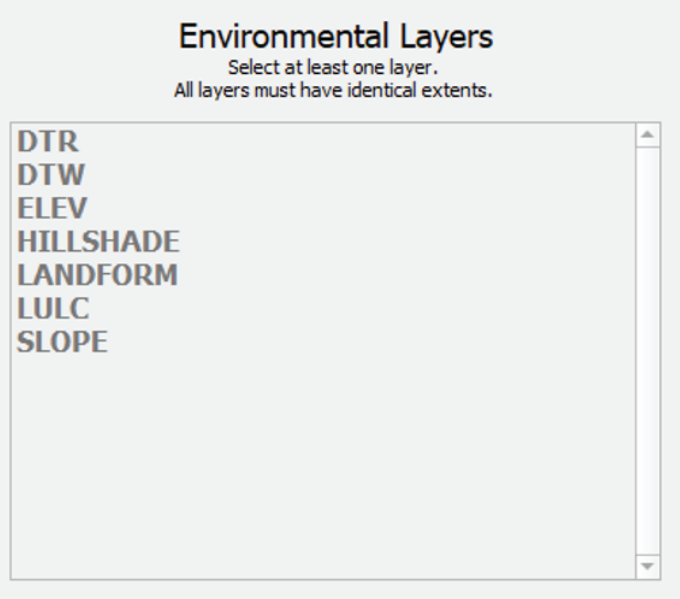

Environmental Layers

Select at least one layer.

DTR

DTW

HILLSHADE

LANDFORM

LULC

SLOPE

$\checkmark$
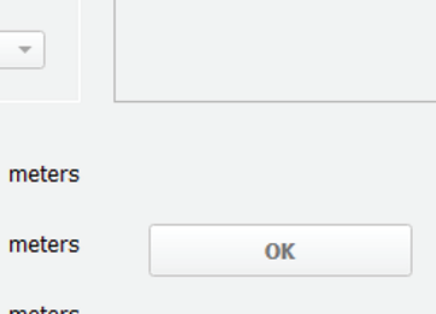

Figure 65. The Analyze Survey Data tool of Social Values for Ecosystem Services, version 4.0. 
Note.-This parameter is usually determined by the scale of the map or maps used in a survey. A general rule of thumb is to apply a cell size that is one thousandth the value of the denominator of the survey map scale. For example, if the map scale is 1 to 450,000 , then the output cell size is set to 450 meters $(\mathrm{m})$. The assumption is that a hand-marked point on the map is approximately 1 millimeter $(\mathrm{mm})$ in width.

9. Accept the default search radius of " 4500 " (in meters) that appears after entering the output cell size.

Note.-The search radius is used by the kernel density function and defaults to 10 times the output cell size. Users can enter a different search radius value if the default value is not adequate for a specific analysis. Larger values will generate more generalized kernel density surfaces.

10. Select DTR, DTW, ELEV, LANDFORM, LULC, and SLOPE from the environmental layers list (fig. 66).

Note.-The list will not become enabled until after the output cell size has been populated. Multiple layers can be selected by holding down the CTRL key or the SHIFT key while making selections. If any options or parameters are changed after selecting the environmental layers, the environmental layers will need to be selected again.

11. After all options and parameters have been set (fig. 66), select the now-enabled "OK" button.

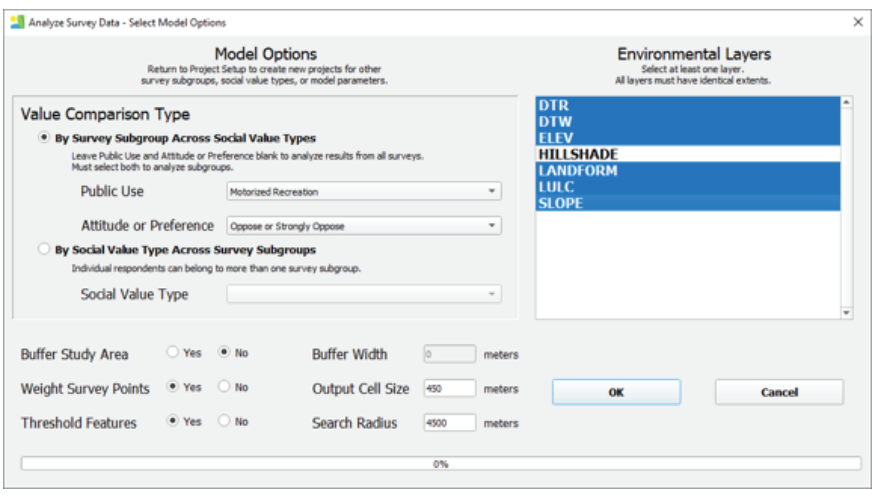

Figure 66. The Analyze Survey Data tool ready to process the Ecosystem Services Social-Values Model of Social Values for Ecosystem Services, version 4.0.

The Ecosystem Services Social-Values Model will process as described below (fig. 67). Processing may take several minutes, and a message may temporarily appear in the screen's title bar indicating that SolVES is not responding.

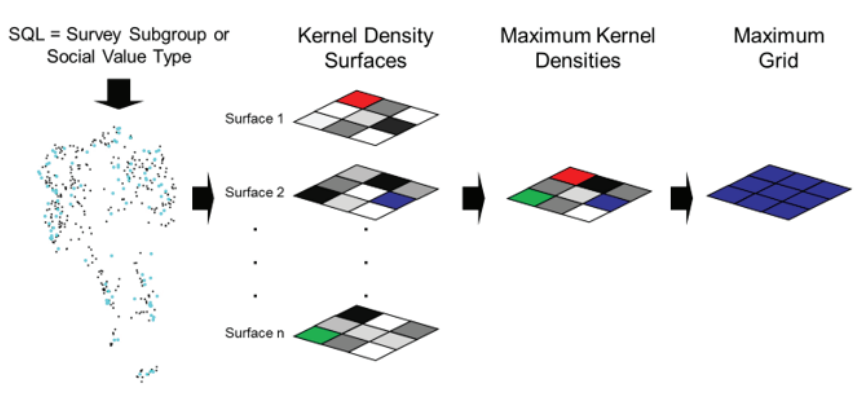

Figure 67. Ecosystem Services Social-Values Model generalized process flow of Social Values for Ecosystem Services, version 4.0.

The survey subgroup (public use and attitude or preference) parameters (or the social-value type parameter depending on the selected value comparison type) are converted to a Structured Query Language (SQL) statement, which is then used to select the corresponding mapped survey points. Kernel-density surfaces weighted by the total amount of value allocated to each social-value type (or not weighted if the "Weight Survey Points" option is set to "No") are generated for each social-value type (or each survey subgroup). The values contained in every cell are compared among all kernel density surfaces, and the maximum kernel density value at each cell location is identified. The red, blue, and green cells indicated in figure 67 represent examples of maximum kernel density values identified at those cell locations within those surfaces (for example, the red cell indicates that the highest value at that cell location was found in surface 1). The overall maximum kernel-density value among all these surfaces is identified (for example, in figure 67, this overall maximum value was in the blue cell in surface 2). This "Maximum Grid" will be used by the Value Mapping Model to normalize the kernel-density surfaces and to ultimately generate social-value maps. In addition, during model processing, the selected mapped points are converted to a comma-separated values (CSV) file, and the environmental layers are resampled according to the user-provided output cell size and converted to American Standard Code for Information Interchange (ASCII) format for use by Maxent.

The kernel-density surfaces are generated following a method like that of Alessa and others (2008) in their mapping of social-ecological hotspots on Alaska's Kenai Peninsula. As opposed to simple point density, the basis of kernel density is a quadratic kernel function (Silverman, 1986). This function defines a smoothly curved surface fit over each point and extending out to a defined search radius. The volume below each surface is equal to the weight assigned to the point.

To assist users with the selection of social-value types of survey subgroups for further analysis by the Value Mapping Model, the Ecosystem Services Social-Values Model generates average nearest-neighbor statistics describing the relative dispersion, clustering, or randomness of the mapped points. Following the example of Clement (2006) and Brown and others (2002), the point data are subjected to Completely 
Spatially Random hypothesis testing through the calculation of average nearest neighbor statistics. The ratio of the observed distance between points to the expected distance between points is represented by an $R$ value. Each $R$ value, along with its number of standard deviations from the mean, or $Z$ score, identifies point patterns for which statistically significant clustering is observed. Such clustering is indicated by $R$ values of less than 1 and large negative $Z$ scores. Average nearest neighbor statistics are calculated based on the total area within the study area boundary. These statistics can be used to limit a user's focus to social-value types occupying locations with specific levels of significance. Individual users may be less concerned about the relative spatial distribution of the mapped points, but the average nearest neighbor statistics do provide an initial indication of the social-value maps that will likely display the most intensely valued hotspots.

Once processing of the Ecosystem Services SocialValues Model is complete, the second Analyze Survey Data tool screen is opened to provide access to the Value Mapping Model. A list of all social-value types (when the "By Survey Subgroup Across Social Value Types" value comparison type is selected) or all survey subgroups (when the "By Social Value Type Across Survey Subgroups" value comparison type is selected) available for analysis is presented, along with their associated point counts and average nearest neighbor statistics. An example showing user-selected, social-value types is shown below (fig. 68).

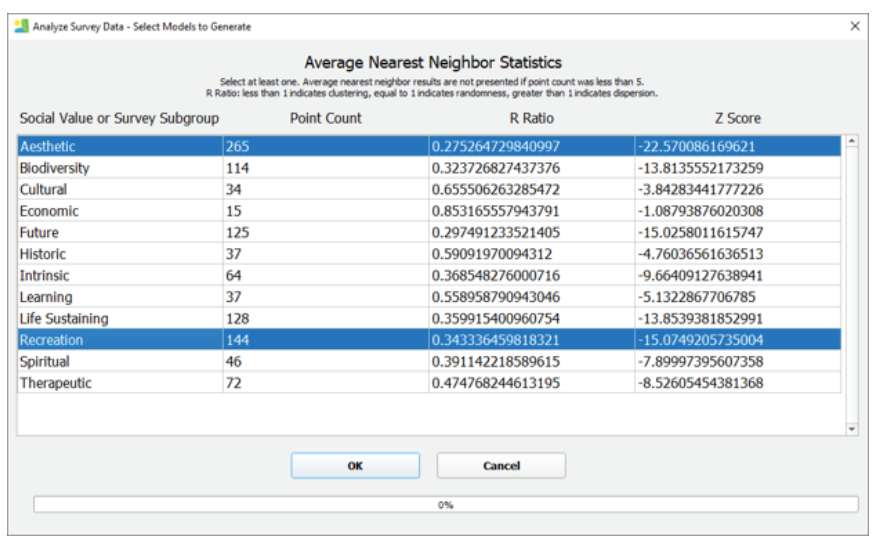

Figure 68. The Analyze Survey Data tool ready for processing the Value Mapping Model of Social Values for Ecosystem Services, version 4.0.

1. Select the "Aesthetic" and "Recreation" social values.

Note.-Users can make selections on the basis of average nearest neighbor statistics or other criteria. At least one social-value type or survey subgroup must be selected. Multiple items can be selected by holding down the CTRL key or the SHIFT key while making selections. Because of how SolVES instructs Maxent to partition points into training and test data, results will not be presented for any entry having less than 5 points.

\section{Select the now-enabled "OK" button.}

Processing may take several minutes, and a message may temporarily appear in the screen's title bar indicating that SolVES is not responding. The model will process as described below in figure 69.

Given the selected social-value types or survey subgroups, the Value Mapping Model identifies the matching kernel-density surfaces that were generated by the Ecosystem Services Social-Values Model. The selected kernel-density surfaces are then divided by the maximum grid generated by the Ecosystem Services Social-Values Model. The normalized grids are standardized into the 10-point, kernel-density based value-index integer grid, and the value index maximum for each grid is identified. The Value Mapping Model then sends processing instructions to Maxent by means of a batch file. Maxent uses the specially formatted point and environmental data converted by the Ecosystem Services Social-Values Model to generate logistic value layers (ranging from 0 to 1) for each selected social-value type or survey subgroup. Maxent also generates models as lambdas files for each selected social-value type or survey subgroup based upon the relationship between the mapped points and the environmental layers. Included along with these models are AUC statistics, indicating the goodness of fit of each model for the study area and their potential performance in transferring social values to similar areas. The Value Mapping Model converts Maxent's logistic output to social-value maps of the final value-index surface (ranging from 0 to 10) by multiplying each value layer with its corresponding value index maximum and rounding the result to the nearest integer. Finally, the Value Mapping Model uses the social-value maps generated from the Maxent output to calculate zonal statistics (that is, the environmental metrics) from each environmental layer.

Given the range of values included in the Maxent logistic output, the process of multiplying the value index maximum by the logistic output possibly will result in the final maximum attained index value being reduced to the next lowest integer value. For example, if the value index maximum for a particular social-value type is 10 and the maximum value in the logistic output is less than 0.95 , the resulting value-index surface will have a maximum value of 9 . Also, the minimum value in the logistic output can be possibly greater than or equal to 0.05 , resulting in final value-index surfaces having a minimum value greater than 0 . 


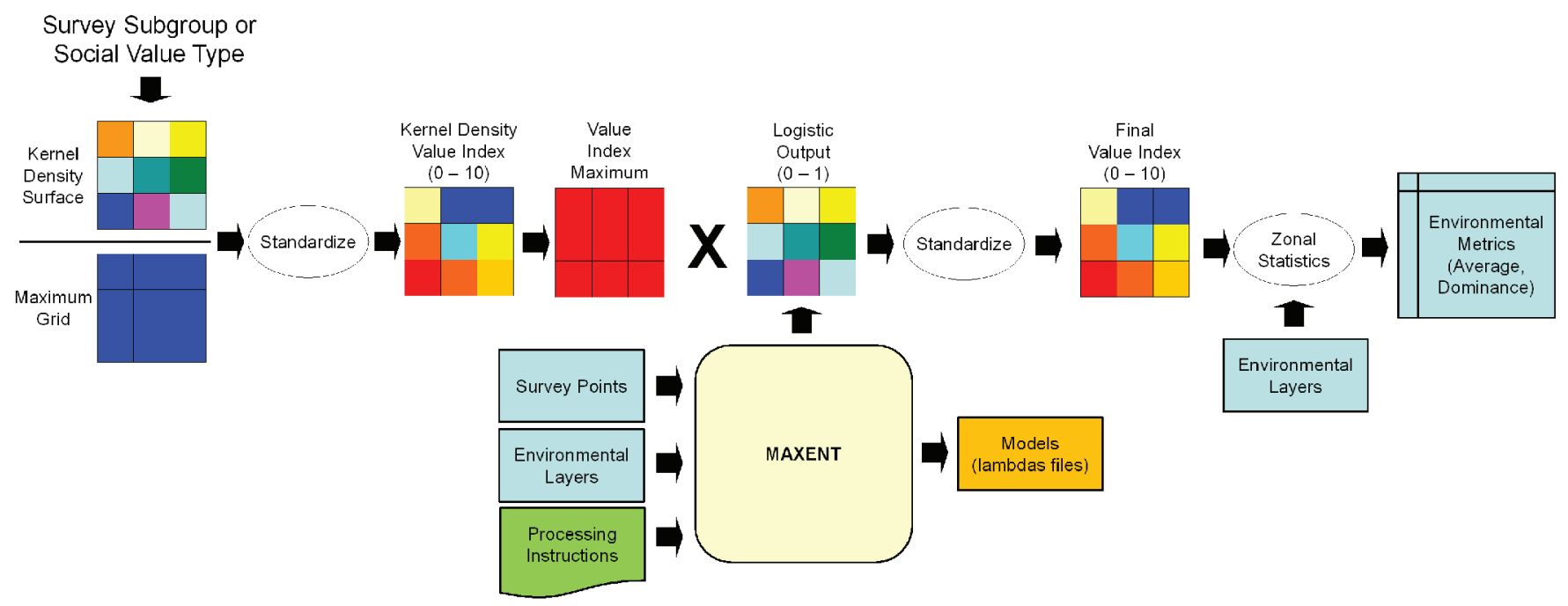

Figure 69. Value Mapping Model generalized process flow of Social Values for Ecosystem Services, version 4.0.

\section{Calculation and Interpretation of the Value Index}

As a result of normalizing to the maximum grid, the value of every cell on every kernel density grid is scaled relative to the highest kernel density value among all social-value types or survey subgroups (values ranging from 0 to 1 ). This normalization, however, can result in highly positively skewed value distributions. This can limit the usefulness of socialvalue maps generated for all but a few of the most highly rated social-value types. To counter this effect and thereby improve the utility of a greater number of social-value maps produced during an analysis, the square root of each normalized grid is taken as part of the standardization process that converts the normalized grid to the kernel-density based, value-index surface. Because the normalized grids have dimensionless values ranging from 0 to 1 , the resulting transformation is monotonic.

The value index can be used to measure and compare the magnitude of value differences across social-value types using the "By Survey Subgroup Across Social Value Types" value comparison type and survey subgroups using the "By Social Value Type Across Survey Subgroups" value comparison type. For analysis of multiple social-value types across a single survey subgroup, the higher the value attained on the value index by a social-value type, the more highly it is valued by that survey subgroup. A social-value type that attains a 10 on the value index corresponds to one or more locations within the study area where that survey subgroup values that socialvalue type more highly than at any other location and more highly than any of the other social-value types, regardless of location. For social-value types that attain less than a 10 on the value index, the value corresponds to locations where that social-value type is valued more highly than at any other location by that survey subgroup. For analysis of a single socialvalue type across multiple survey subgroups, a value index of 10 will indicate the survey subgroup that most highly values the selected social-value type. Because survey respondents can belong to more than one survey subgroup, relative value, as measured by the value index, will be sensitive to the number of respondents who are members of each subgroup. The maximum value attained on the value index will tend to increase as the relative size of the subgroup increases. This sensitivity to subgroup size should be kept in mind when making comparisons across subgroups for a single social-value type. An example of the Analyze Survey Data tool when the "By Social Value Type Across Survey Subgroups" value comparison type has been selected is shown below (fig. 70).

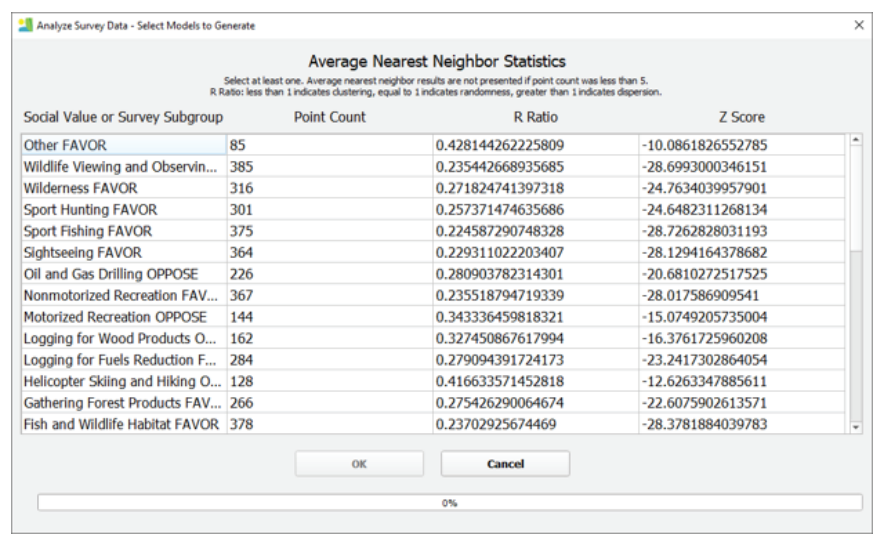

Figure 70. Example of survey subgroups when the Analyze Survey Data tool "By Social Value Type Across Survey Subgroups" value comparison type is used in Social Values for Ecosystem Services, version 4.0.

A new project must be created each time the Analyze Survey Data tool is used. This would include generating models for a different survey subgroup or other model parameters (for example, weighting, buffers, included environmental layers). This prevents previous results from being overwritten. 


\section{Transfer Values}

The Value Transfer Mapping Model, accessible through the Transfer Values tool, can be used when survey data are unavailable for a study area having similar biophysical and social contexts to a primary study area. It relies on statistical models generated by Maxent from previous SolVES analyses in study areas having available survey data. For more information regarding the SolVES value-transfer implementation, see Sherrouse and Semmens (2014). Before running the Transfer Values tool, the individual social-value model's metadata should be reviewed to acquire the appropriate environmental data, to identify the necessary parameter values for the Transfer Values tool, and to determine if the model's physical and social context is sufficiently similar to the receiving study area for value transfer to be a viable option (Semmens and others, 2019). For the purposes of completing the exercise in this section, reviewing the metadata is not required. An example of the metadata can be viewed by using Windows to navigate to the $\backslash$ SolVES $\backslash$ Models $\backslash$ Pike and San Isabel National Forests $\backslash A 1 l$ Surveys $\backslash$ metadata directory (fig. 71) and viewing the contents of the Hypertext Markup Language (HTML) files (fig. 72).

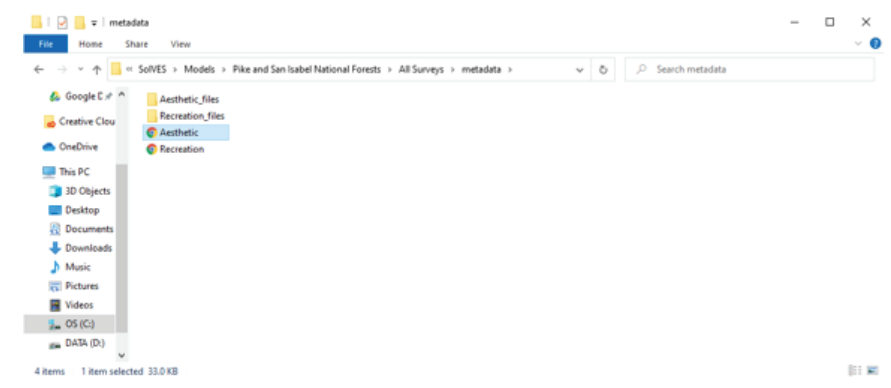

Figure 71. Navigating to the location of the models and metadata for use with the Transfer Values tool.

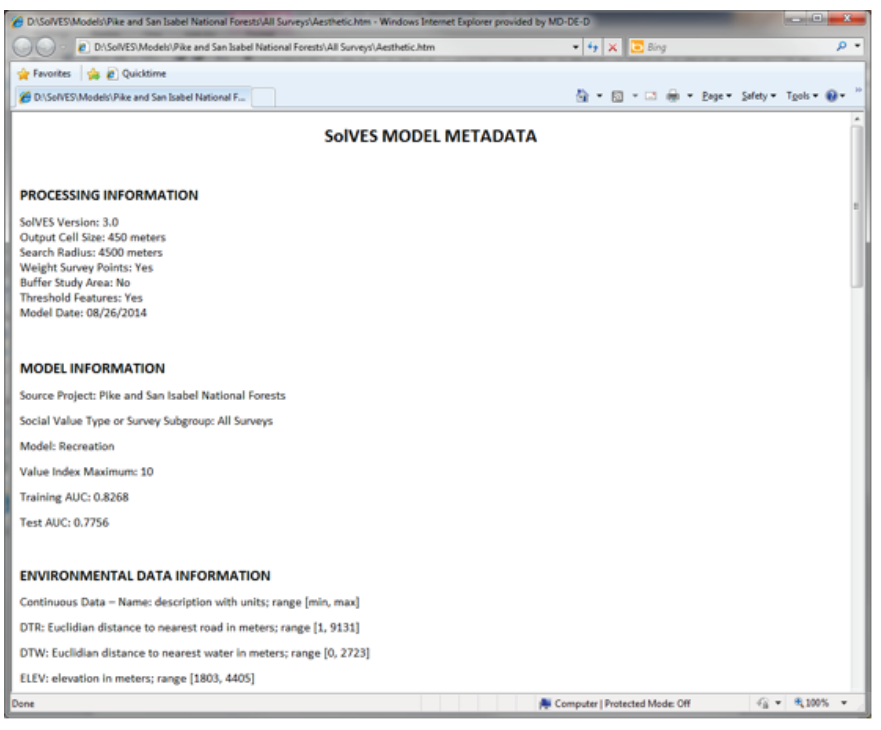

Figure 72. Partial contents of a value transfer model metadata file.
Although lambdas files contain the actual models, the matching HTML files include the original model processing parameters and information, descriptions of the environmental data used to generate the models, graphs illustrating the modeled relation among the environmental data and the value index, and any available information regarding the model's social context such as socioeconomic and demographic summaries. Please note the social-value models and associated metadata included with the SolVES 4.0 download are meant for demonstration only.

The following steps provide an example of using the Transfer Values tool to apply a model from the Pike and San Isabel National Forests to Grand County, Colorado. If a solves database containing the Grand County sample data has not already been created, review the "Sample Data Installation" section before proceeding.

1. Open the SolVES project setup screen.

2. Set the SolVES directory location.

3. Enter the project name "Pike San Isabel to Grand County Value Transfer" (fig. 73).

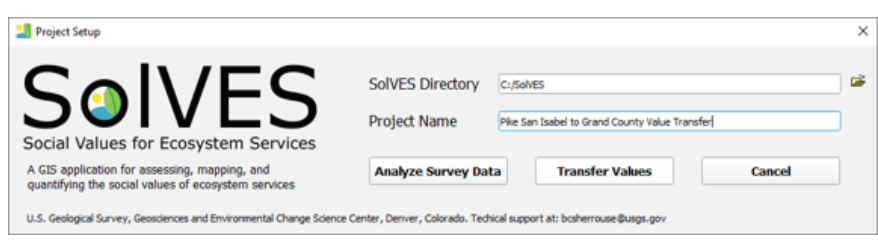

Figure 73. Setting up a transfer values project in Social Values for Ecosystem Services, version 4.0.

4. Open the Value Transfer Mapping Model (fig. 74) by clicking the "Transfer Values" button on the project setup screen.

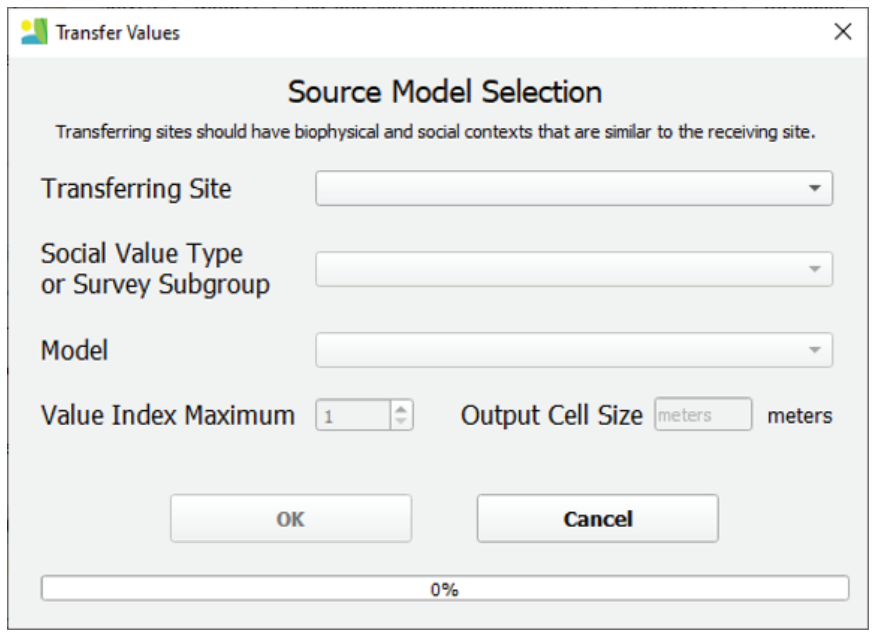

Figure 74. The Transfer Values tool of Social Values for Ecosystem Services, version 4.0. 
5. Select "Pike and San Isabel National Forests" as the transferring site.

6. Select "All Surveys" as the social value type or survey subgroup.

7. Select "Aesthetic" as the model.

8. Select " $10 "$ as the value index maximum.

9. Enter " 450 " as the output cell size.

10. The Transfer Values tool screen should look like the example shown in figure 75. Select "OK."

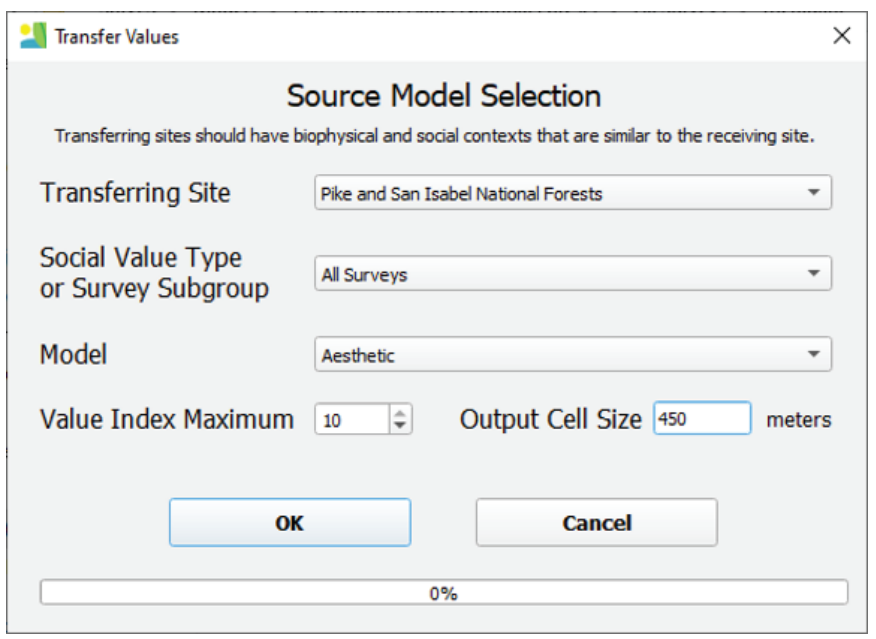

Figure 75. The Transfer Values tool screen after parameter selection in Social Values for Ecosystem Services, version 4.0.

The model will process as shown in figure 76. Processing may take several minutes.
The transferring site and social value type or survey subgroup selections navigate the user through the project-level and analysis-level folders contained in the models folder to the selected model. The selected model informs the Transfer Values tool which lambdas file to select. The selected environmental layers are resampled to the designated output cell size, and the Value Transfer Mapping Model sends processing instructions to Maxent as a batch file. Maxent applies the selected lambdas file to the resampled environmental layers to generate a logistic value layer for the selected model. The Value Transfer Mapping Model then multiplies the resulting logistic value layer by the selected value-index maximum and standardizes the result to a social-values map of the final value-index surface (ranging from 0 to 10 ). Lastly, the socialvalues map is used to calculate metrics from each environmental layer.

If a new model is generated that a user wishes to apply with the Transfer Values tool, the lambdas file for that model should be placed inside the $\backslash$ SolVES $\backslash$ Models folder within subfolders bearing the names of the transferring site and the social value type or survey subgroup (for example, |SolVES $\backslash$ Models\Pike San Isabel National Forest\All Surveys\Aesthetic. lambdas).

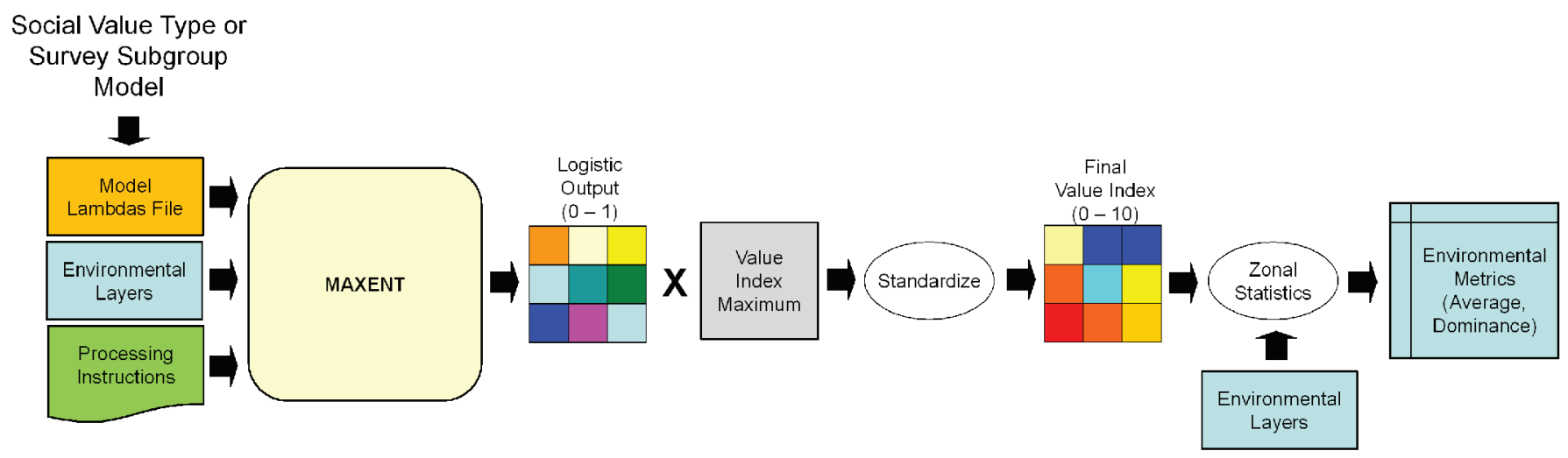

Figure 76. Value Transfer Mapping Model generalized process flow of Social Values for Ecosystem Services, version 4.0. 


\section{View Results}

The View Results tool allows users to produce composite reports of social-value maps and graphs of associated environmental metrics from the results of a current project or from previously completed projects. Complete the following steps to view results generated by SolVES.

1. Select "View Results" from the SolVES toolbar (fig. 77) to access the tool (fig. 78).

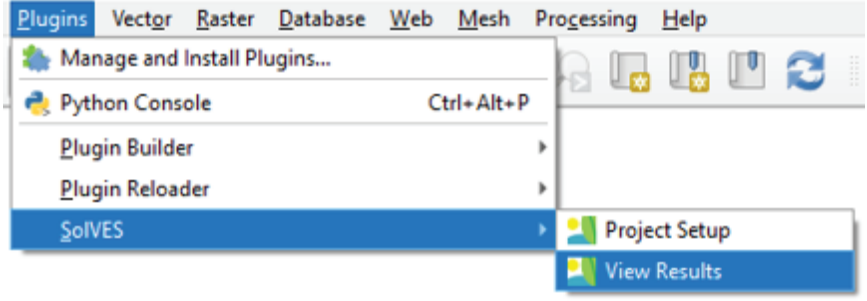

Figure 77. Selecting the View Results tool in Social Values for Ecosystem Services, version 4.0.

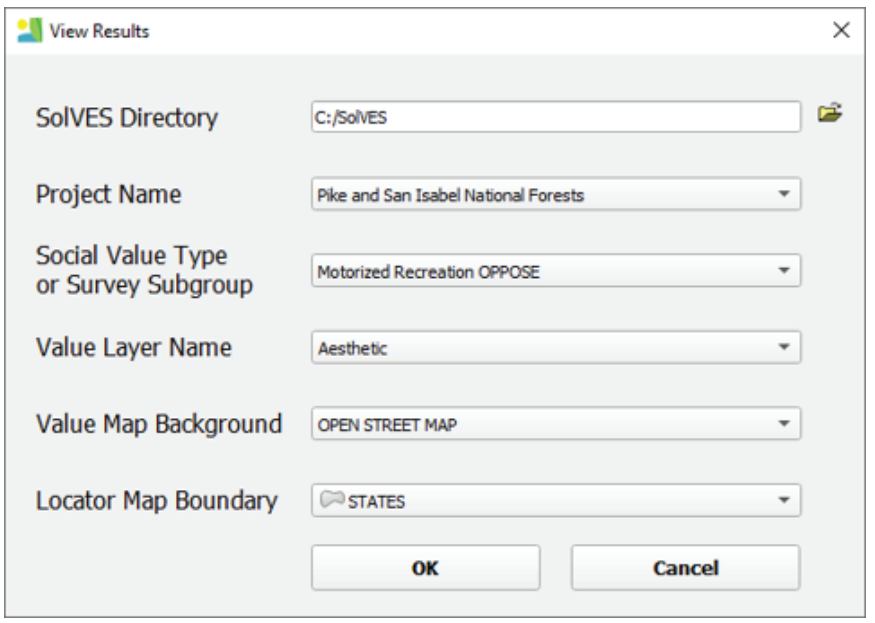

Figure 78. Accessing and populating the View Results tool in Social Values for Ecosystem Services, version 4.0.

2. Navigate to the SolVES directory.

3. Select "Pike and San Isabel National Forests" as the project name.

4. Select "Motorized Recreation OPPOSE" as the social value type or survey subgroup.

5. Select "Aesthetic" as the value layer name.

Note.-All value layers generated for a single project will be available for selection.
6. Select "OPEN STREET MAP" as the value map background.

Note.-A background is not required. All raster layers included in the solves database are available to use as a background. SolVES 4.0 also includes a new option to select OpenStreetMap as the background.

7. Select "STATES" as the locator map boundary.

Note.-A boundary is not required. All polygon layers included in the solves database are available to use as a boundary.

8. Select "OK." Within a few seconds, a composite report will be generated in the map layout (fig. 79).

The results generated from the exercise in the "Transfer Values" section can also be viewed using the View Results tool by repeating the previous steps but substituting project name with "Pike San Isabel to Grand County Value Transfer" and social value type or survey subgroup with "All Surveys" (fig. 80).

The map layout contains the selected value index map, including the study area boundary and selected background. The map title includes the project name, survey subgroup, and social-value type. The order of the survey subgroup and social-value type are reversed when the "By Social Value Type Across Survey Subgroups" value comparison type is selected. The locator map in the upper right corner indicates the location of the study area within a larger geographic region. Graphs of environmental metrics (up to 12) are included on the second page of the map layout. Continuous data are displayed as line graphs. Categorical data are displayed as scatterplots. The $\mathrm{x}$-axis labels for the scatterplots have the integer value designating the category. The categorical layer's original source dataset or metadata should be consulted if the meaning of the integer value is not known.

The AUC values, average nearest neighbor statistics, and the maximum value index score are included in the map layout for all results generated by the Analyze Survey Data tool. Only the maximum value index score is displayed for results generated by the Transfer Values tool. The transferred model's metadata should be referenced for additional information.

The map layout produced by the View Results tool can be manipulated to suit an individual user's needs. Titles can be modified, and map and graph elements can be moved and resized within the layout document. The desired map layout items will need to be unlocked first using the Items panel (see the panel at right side of layouts in figs. 79 and 80). Additional data layers can be turned on or added using the QGIS layers panel. A time stamp is included on the map layout to document when it was created. Map layouts can be exported as images or Portable Document Format (PDF) files using the export commands located in the layout menu. 

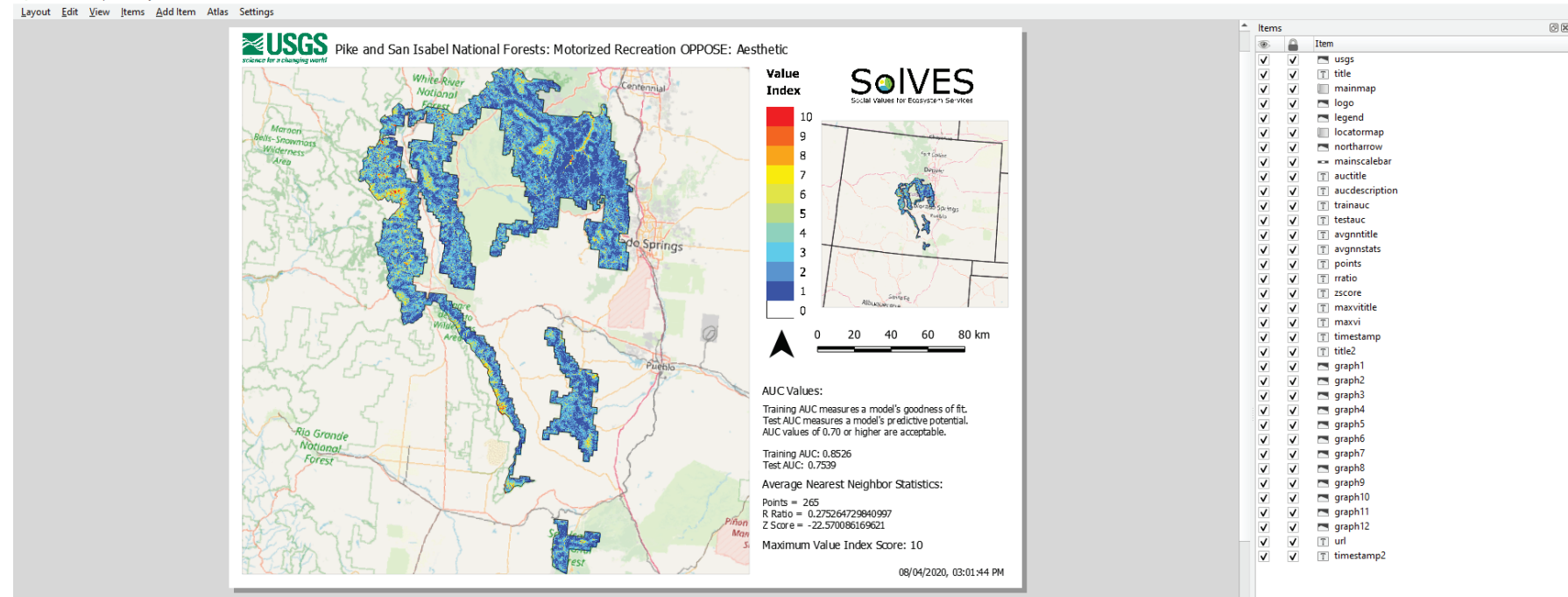

ॠख

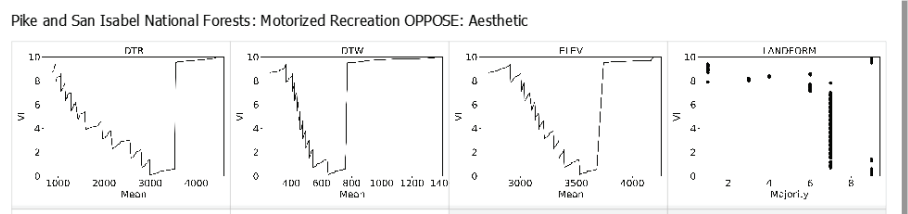

Figure 79. View results from the Analyze Survey Data tool in Social Values for Ecosystem Services, version 4.0.

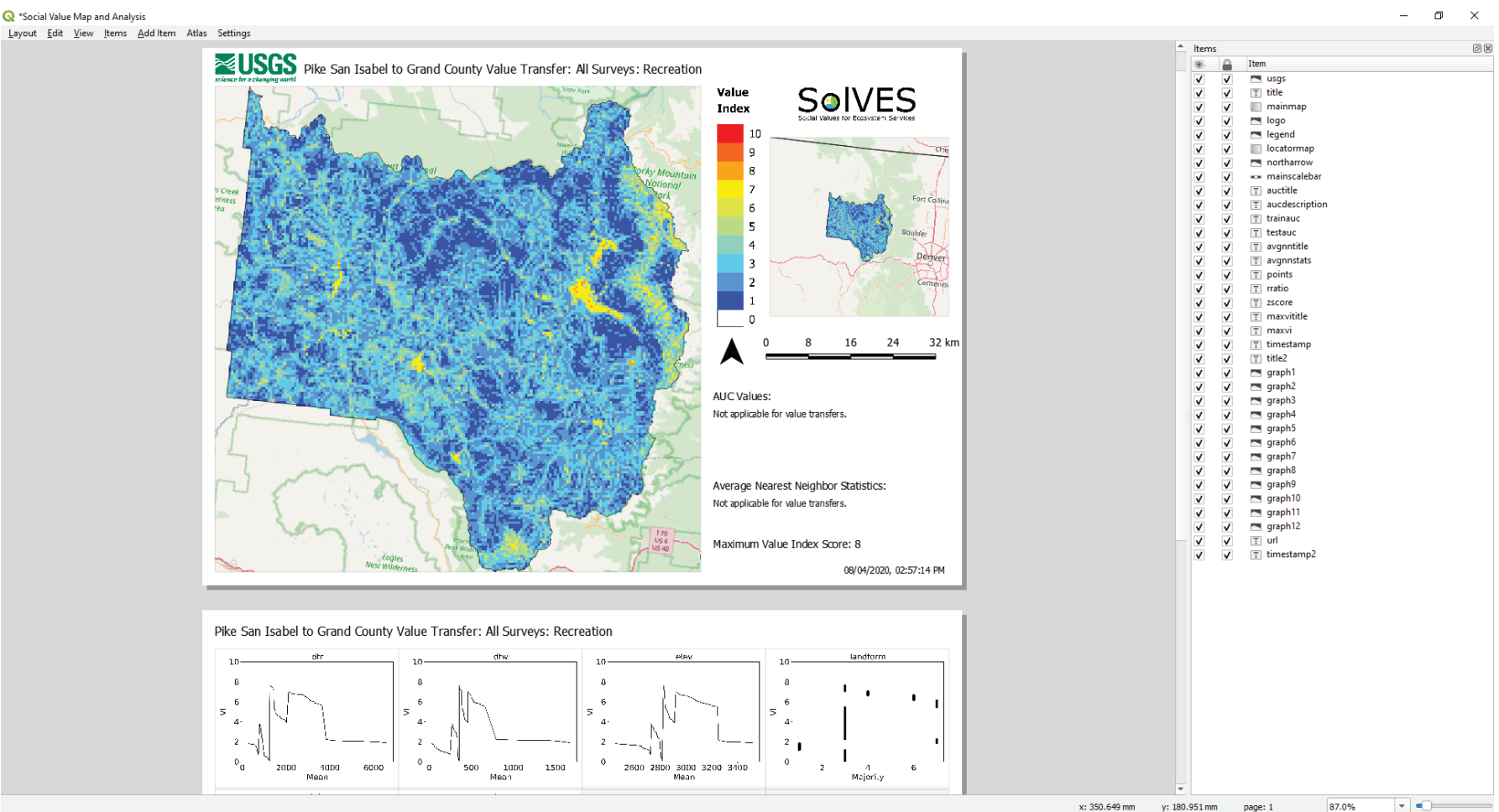

Figure 80. View results from the Transfer Values tool in Social Values for Ecosystem Services, version 4.0. 


\section{Interpreting and Adjusting Maxent's Area Under the Curve Values and Variable Contributions}

When the SolVES Analyze Survey Data tool is used, Maxent produces a model for each user-selected social-value type or survey subgroup. Along with these models, Maxent produces additional statistics describing the performance of each model. Included in these is the AUC statistic. The AUC statistic represents the area under the Receiver Operating Characteristic (ROC) curve. This is a curve in which the false positive rate (specificity or commission error) of predicted class membership is plotted on the $\mathrm{x}$-axis and the true positive rate (sensitivity or omission error) is plotted on the y-axis. Maxent does not rely on true absence points, so the ROC curves it generates plot fractional predicted area on the x-axis, which considers the fact that Maxent is analyzing presencerandom data instead of presence-absence data. An example of a ROC curve generated by Maxent and included in its HTML output is shown in figure 81 .

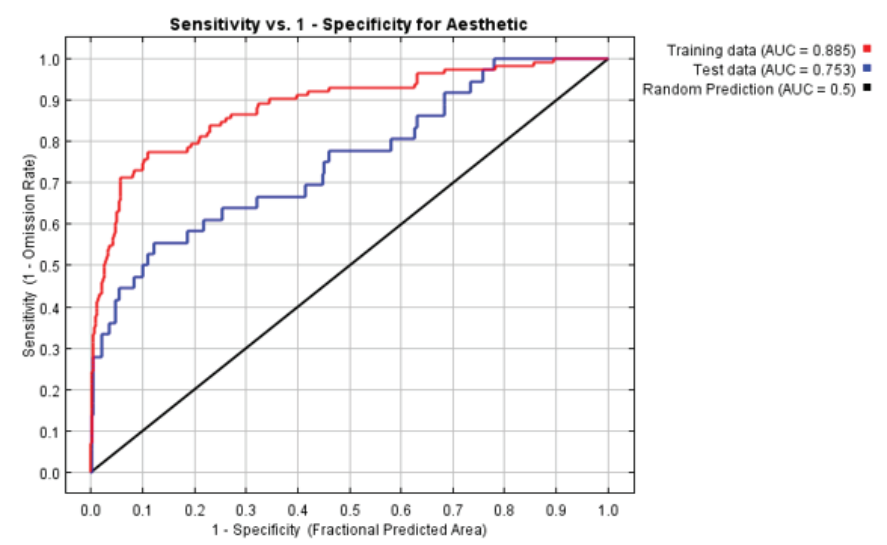

Figure 81. Example of Receiver Operating Characteristic curve and associated Area Under the Curve statistics generated by Maxent.

Models with AUC values of 0.5 or less perform at the level of random prediction (Phillips and others, 2006) or worse. Models with AUC values beginning at 0.70 (Swets, 1988; Hosmer and Lemeshow, 2000) to 0.75 (Elith and others, 2006) and above are considered potentially useful. SolVES instructs Maxent to withhold 25 percent of the points from each user-selected social-value type or survey subgroup as test points so that Maxent can calculate AUC statistics for training points (the remaining 75 percent) and test points. The training AUC indicates how well the model fits the primary study area, whereas the test AUC indicates the potential performance of the model in transferring social values to a similar area. When the View Results tool is used to generate map layouts of output generated by the Analyze Survey Data tool, the training AUC and test AUC statistics are reported at the bottom of the map layout, along with a brief statement interpreting the reported values.

Based on the test AUC statistic reported by the View Results tool, a user may wish to make adjustments to the model to potentially improve its social-value transfer performance. However, it should be kept in mind that higher test AUC values are not a guarantee of improved transfer performance (Sherrouse and Semmens, 2014). If necessary, users can adjust a model by changing the environmental variables it includes. One way to identify environmental variables that may be good candidates for removal is to examine the jackknife graphs of test AUC included in Maxent's HTML output (fig. 82). In the example below, the test AUC of the model could potentially be improved by removing dtr or slope from the model because the green bar indicating the test AUC of the model without the environmental variable extends beyond the red bar indicating the current test AUC. Users can complete such changes by setting up a new SolVES project and running the Analyze Survey Data tool without including the indicated environmental layers.

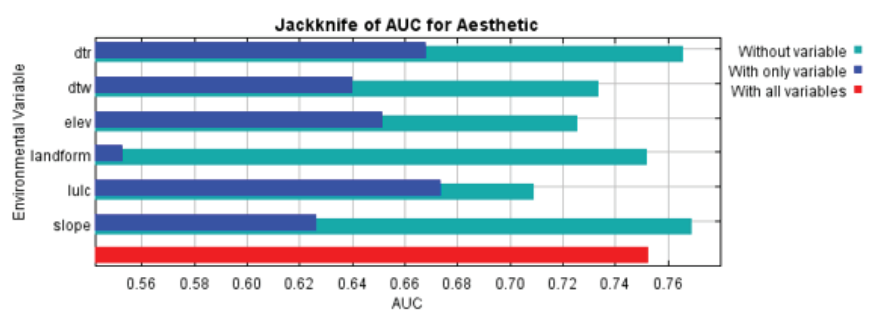

Figure 82. Example jackknife graph of test Area Under the Curve statistics generated by Maxent.

There is not a corresponding jackknife graph for training AUC; however, a user may wish to improve the goodness of fit of a social-value model to a primary study area instead of developing a more generalized, transferrable model. In this situation, a user can evaluate various environmental variable combinations by repeating analyses as separate SolVES projects. The percentage contribution and permutation importance of each environmental variable to a model (fig. 83) and the effect of each resulting variable combination on training AUC can be observed. Percent contribution is determined as a model is generated and can be impacted by the order in which the variables are evaluated. Variable contribution percentages should be interpreted cautiously if a model includes highly correlated variables. Permutation importance is calculated after a model is generated. 


\begin{tabular}{|r|r|r|}
\hline Variable & Percent contribution & Permutation importance \\
\hline \hline $\mathrm{elev}$ & 41.2 & 40.4 \\
\hline \hline $\mathrm{dtr}$ & 33.5 & 39.9 \\
\hline \hline $\mathrm{lulc}$ & 12.1 & 4 \\
\hline \hline $\mathrm{dtw}$ & 11 & 13.1 \\
\hline \hline $\mathrm{slope}$ & 1.7 & 2.6 \\
\hline landform & 0.5 & 0 \\
\hline
\end{tabular}

Figure 83. Example of variable contributions included in Maxent's HyperText Markup Language output.

\section{Digitizing Points Mapped by Survey Respondents}

Survey data collected using paper maps (unlike spatial data collected using online surveys) will require the use of a GIS to digitize the points mapped by survey respondents. Adequately designed public value and preference surveys include instructions directing respondents on how to mark and annotate points on the provided maps. If a respondent properly follows instructions like those described in the "Survey Data" section of appendix 1, there will be a point on the map notated with an indicator of its corresponding value type (for example, an "A" for aesthetic value) and a place name describing the location of the point. This point would be digitized at the location marked by the survey respondent, and all attributes of that point (SURVEY ID, VALUE ID, PLACE NAME) would be entered into the "SURVEY POINTS" attribute table accordingly. See the "Source Database Structure and Contents" section for additional information regarding data formatting.

Individual survey respondents may interpret survey instructions differently, so some general rules should be followed to avoid ambiguity by consistently guiding the digitization of each point. The following examples illustrate various ways in which survey respondents might deviate from instructions and the recommended approaches for attempting to preserve the intent of the respondent and to obtain data that are in a quantity and format useful for SolVES analyses:

- If a respondent indicates a location on the map with an annotation such as an " $A$ " for aesthetic value or indicates a specific place name but neglects to mark an actual point, digitize a point at the centroid of the annotation characters.

- If a respondent marks an area on the map using a circle or a polygon instead of a point, digitize a point at the centroid of the marked area.

- If a respondent marks a location on the map with a line segment representing a linear feature, digitize a point at the approximate middle of the line segment.
- If a respondent marks a single point on the map but indicates through annotation that it represents more than one value type, digitize a separate point for each indicated value type and attribute each point accordingly (for example, if a single point is annotated to indicate three value types, then digitize and attribute three points at that location).

- If a respondent marks a point on the map without indicating the value type it represents, the point should not be digitized (and if it is digitized, the point would not be included in any analyses).

- If a respondent indicates a value type and place name somewhere on the survey other than a location on the map without marking a corresponding point on the map itself, no point is digitized. (If a specific place name is unambiguous, can be resolved to a single point on the map, and has also been identified as representing a specific value type, it may be acceptable to digitize a point representing that location).

- If a respondent only indicates that they generally value the study area for all value types without providing any other information, no points are digitized.

Despite these recommended approaches to digitization, methodologies can vary depending on the skill, experience, or specific objectives of the individual(s) who complete(s) the digitization process. These variations should be kept in mind if relying on survey data digitized by other individuals or groups.

\section{Advanced Options}

\section{Recoding the "ATTITUDE_TYPES" Table}

SolVES 4.0 supports, by default, survey responses using a five-point Likert scale of attitudes ranging from strongly favor to strongly oppose as described in the "Survey Data" section of appendix 1. Survey data capturing a larger range of Likert scale values or different attitude descriptions can also be supported by modifying the "ATTITUDE_TYPES" table contents; however, even with such modifications, SolVES 4.0 will only support the segmentation of data by attitude type into two groups. This is controlled by the two available options available through the Analyze Survey Data tool's "Attitude or Preference" drop-down box and the "ATTITUDE_CODE" to which they correspond ("FAVOR" means favor or strongly favor, and "OPPOSE" means oppose or strongly oppose). Any modifications must conform to this dichotomous pattern.

The recoding example illustrated in figure 84 presents a change in the number of Likert scale values (from 5 to 7) and what the values represent (relative agreement instead of favorability) to match the corresponding response data the user would have loaded into the "USE_ATTITUDE" table. In this example, if "Favor or Strongly Favor" is selected with 


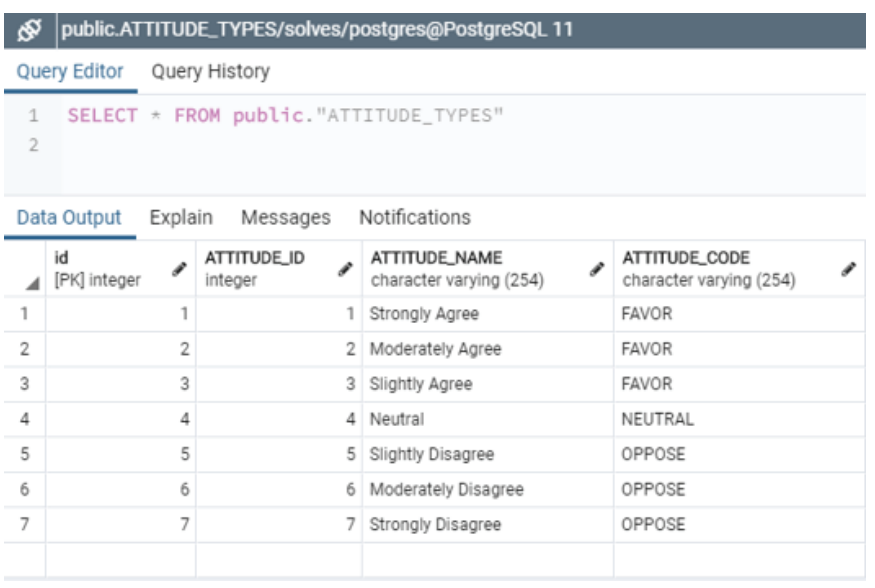

Figure 84. Example of a recoded "ATTITUDE_TYPES" table as viewed with pgAdmin 4.

the Analyze Survey Data tool, records from the "USE_ATTITUDE" table having "ATTITUDE_ID" values of 1, 2, or 3 (ATTITUDE_CODE=FAVOR) will be selected. If "Oppose or Strongly Oppose" is selected, records with an ATTITUDE_ID of 5, 6, or 7 (ATTITUDE_CODE=OPPOSE) will be selected. In all situations, any response data coded as "NEUTRAL" will not be included in the analysis. Modifications could also be made to support scales having less than five points. In all situations, the "ATTITUDE_NAME" field can be used to enter descriptions that will allow users to track the actual meaning of their survey response data versus the limited values supported by the user interface.

\section{Substituting the Public Use Parameter}

SolVES is designed to accept a public use parameter for defining survey subgroups along with the attitude or preference parameter; however, if a user has survey results that would be useful for defining survey subgroups in a manner other than with public uses (for example, attitudes or preferences regarding particular issues impacting a study area such as fire management or species conservation), they may do so as long as the attitude or preference responses are structured in the same format as indicated in table 1.1 in appendix 1 or can be supported by adjustments to ATTITUDE_TYPES as described in the "Recoding the ATTITUDE_TYPES Table" section. Assuming the response structure is suitable for SolVES to support through the "USE_ATTITUDE" and "ATTITUDE_TYPES" tables, users may load their parameter data into the "USE_TYPES" table in the source database as a substitute for public use. Be aware that the SolVES user interface will still refer to the parameter as public use, but this will have no effect on the analysis.

\section{Creating Survey Data Subgroups Before Loading SolVES}

The SolVES Analyze Survey Data tool only accepts public use (or user-provided substitutes) and attitude or preference parameters to define subgroups; however, users may wish to subdivide their survey data in some manner, such as by certain demographic characteristics like age or income. This can be accomplished by creating separate datasets for each subgroup, loading them into separate source databases for analysis, and leaving the public use and attitude or preference parameters blank to process all survey data within each database. Users, of course, will need to track and manage the results because SolVES will not recognize or meaningfully label the results as belonging to a specific subgroup. Users should also be aware that overly restrictive criteria may result in subgroups that are too small for SolVES to analyze for any meaningful results.

\section{Sharing Models for Value Transfer}

At some point, users may wish to share a social-value model they have generated with others who would like to apply the model in a similar study area using the Transfer Values tool. Although the lambdas file generated by Maxent contains the actual model that other SolVES users would apply to environmental layers for their own study area, additional metadata describing the content and format of the required environmental layers and other model parameters are necessary. To apply a model using the Transfer Values tool, users will require the following information:

- The model's output cell size.

- The maximum value the model attained on the value index.

- For continuous data, users will need a description of the value range and units.

- For categorical data, users will need the numerical and textual description of each value type.

- The source of the environmental data used to generate the model may also assist other users in finding the environmental layers necessary to apply the model to their own study area.

- The model's test AUC as an indicator of its potential performance in transferring social values to similar study areas.

- Copies of the environmental data graphs generated by SolVES so other users can observe the relations between the environmental variables and the value index. 
- Where possible, the metadata should include some description of the social-value model's social context such as brief demographic profile of the respondents whose data were used to generate the model.

As a guide to metadata format and contents, see the metadata template included in the $\mid$ SolVES $\backslash$ Doc folder and the sample model metadata included as HTML files in $\backslash$ SolVES $\backslash$ Models as part of the SolVES 4.0 tool download. Completed metadata files should be placed in the same models subfolder as the corresponding lambdas file (for example, |SolVES $\backslash$ Models $\backslash$ Pike and San Isabel National Forest $\backslash A$ ll Surveys $\backslash$ Aesthetic.html).

\section{Troubleshooting Common Errors}

\section{Unable to Install PostGIS}

If the warning and error messages shown in figure 85 are encountered when Stack Builder attempts to install PostGIS after the PostgreSQL installation, PostgreSQL should be uninstalled and then reinstalled according to the "PostgreSQL Installation" section; however, the Stack Builder component should not be selected (see fig. 6). Once the PostgreSQL has reinstalled, navigate to the $\backslash$ SolVES $\backslash$ Install $\backslash$ PostgreSQL folder and select the "postgis-bundle-pg11x64-setup-2.5.3-1" installation file (fig. 86). Continue with the PostGIS installation as described in the PostgreSQL installation section.

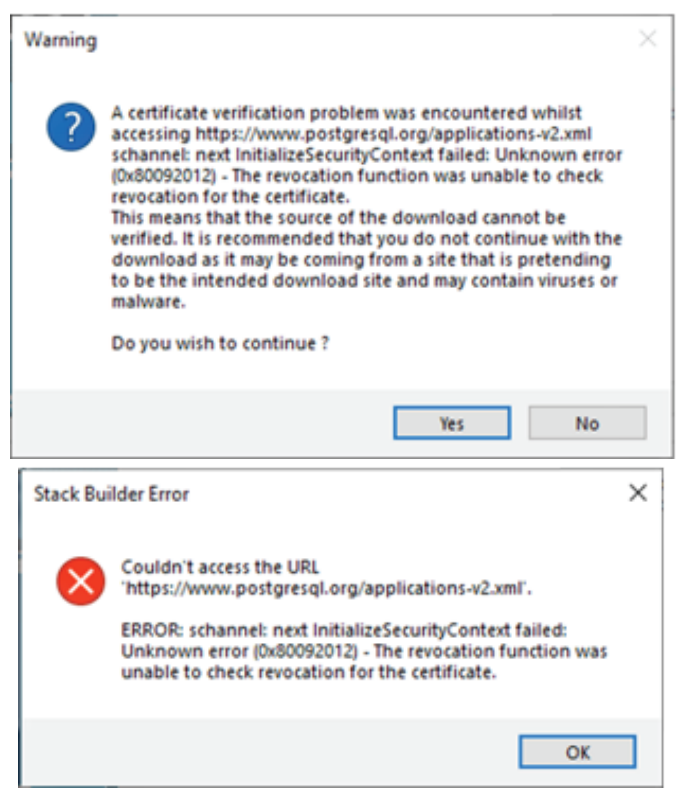

Figure 85. Warning and error messages received during PostGIS 2.5.3 installation.

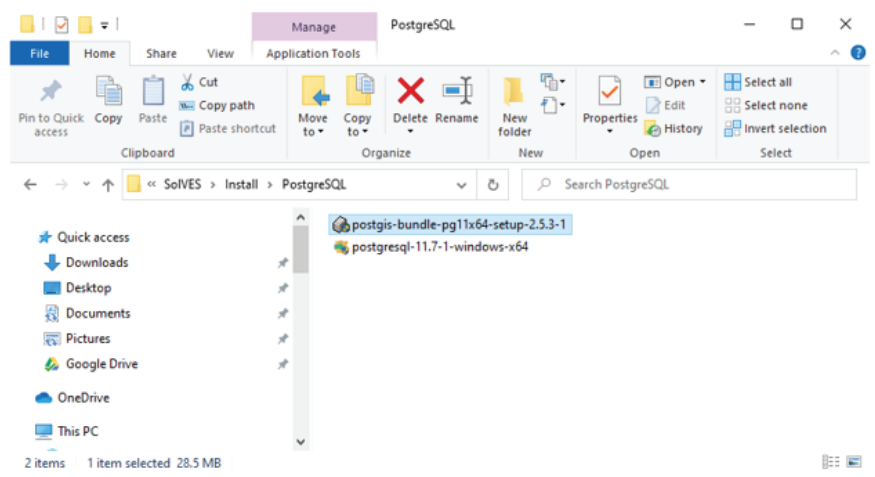

Figure 86. Location of the PostGIS 2.5.3 installation file.

\section{Unable to Access Python}

If the option to install the SolVES plugin from "solves. zip" is unavailable, refer to the "Confirming QGIS Settings for SolVES 4.0" section and confirm that the path to the Python directory is included in the PATH environment variable. Python upgrades made by system administrators could lead to a change in the name of the Python directory, which may require the PATH environment variable to be updated in QGIS.

\section{Unable to Access Java}

If runtime errors occur referencing the inability of SolVES to locate "java" or "javaw," refer to the "Confirming QGIS Settings for SolVES 4.0" section to make sure that the path to the Java directory is included in the PATH environment variable. Java upgrades made by system administrators could lead to a change in the name of the Java directory, which may require the PATH environment variable to be updated in QGIS.

\section{Invalid Features}

If an error occurs referencing invalid features (usually associated with the calculation of statistics for the environmental data layers), refer to the "Confirming QGIS Settings for SolVES 4.0" section to make sure that the invalid features filtering setting has been set to "Do not filter (better performance)."

\section{Environmental Data Not Found}

If an error is returned from Maxent indicating a selected environmental layer was not found, use pgAdmin 4 to view the contents of the "ENV_LAYERS" table and confirm that all environmental layers loaded in the solves database are listed in the table and that the names in the table are identical to the layer names. 


\section{Missing Study Area Layer}

If a "_core.QgsProcessingException: Unable to execute algorithm Incorrect parameter value for CRS" error occurs, then the "STUDY AREA" layer is either missing from the solves database, has not been named correctly, or has not been properly projected. Make any necessary corrections to the "STUDY_AREA" layer and reload it to the solves database.

\section{Missing Survey Points Layer}

If a "_core.QgsProcessingException: There were errors executing the algorithm" error occurs, it is likely that the "SURVEY POINTS" layer is either missing from the solves database or has not been named correctly. Make any necessary corrections to the "SURVEY_POINTS" layer and reload it to the solves database. If this error is encountered, but the "SURVEY_POINTS" layer is found to not be the source, review the "PostgreSQL Source Database Structure and Contents" section to confirm that all required data layers are provided and properly formatted in the solves database.

\section{No Results Data}

If a "TypeError: 'NoneType' object is not iterable" error occurs when attempting to use the View Results tool, then no project results were found in the results directory.

\section{Incorrect Regional Settings}

If errors are encountered that are not described by any of the other errors outlined in this section, refer to the "Confirming Windows Regional Settings" section to make sure all settings are correct.

\section{Incorrect Text Size}

If the SolVES 4.0 user-interface text appears too large, access the Windows display settings and set the scale and layout to " $100 \%$." This setting can be changed back to the original value when not using SolVES 4.0.

\section{Troubleshooting Assistance}

If problems with installing or running SolVES 4.0 persist after following the troubleshooting assistance provided in the user manual, contact gs-solves_support@usgs.gov for technical support.

\section{References Cited}

Alessa, L.N., Kliskey, A.A., and Brown, G., 2008, Socialecological hotspots mapping-A spatial approach for identifying coupled social-ecological space: Landscape and Urban Planning, v. 85, no. 1, p. 27-39. [Also available at https://doi.org/10.1016/j.landurbplan.2007.09.007.]

Brown, G., 2004, Mapping spatial attributes in survey research for natural resource management-Methods and applications: Society \& Natural Resources, v. 18, no. 1, p. 17-39. [Also available at https://doi.org/10.1080/ 08941920590881853.]

Brown, G., and Alessa, L., 2005, A GIS-based inductive study of wilderness values: International Journal of Wilderness, v. 11, no. 1, p. $14-18$.

Brown, G., and Reed, P., 2000, Validation of a forest values typology for use in national forest planning: Forest Science, v. 46 , no. 2 , p. $240-247$.

Brown, G.G., Reed, P., and Harris, C.C., 2002, Testing a placebased theory for environmental evaluation-An Alaska case study: Applied Geography (Sevenoaks, England), v. 22, no. 1, p. 49-76. [Also available at https://doi.org/10.1016/ S0143-6228(01)00019-4.]

Brown, G., Smith, C., Alessa, L., and Kliskey, A., 2004, A comparison of perceptions of biological value with scientific assessment of biological importance: Applied Geography (Sevenoaks, England), v. 24, no. 2, p. 161-180. [Also available at https://doi.org/10.1016/j.apgeog.2004.03.006.]

Clement, J.M., 2006, Spatially explicit values on the Pike and San Isabel National Forests in Colorado: Fort Collins, Colorado State University, Ph.D. dissertation, ProQuest/ UMI, AAT 3246268.

Costanza, R., d'Arge, R., de Groot, R., Farber, S., Grasso, M., Hannon, B., Limburg, K., Naeem, S., O’Neill, R.V., Paruelo, J., Raskin, R.G., Sutton, P., and van den Belt, M., 1997, The value of the world's ecosystem services and natural capital: Nature, v. 387, no. 6630, p. 253-260. [Also available at https://doi.org/10.1038/387253a0.]

Cowling, R.M., Egoh, B., Knight, A.T., O’Farrell, P.J., Reyers, B., Rouget, M., Roux, D.J., Welz, A., and WilhelmRechman, A., 2008, An operational model for mainstreaming ecosystem services for implementation: Proceedings of the National Academy of Sciences of the United States of America, v. 105, no. 28, p. 9483-9488. [Also available at https://doi.org/10.1073/pnas.0706559105.]

Daily, G.C., 1997, Introduction-What are ecosystem services? in Daily, G.C., ed., Nature's services - Societal dependence on natural ecosystems: Washington, D.C., Island Press, p. 1-10. 
Elith, J., Graham, C.H., Anderson, R.P., Dudík, M., Ferrier, S., Guisan, A., Hijmans, R.J., Huettmann, F., Leathwick, J.R., Lehmann, A., Li, J., Lohmann, L.G., Loiselle, B.A., Manion, G., Moritz, C., Nakamura, M., Nakazawa, Y., Overton, J.M.M., Townsend Peterson, A., Phillips, S.J., Richardson, K., Scachetti-Pereira, R., Schapire, R.E., Soberón, J., Williams, S., Wisz, M.S., and Zimmermann, N.E., 2006, Novel methods improve prediction of species' distributions from occurrence data: Ecography, v. 29, no. 2, p. 129-151. [Also available at https://doi.org/10.1111/ j.2006.0906-7590.04596.x.]

Elith, J., Phillips, S.J., Hastie, T., Dudík, M., Chee, Y.E., and Yates, C.J., 2010, A statistical explanation of MaxEnt for ecologists: Diversity \& Distributions, v. 17, no. 1, p. 1-15.

Hosmer, D.W., and Lemeshow, S., 2000, Applied logistical regression 2nd ed.: Hoboken, N.J., John Wiley \& Sons, 392 p. [Also available at https://doi.org/10.1002/ 0471722146.]

Kumar, M., and Kumar, P., 2008, Valuation of the ecosystem services - A psycho-cultural perspective: Ecological Economics, v. 64, no. 4, p. 808-819. [Also available at https://doi.org/10.1016/j.ecolecon.2007.05.008.]

Maleki, S., 2008, Counting all that matters-Recognizing the value of ecosystem services: Portland, Oreg., U.S. Department of Agriculture, Forest Service, Pacific Northwest Research Station, Science Update 16, 12 p.

Millennium Ecosystem Assessment, 2003, Ecosystems and human well-being-A framework for assessment: Washington, D.C., Island Press, 245 p.

Nijkamp, P., Vindigni, G., and Nunes, P.A.L.D., 2008, Economic valuation of biodiversity-A comparative study: Ecological Economics, v. 67, no. 2, p. 217-231. [Also available at https://doi.org/10.1016/j.ecolecon.2008.03.003.]

Phillips, S.J., Anderson, R.P., Dudík, M., Schapire, R.E., and Blair, M.E., 2017, Opening the black box-An open-source release of Maxent: Ecography, v. 40, no. 7, p. 887-893. [Also available at https://doi.org/10.1111/ecog.03049.]

Phillips, S.J., Anderson, R.P., and Schapire, R.E., 2006, Maximum entropy modeling of species geographic distributions: Ecological Modelling, v. 190, no. 3-4, p. 231-259. [Also available at https://doi.org/10.1016/ j.ecolmodel.2005.03.026.]

Phillips, S.J., and Dudík, M., 2008, Modeling of species distributions with Maxent-New extensions and a comprehensive evaluation: Ecography, v. 31, no. 2, p. 161-175. [Also available at https://doi.org/10.1111/j.09067590.2008.5203.x.]
Phillips, S.J., Dudík, M., and Schapire, R.E., 2004, A maximum entropy approach to species distribution modeling, in Greiner, R., and Schuurmans, D., eds., International conference on machine learning, 21st, ACM Press, 2004, Proceedings: Banff, Alberta, Canada, International Machine Learning Society, p. 655-662.

Reed, P., and Brown, G., 2003, Values suitability analysis-A methodology for identifying and integrating public perceptions of ecosystem values in forest planning: Journal of Environmental Planning and Management, v. 46, no. 5, p. 643-658. [Also available at https://doi.org/10.1080/ 0964056032000138418.]

Semmens, D.J., Sherrouse, B.C., and Ancona, Z.H., 2019, Using social-context matching to improve spatial functiontransfer performance for cultural ecosystem service models: Ecosystem Services, v. 38, p. 100945. [Also available at https://doi.org/10.1016/j.ecoser.2019.100945.]

Sherrouse, B.C., Clement, J.M., and Semmens, D.J., 2011, A GIS application for assessing, mapping, and quantifying the social values of ecosystem services: Applied Geography (Sevenoaks, England), v. 31, no. 2, p. 748-760. [Also available at https://doi.org/10.1016/j.apgeog.2010.08.002.]

Sherrouse, B.C., and Semmens, D.J., 2014, Validating a method for transferring social values of ecosystem services between public lands in the Rocky Mountain region: Ecosystem Services, v. 8, p. 166-177. [Also available at https://doi.org/10.1016/j.ecoser.2014.03.008.]

Sherrouse, B.C., and Semmens, D.J., 2020, SolVES 4.0: U.S. Geological Survey software release, https:/doi.org/10.5066/ P9URDZ4V.

Sherrouse, B.C., Semmens, D.J., Ancona, Z.H., and Brunner, N.M., 2017, Analyzing land-use change scenarios for trade-offs among cultural ecosystem services in the Southern Rocky Mountains: Ecosystem Services, v. 26, p. 431-444. [Also available at https://doi.org/10.1016/ j.ecoser.2017.02.003.]

Sherrouse, B.C., Semmens, D.J., and Clement, J.M., 2014, An application of Social Values for Ecosystem Services (SolVES) to three national forests in Colorado and Wyoming: Ecological Indicators, v. 36, p. 68-79. [Also available at https://doi.org/10.1016/j.ecolind.2013.07.008.]

Silverman, B.W., 1986, Density estimation for statistics and data analysis: New York, Chapman and Hall, 176 p. [Also available at https://doi.org/10.1007/978-1-4899-3324-9.]

Swets, J.A., 1988, Measuring the accuracy of diagnostic systems: Science, v. 240, no. 4857, p. 1285-1293. [Also available at https://doi.org/10.1126/science.3287615.] 



\section{Appendix 1. Social Values for Ecosystem Services, Version 4.0, Data Requirements, Structure, and Management}

\section{Data Requirements}

Social Values for Ecosystem Services (SolVES) uses various geospatial and tabular data, which may be obtained by users in a variety of formats (for example, shapefiles, Tagged Image File Format [TIF], comma-separated values [CSV]). For use with SolVES, these data must be imported into the required format of the SolVES source database, "solves." The rest of this appendix describes the required data.

\section{Environmental Data}

SolVES requires only one environmental data layer to run; however, for practical application, users may include any number and type of continuous and categorical environmental data in their analyses. All environmental data layers must be raster based and share a common projection and extent.

\section{Survey Data}

Specifically formatted value and preference survey response data are required to use the Ecosystem Services Social-Values Model and Value Mapping Model. These data are not required, however, if only using the Value Transfer Mapping Model. The formats of these data are based on value and preference survey results obtained from J.M. Clement (written commun., 2008) for the Pike and San Isabel National Forests in Colorado that, in turn, are based on data collection procedures described by Brown and others (2002). A sample of the survey document is available in the $\backslash$ SolVES $\backslash$ Doc subfolder.

SolVES was initially developed from responses to a random mail survey of 2,000 households within 45 miles of the Pike and San Isabel National Forests performed in late 2004 and early 2005 (J.M. Clement, written commun., 2008). The response rate was about 33 percent with 684 surveys returned. The survey was divided into five sections. Section 1 requested information regarding each respondent's familiarity with the Pike and San Isabel National Forests, such as when and how often they visited, if they derived any income from the Pike and San Isabel National Forests and their interest level in what happens to the Pike and San Isabel National Forests in the next 10 to 15 years. Using a five-point Likert scale ranging from strongly favor to strongly oppose, section 2 requested respondents to indicate their attitudes regarding 18 public uses of the Pike and San Isabel National Forests such as logging for wood products, motorized recreation, and wilderness. Section 3 allowed respondents to indicate their views regarding various issues impacting the Pike and San Isabel National Forests, such as the extent and purpose of road building and logging, reservoir development, and tradeoffs between recreational use and environmental quality. The first part of section 4 (4A) requested that respondents allocate or "spend" $\$ 100$ among 12 social-value types such as aesthetics, biodiversity, and recreation associated with the Pike and San Isabel National Forests. Although dollar units were used for convenience to express value denominations (for example, points could have been used instead of dollars), the survey instructions included an explanation that this was not a reference to any actual money held by the respondent or the U.S. Department of Agriculture Forest Service. When the allocation exercise was completed, respondents were then instructed in the second part of section 4 (4B) to hand-mark points (later digitized into a geographic data layer) on a series of maps of the Pike and San Isabel National Forests corresponding to the social-value types to which they had allocated dollars. If the respondent had allocated dollars to aesthetic value, for example, they were to place a point on the map at as many as four locations indicating aesthetic, label and number each mark accordingly, and provide a place name describing the location of the point. Of the 684 surveys returned, the mapping section was completed for 55 percent of them. Finally, section 5 of the survey requested various demographic and socioeconomic information from each respondent. Further details regarding the Pike and San Isabel National Forests survey are provided in Sherrouse and others (2014).

The survey data used by SolVES are based on survey sections $2,4 \mathrm{~A}$, and $4 \mathrm{~B}$; however, the only survey data required to use SolVES are the point data from survey section 4B. To analyze user-supplied survey data with SolVES, the structural requirements set forth in the remainder of this section must be met. Surveys designed to collect data containing the specific elements described below, even if not otherwise designed like the Pike and San Isabel National Forests survey, can be used with SolVES.

Users providing their own survey data must assign a unique identifier (SURVEY_ID), an integer value, to each survey in order to distinguish each survey and to relate its separate sections. See the "PostgreSQL Source Database Structure and Contents" section for additional details regarding the location of "SURVEY ID" within the database schema.

"USE_ATTITUDE" (based on survey section 2).-

SolVES supports public use data that include responses as to whether such uses are favored or opposed. Based on the Likert scale used in the original Pike and San Isabel National Forests survey, these responses are structured as an integer range from 
1 to 5 by default (table 1.1). However, the allowable values can be modified as described in the "Recoding the ATTITUDE_TYPES Table" subsection of the "Advanced Options" section. For information regarding how parameters other than public uses might be used by SolVES, see the "Substituting the Public Use Parameter" subsection of the "Advanced Options" section. Please note the "USE_ATTITUDE" table can be left unpopulated if these data were not collected or if a user wishes to only analyze data from all surveys without distinguishing any survey subgroups. Please note that the "By Social Value Type Across Survey Subgroups" value comparison type option will not function if "USE_ATTITUDE" data are not provided.

"VALUE_ALLOCATION" (based on survey section 4A).- - SolVES supports numeric values allocated among the survey's social-value types to be used as weights; however, value allocations are not required to run SolVES.

"SURVEY_POINTS" (based on survey section 4B).SolVES supports point layers representing the points that survey respondents marked on a map or maps of a study area. Each point must be identified as representing one of the social-value types included in the survey. It must share a common projection with all environmental data layers and study area boundary. See the "Notes Regarding the Digitization of Points Mapped by Survey Respondents" section for additional information.

\section{Other Spatial Data}

SolVES requires a "STUDY_AREA" polygon feature representing the study area boundary. It must share a common projection with all environmental data layers and survey points. This feature may exist as multiple parts, but it must be defined as a single feature. One way to confirm this is to view the attribute table for the "STUDY_AREA" feature class to ensure it contains only one record. This is necessary to prevent errors with the buffering option.

\section{Data Management}

\section{Directory Structure}

SolVES is the root directory containing the folders described below (table 1.2).

Table 1.1. Default Likert scale attitude or preference integer values used by Social Values for Ecosystem Services, version 4.0.

\begin{tabular}{lll}
\hline & Attitude or preference & Integer value \\
\hline Strongly favor & 1 \\
Favor & 2 \\
Neutral & 3 \\
Oppose & 4 \\
Strongly oppose & 5 \\
\hline
\end{tabular}

Table 1.2. Description of the Social Values for Ecosystem Services, version 4.0, directory contents.

[SolVES, Social Values for Ecosystem Services]

\begin{tabular}{ll}
\hline \multicolumn{1}{c}{ Folder name } & \multicolumn{1}{c}{ Folder description } \\
\hline Data & $\begin{array}{c}\text { Database backup files for the "solves" database. These include two files containing sample data for dem- } \\
\text { onstration purposes and one file that can be populated with user-supplied data for their own analyses. } \\
\text { SolVES readme file and metadata template. }\end{array}$ \\
Doc & QGIS, PostgreSQL, and SolVES plugin installation files. \\
Laystall & Files required to generate SolVES map layouts. \\
Maxent & Maxent maximum entropy modeling software. \\
Models & Maxent lambdas files for use with the SolVES Transfer Values tool. \\
Results & Final data generated by SolVES. \\
Scratch & Intermediate data generated by SolVES. \\
\hline
\end{tabular}




\section{PostgreSQL Source Database Structure and Contents}

A schematic of the solves database structure is shown below (fig. 1.1). The diagram summarizes the contents of a fully loaded database and the relations between each survey data element. "SURVEY_ID" does not exist as a distinct entity in the database; instead, it is a field included in the attribute table of each of the related elements. The field is included in the schematic to emphasize its role as a primary key for relating the data. The "(1-1)" symbology indicates a one-to-one relation, whereas the "(1-M)" symbology indicates a one-tomany relation between elements. For example, one record in "ENV_LAYERS" is related to only one environmental layer, but one survey (SURVEY_ID) may be related to many survey points (SURVEY_POINTS). Additional descriptions of the database contents are included in the remainder of this section.

Environmental Layers (blue, fig. 1.1).-These layers are supplied by the user and may range from one to any number of raster datasets.

Tables (yellow, fig. 1.1).- These tables are present when SolVES 4.0 is downloaded. The default contents of the "ATTITUDE_TYPES" table are already loaded, but users must load their own survey data into the remaining tables. Individual table structures are described next to serve as guides to users for preparing and properly formatting their survey data (table 1.3). The "ENV_LAYERS" table is new in SolVES 4.0. It requires users to provide the name of each raster loaded into the database exactly as the raster was named. Users must then indicate if each raster includes continuous or categorical data by entering a " 0 " for continuous data or a " 1 " for categorical data in the "VAR_TYPE" field. SolVES 4.0 uses these data to determine the data type of each raster, which eliminates the need for users to enter this information for every SolVES analysis. An example of a populated "ENV_LAYERS" table is shown in figure 1.2.

Vector Layers (green, fig. 1.1).-These data include the mapped points along with polygon layers representing the study area and other boundaries as listed in table 1.4. Users must supply their own "SURVEY_POINTS" and "STUDY AREA" layers. States and counties are included in the SolVES 4.0 download and are available to use for generating a locator map that assists in orienting the study area within a broader geographic area. For study areas outside the contiguous 48 States, we recommend that users supply an appropriate polygon layer for generating the locator map; however, this is not required.

Any projected coordinate system suitable for the study area may be used; however, the projection should have linear units defined in meters. All spatial data must share a common projection and all environmental data layers must have identical extents before completing any SolVES analyses.

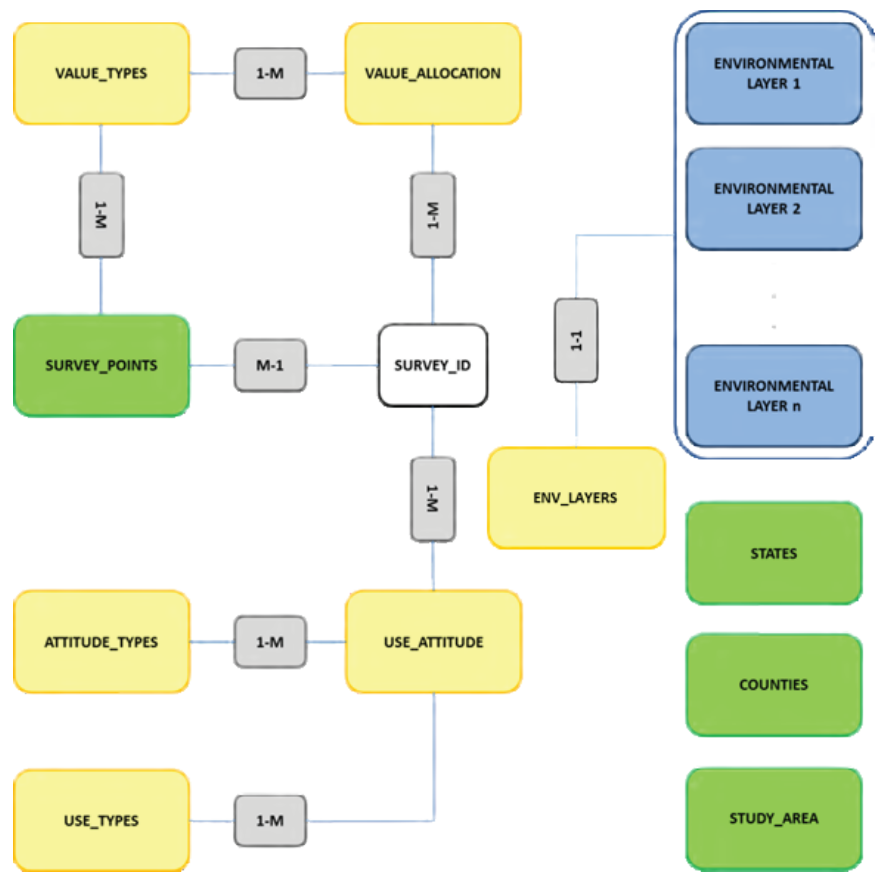

Figure 1.1. Schematic of the "solves" database in PostgreSOL 11.7. 
Table 1.3. Table formatting requirements for the "solves" database in PostgreSOL 11.7.

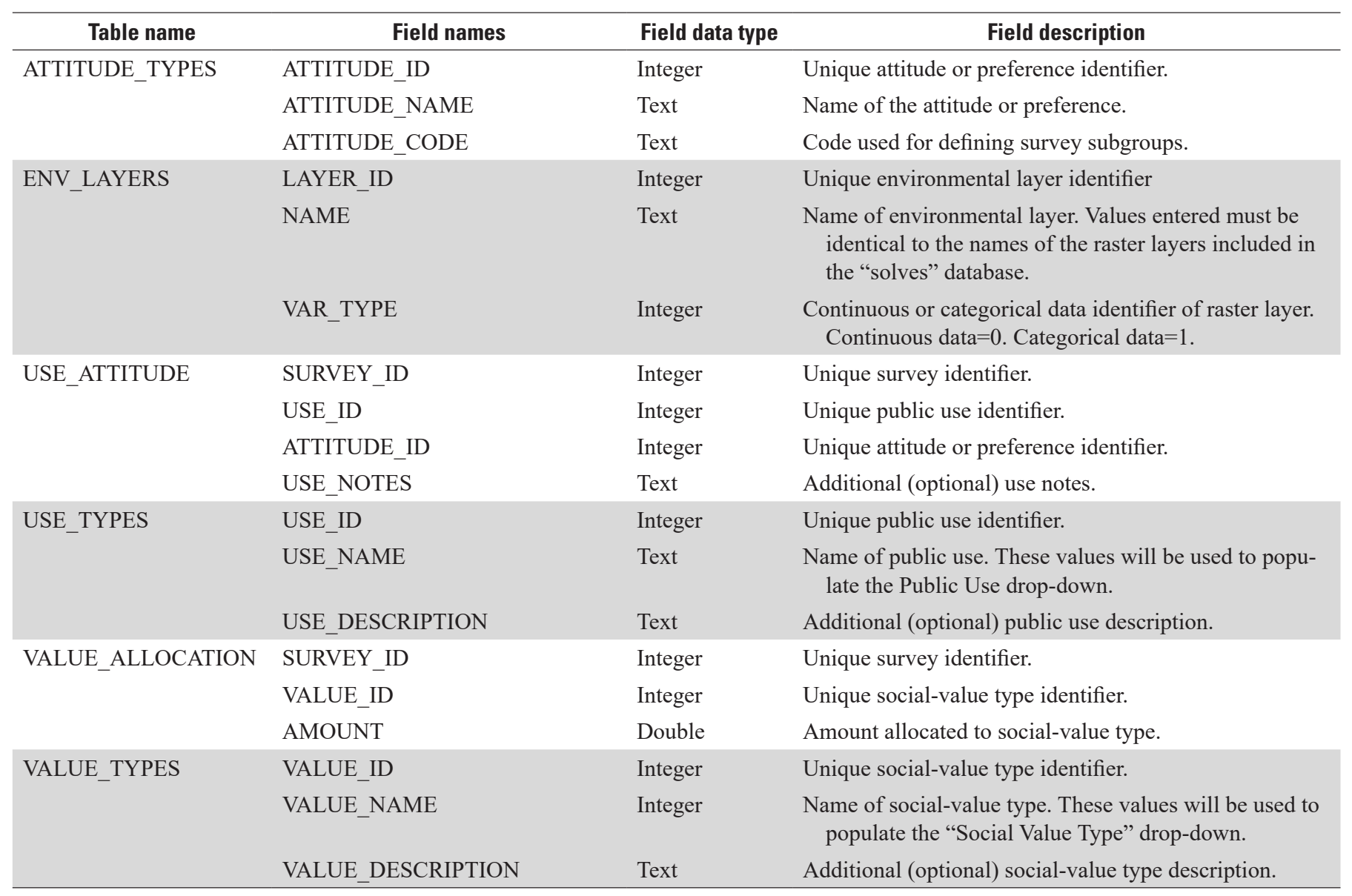

\begin{tabular}{|c|c|c|c|c|c|c|c|c|}
\hline \multicolumn{2}{|c|}{ Data Output } & Explain & \multicolumn{2}{|c|}{ Messages } & Notifications & \multirow[b]{2}{*}{ 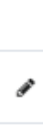 } & \multirow[b]{2}{*}{$\begin{array}{l}\text { VAR_TYPE } \\
\text { integer }\end{array}$} & \multirow[b]{2}{*}{ 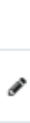 } \\
\hline$\Delta$ & $\begin{array}{l}\text { id } \\
{[\mathrm{PK}] \text { integer }}\end{array}$ & 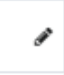 & $\begin{array}{l}\text { LAYER_ID } \\
\text { integer }\end{array}$ & 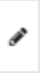 & $\begin{array}{l}\text { NAME } \\
\text { character varying }\end{array}$ & & & \\
\hline 1 & & 1 & & 1 & DTR & & & 0 \\
\hline 2 & & 2 & & 2 & DTW & & & 0 \\
\hline 3 & & 3 & & 3 & ELEV & & & 0 \\
\hline 4 & & 4 & & 4 & HILLSHADE & & & 1 \\
\hline 5 & & 5 & & 5 & LANDFORM & & & 1 \\
\hline 6 & & 6 & & 6 & LULC & & & 1 \\
\hline 7 & & 7 & & 7 & SLOPE & & & 0 \\
\hline
\end{tabular}

Figure 1.2. Example of a populated "ENV_LAYERS" table as viewed in pgAdmin 4.

Table 1.4. Vector layer formatting requirements for the "solves" database in PostgreSQL 11.7.

\begin{tabular}{llll}
\hline \multicolumn{1}{c}{ Vector layer name } & \multicolumn{1}{c}{ Field names } & \multicolumn{1}{c}{ Field data type } & \multicolumn{1}{c}{ Field description } \\
\hline SURVEY_POINTS & SURVEY_ID & Integer & Unique survey identifier. \\
& VALUE_ID & Integer & Unique social-value type identifier. \\
& PLACE_NAME & Text & Place name at location of point (optional). \\
STUDY_AREA & NAME & Text & Name of study area. \\
\hline
\end{tabular}




\section{Final Results and Intermediate Data}

\section{Output Structure}

During SolVES processing, project-level folders bearing the project name and analysis-level folders bearing the name of the selected survey subgroup ("All Surveys" if no survey subgroup is selected) or social-value type within the projectlevel folder are created within the results and scratch folders of the SolVES directory (fig. 1.3).

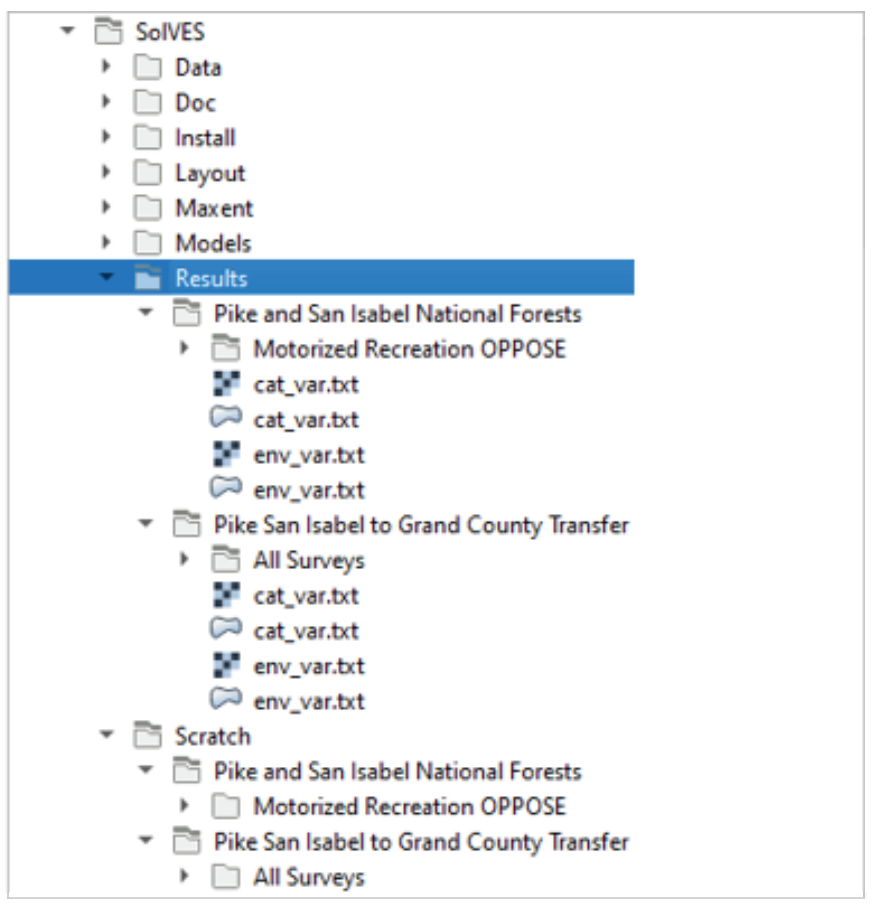

Figure 1.3. Example of project-level and analysis-level folders created under the results and scratch folders in the Social Values for Ecosystem Services, version 4.0, directory.

\section{Results Folder Contents}

SolVES and Maxent output are written to the results analysis-level folders (fig. 1.4). Final social-value maps are written to the rasters folder as TIF files. The study area boundary (STUDY_AREA_FINAL) and survey points (SURVEY POINTS_SELECT) used in the specific analysis are saved to the vectors folder as shapefiles. Environmental metric graphs generated for the final map layout are saved to the graphs folder as image files. The source data for the environmental metric graphs are available in the vectors folder under the corresponding social-value type or survey subgroup folder.
Summary tables of average nearest neighbor statistics and the maximum value index score attained by each social-value type or survey subgroup are written to the tables folder as CSV files. Log files for each SolVES run are written to the log folder. A "Maxent_Data" folder is also written to the results folder. It contains output from Maxent and specially formatted survey point and environmental data inputs for Maxent. The environmental folder contains resampled environmental

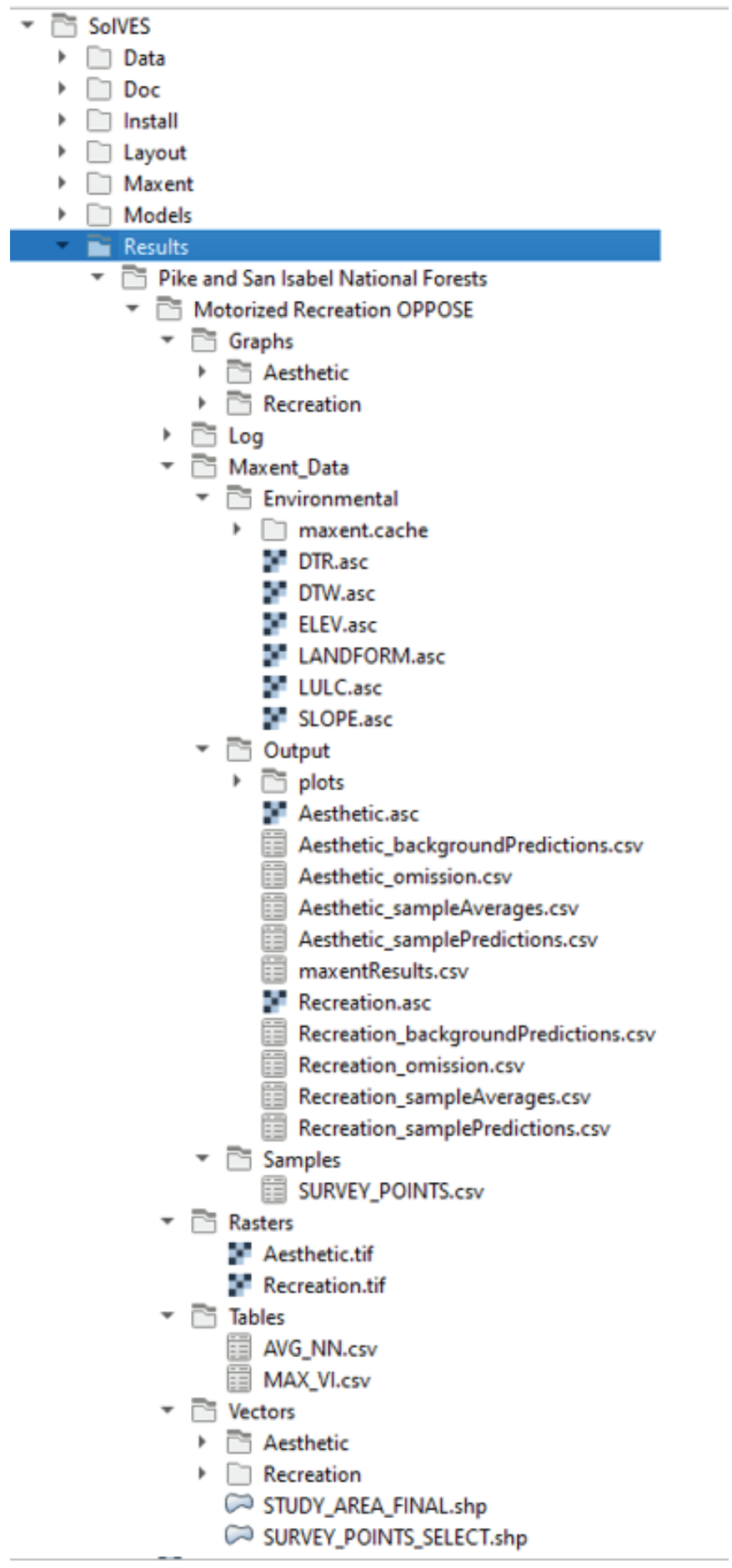

Figure 1.4. Results folder contents as seen in QGIS 2.5.3. 
layers from the solves database that have been converted to American Standard Code for Information Interchange (ASCII) format. The samples folder contains the user-selected survey points converted to CSV format. The output folder contains logistic value grids generated by Maxent as ASCII files, along with other files describing Maxent results. Detailed information regarding final outputs is included in appendix 3.

Accessing the Maxent_DatalOutput folder through Windows, users will also see Hypertext Markup Language (HTML) files associated with each model generated by Maxent that provide additional information regarding the Maxent results (including Area Under the Curve [AUC] statistics, jackknife graphs, variable contribution percentages), along with links to the other files contained in the output folder (fig. 1.5). See the "Interpreting and Adjusting Maxent's AUC Values and Variable Contributions" section for additional information regarding these HTML file contents.

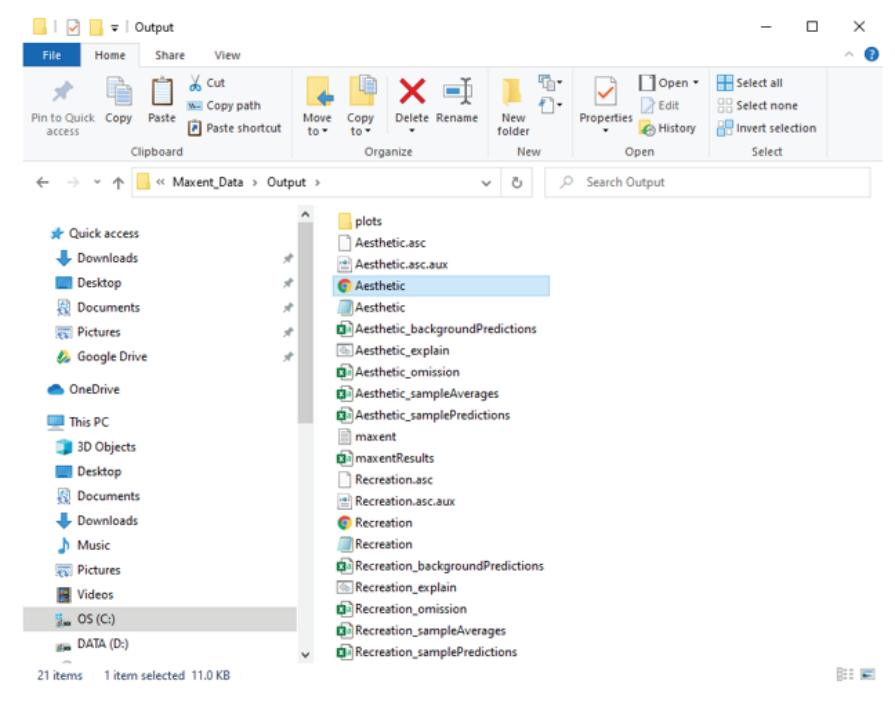

Figure 1.5. Maxent_DatalOutput folder contents as seen in Windows.

\section{Scratch Folder Contents}

The scratch folder contains all intermediate rasters, tables, and vector layers generated by SolVES (fig. 1.6). Detailed information describing intermediate outputs is included in appendix 3 .

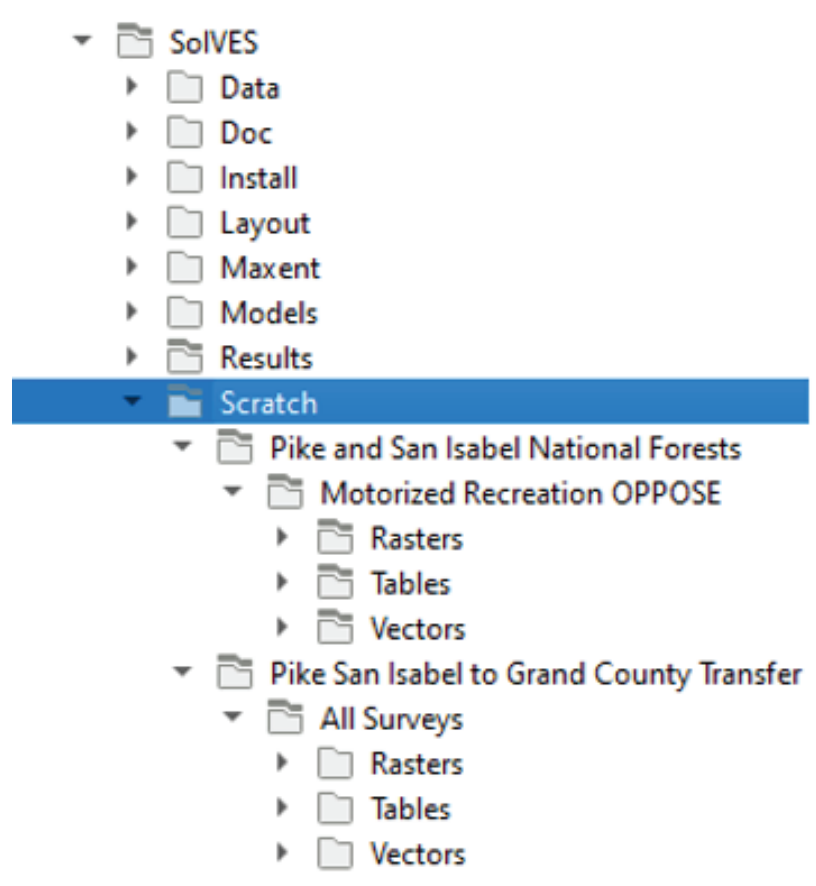

Figure 1.6. Scratch folder structure as seen in QGIS 3.8.2. 


\section{References Cited}

Brown, G.G., Reed, P., and Harris, C.C., 2002, Testing a placebased theory for environmental evaluation-An Alaska case study: Applied Geography (Sevenoaks, England), v. 22,

no. 1, p. 49-76. [Also available at https://doi.org/10.1016/ S0143-6228(01)00019-4.]

Sherrouse, B.C., Semmens, D.J., and Clement, J.M., 2014, An application of Social Values for Ecosystem Services (SolVES) to three national forests in Colorado and Wyoming: Ecological Indicators, v. 36, p. 68-79. [Also available at https://doi.org/10.1016/j.ecolind.2013.07.008.] 


\section{Appendix 2. Preparing and Loading User-Supplied Data to the "solves" Database}

This appendix contains instructions for preparing and loading the "solves" database with all required, user-supplied data including vector and tabular survey data and environmental raster data. After completing the steps outlined in these instructions, users can proceed to use Social Values for Ecosystem Services (SolVES) to analyze and view their own data as described in the "Project Setup," "Analyze Survey Data," "Transfer Values," and "View Results" sections.

\section{Preparing Data for the "solves" Database}

Users should prepare all required data as described in the "PostgreSQL Source Database Structure and Contents" section of appendix 1. Users should also review appendix 3 and the sample data provided for the Pike and San Isabel National Forests before preparing their data. Vector data should be in shapefile format. Raster data should be in Tagged Image File Format (TIF) or American Standard Code for Information Interchange (ASCII) format, and all raster data must have identical extents. Tabular data should be in comma-separated values (CSV) format with the required field names included. Users should use Windows to navigate to the $\backslash$ SolVES $\backslash$ Data $\backslash$ User_Data folder, which is provided as a staging area for preparing user-supplied data and copy their data to the corresponding rasters, tables, and vectors folders (fig. 2.1)

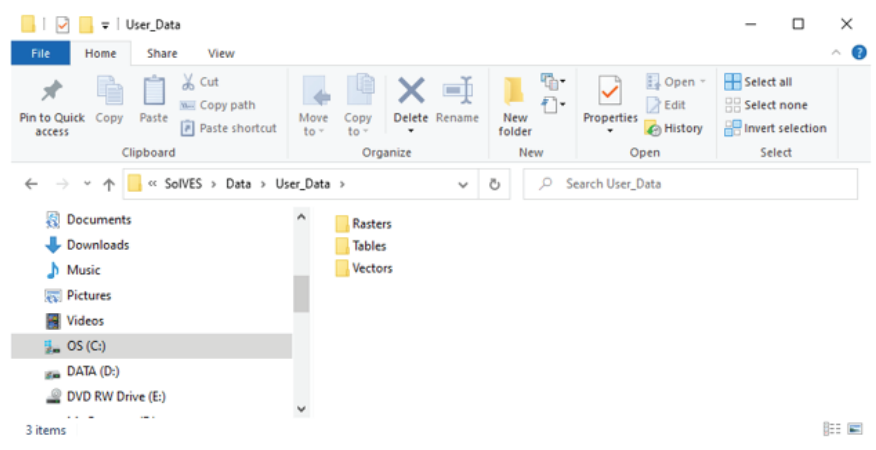

Figure 2.1. Social Values for Ecosystem Services, version 4.0, directory location for preparing user-supplied data.

\section{Loading Data to the "solves" Database}

Because SolVES was designed to work in conjunction with a PostgreSQL database, user-supplied data must be imported or loaded into the solves database (fig. 2.2). Separate tools and methods described below are currently required to load vector, tabular, and raster data. After all required vector layers, tables, and rasters have been prepared and loaded, users can proceed with using SolVES to analyze their data.

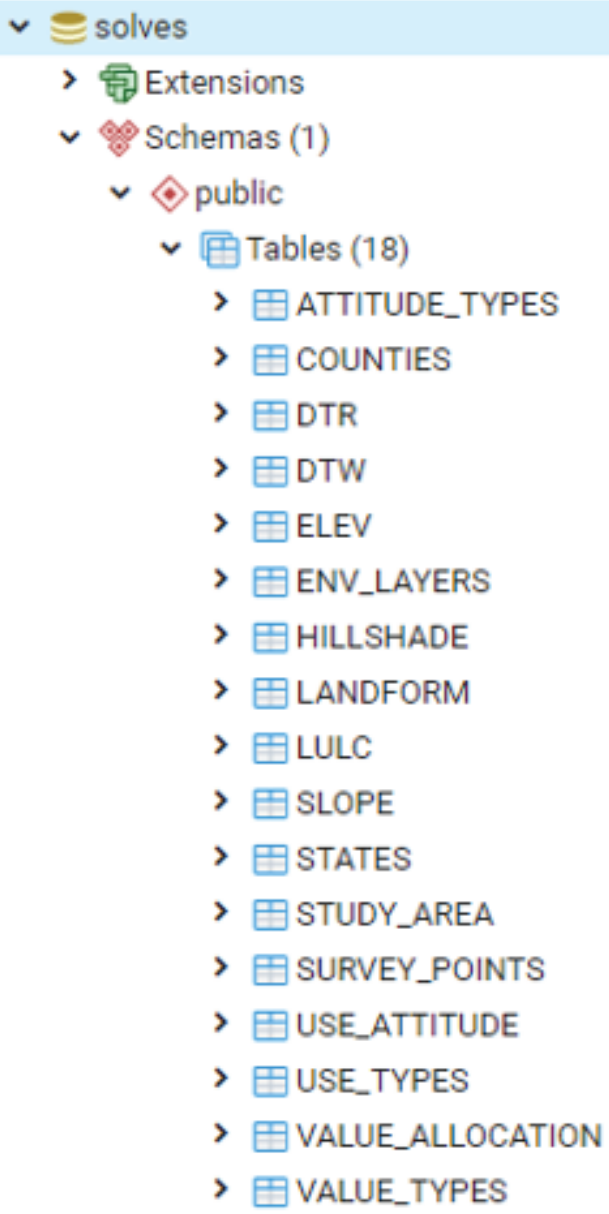

Figure 2.2. An example of the "solves" database with required data as viewed in pgAdmin 4. 


\section{Loading Vector Data}

The required vector layers, "STUDY_AREA" and "SURVEY_POINTS" can be loaded into the solves database using the PostGIS 2.0 Shapefile and DBF Loader Exporter tool. These layers should be in shapefile format before loading. Complete the following steps to load vector layers into the solves database.

1. Enter "postgis" in the Windows search tool located on the taskbar and then select the PostGIS 2.0 Shapefile and DBF Loader Exporter tool (fig. 2.3).

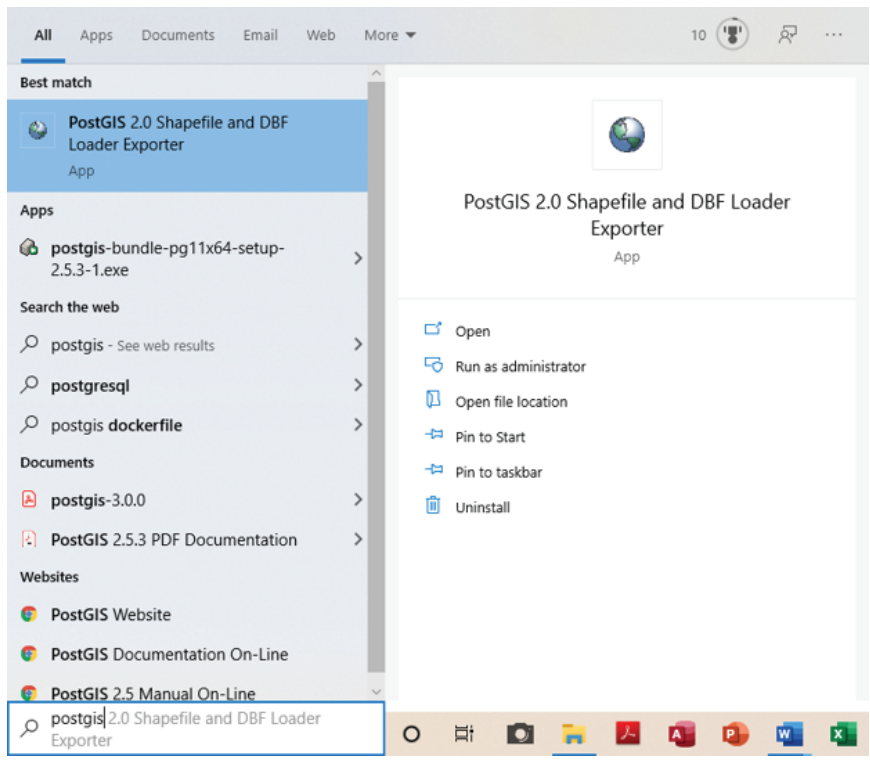

Figure 2.3. Accessing the PostGIS 2.5.3 shapefile loader in Windows.

2. Select "View connection details..." (fig. 2.4).

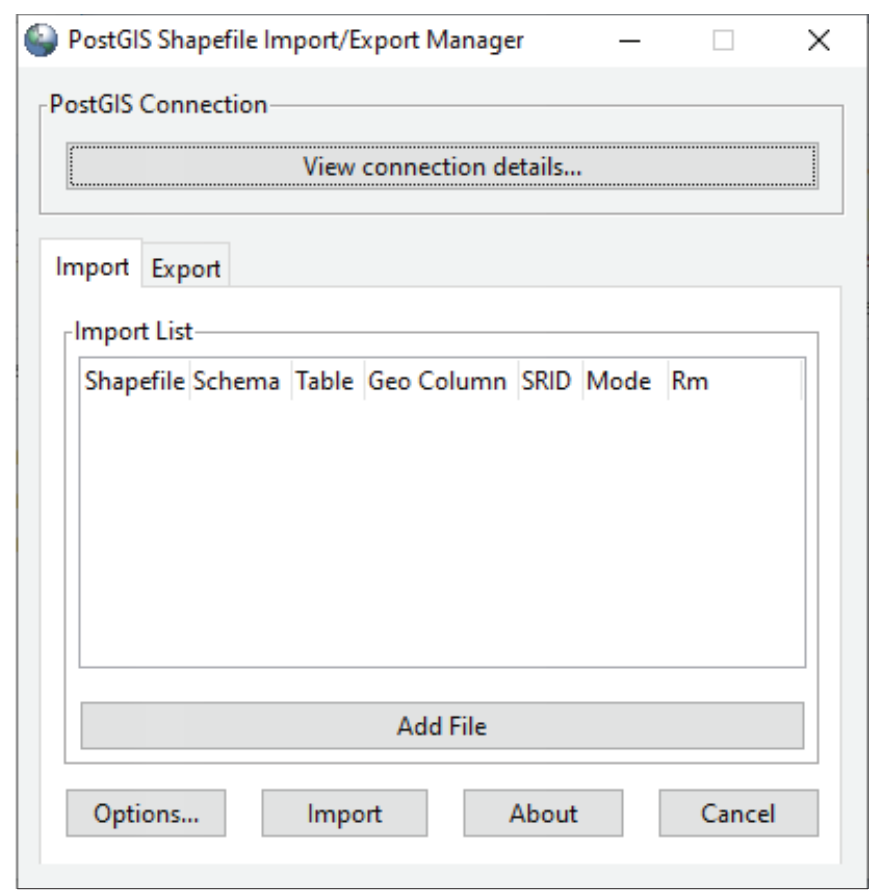

Figure 2.4. Accessing database connection details in Windows.

3. Enter "postgres" as the username and password, confirm the server host is "localhost" and the port is " 5432 ," and enter "solves" as the Database. Then select "OK" (fig. 2.5).

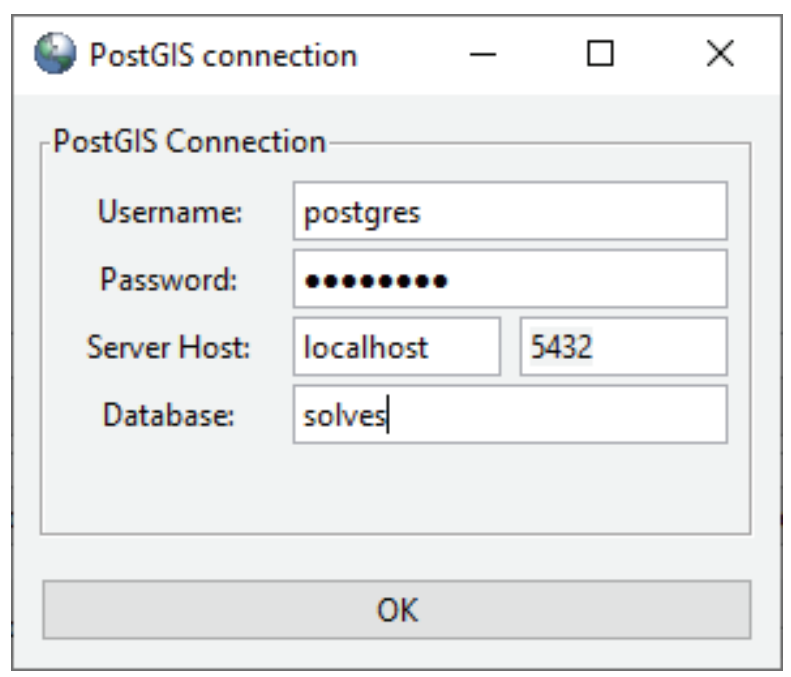

Figure 2.5. Connecting to the "solves" database in the PostGIS 2.5.3 shapefile loader. 
4. Select "Add File" and navigate to \SolVES\Data $\backslash$ User DatalVectors (fig. 2.6).

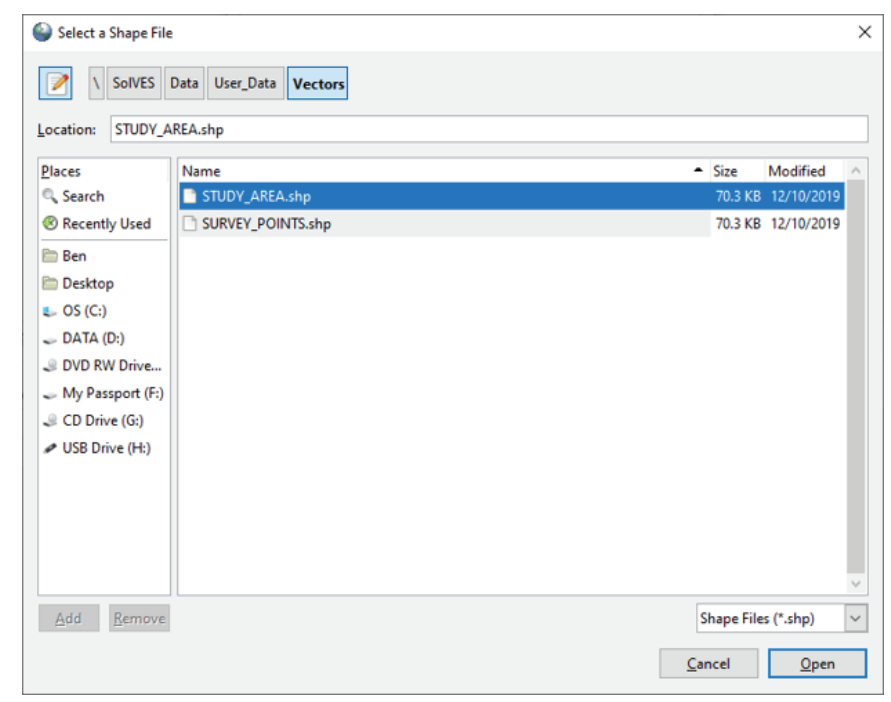

Figure 2.6. Selecting user-supplied vector data in the PostGIS 2.5 .3 shapefile loader.

5. Select all files and select "Open."

6. Select "Import" and wait for the "Shapefile import completed" message to appear in the log window (fig. 2.7).

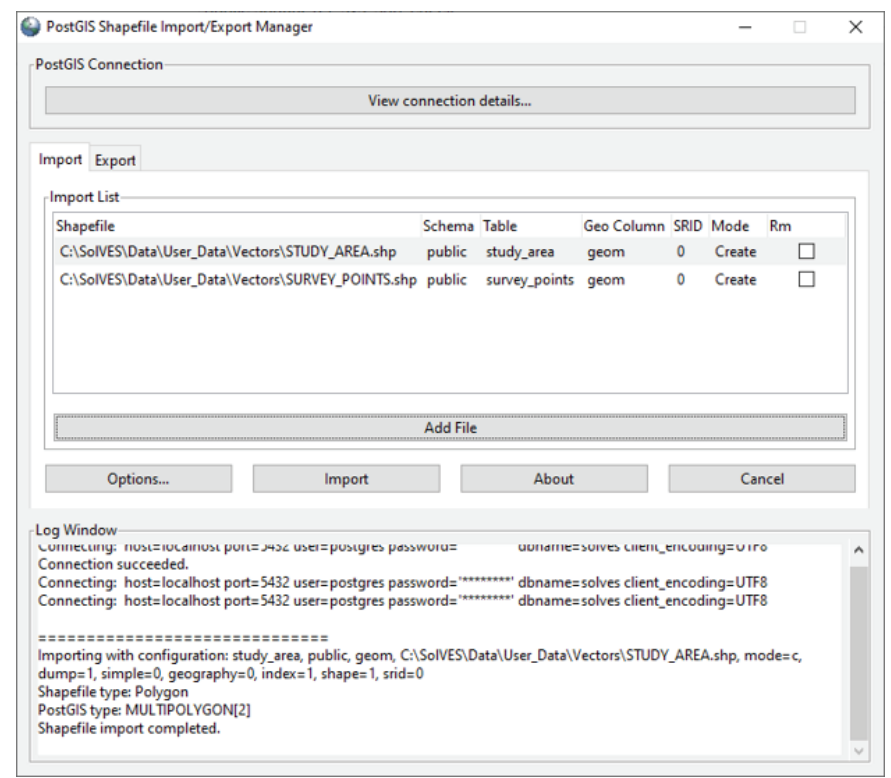

Figure 2.7. Completion of vector data import to "solves" database in the PostGIS 2.5.3 shapefile loader.
7. Close the PostGIS 2.0 Shapefile and DBF Loader Exporter tool and continue to the "Loading Table Data" section. 


\section{Loading Table Data}

The required table data can be loaded into the solves database using pgAdmin 4. These data should be in CSV format before loading. The "ATTITUDE_TYPES" table does not need to be loaded unless changes were made to it as described in the "Recoding the ATTITUDE_TYPES Table" section.

1. Right-click the table to be loaded and select "Import/ Export..." (fig. 2.8).

\section{Pg/Admin File $\vee$ Object $\vee$ Tools $\vee$ Help $\vee$}

Browser
> Eanguages

$\checkmark$ Schemas (1)

$\checkmark \diamond$ public

> ${ }_{\mathrm{B} \downarrow} \downarrow$ Collations

$>$ Domains

> FTS Configurations

$>$ I0 FTS Dictionaries

> Aa FTS Parsers

> FTS Templates

> Fi甲 Foreign Tables

> (乡) Functions

> Materialized Views

>1.3 Sequences

$\checkmark$ 宙Tables (18)

> 巴ATTITUDE_TYPES

> BCOUNTIES

> 巴DTR

> 巴DTW

> 由ELEV

> 巴ENV_LAYERS

> ĐHILLSHADE

> 巴LANDFORM

> 由LULC

> ⿴囗十 SLOPE

> 由STATES

> BSTUDY_AREA

> GSURVEY_POII

> 巴USE_ATTITUL.

> 巴USE_TYPES

> 由VAlue_allo

> 由VAlue_type

> $巴$ spatial_ref_sy

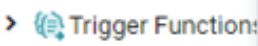

>
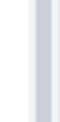

Import/Export..

Maintenance...

Scripts

Truncate

$>$

Backup...

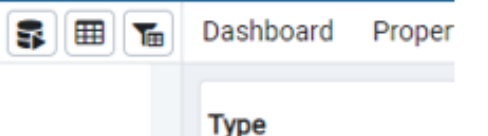

> (U) Procedures
Type
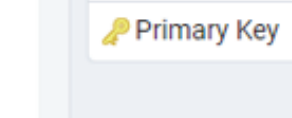

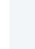

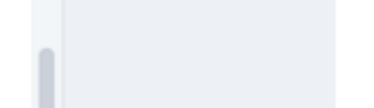

Import/Export data - table 'ENV LAYERS'

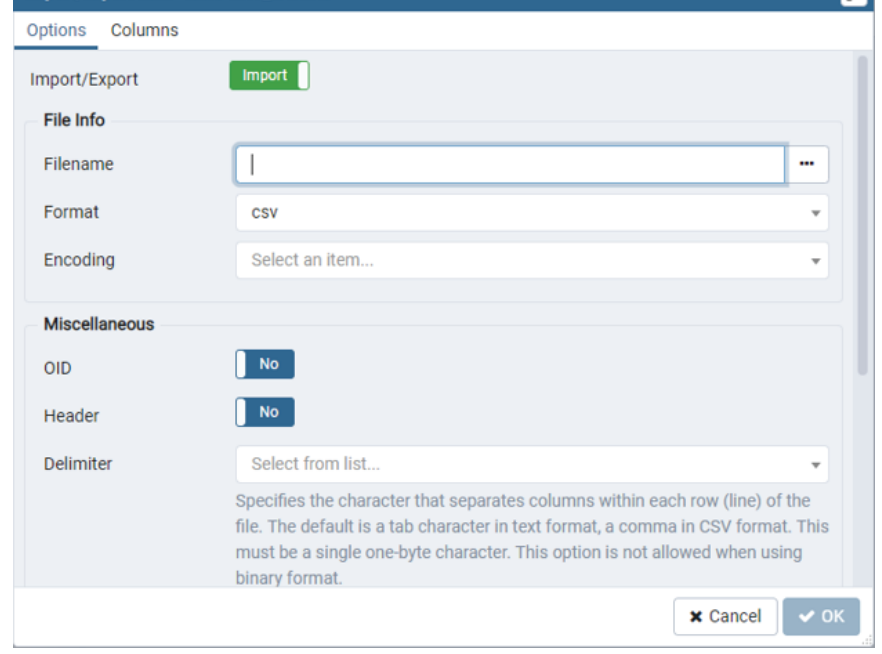

Figure 2.9. Selecting tables to import user-supplied data to the

"solves" database in pgAdmin 4.

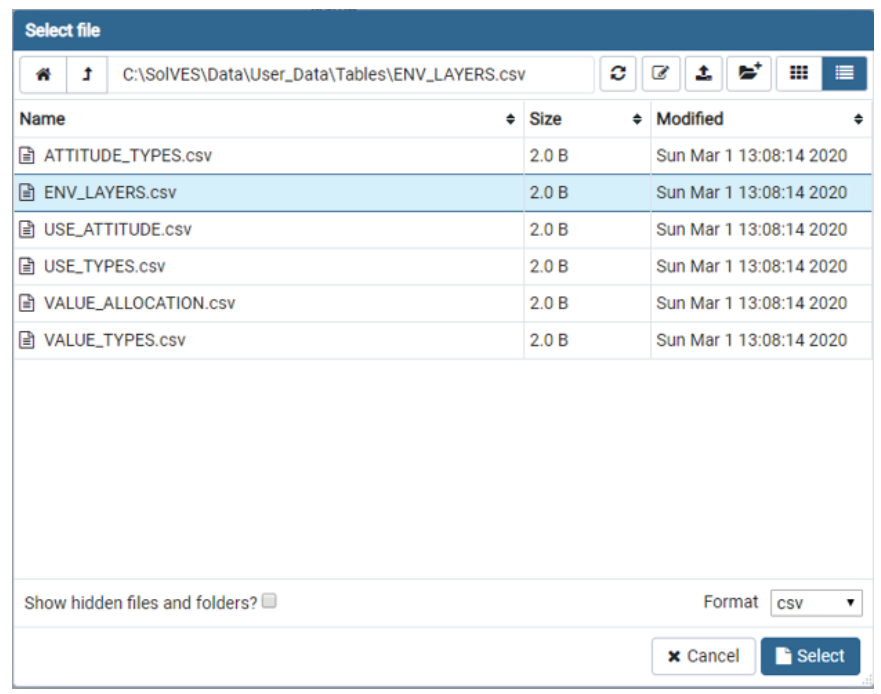

Figure 2.10. Selecting tables to import user-supplied data to the "solves" database in pgAdmin 4.

3. Highlight the table to be loaded and choose "Select."

2. Select the "Import" option on the import/export data filename field (fig. 2.10).

Figure 2.8. Selecting tables to import user-supplied data to the "solves" database in pgAdmin 4. 
4. Select the columns tab (fig. 2.11).

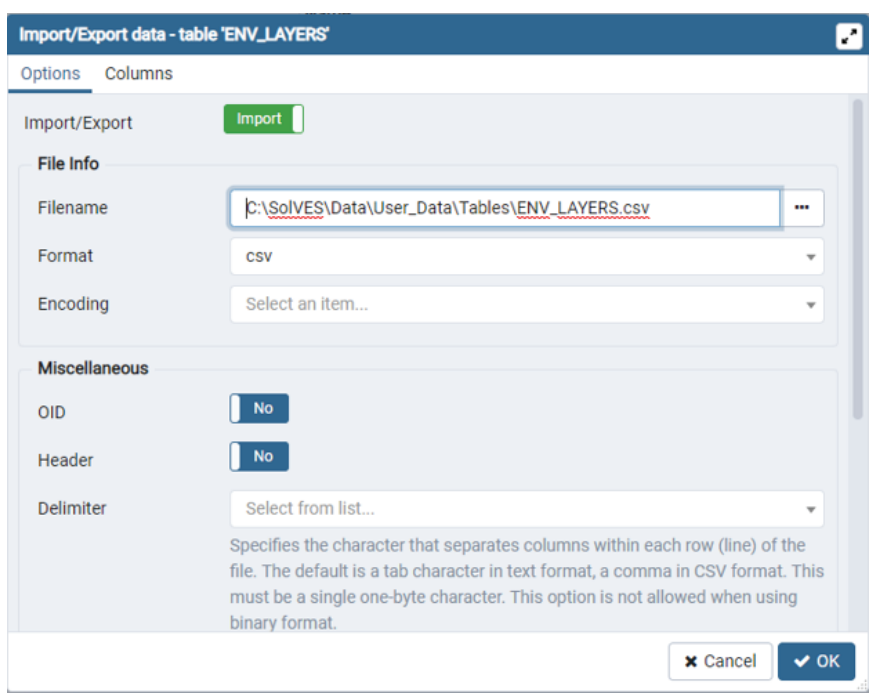

Figure 2.11. Example of a table selected for importing to the "solves" database in pgAdmin 4.

5. Select the "id" field from columns to import to exclude it from the import (fig. 2.12).

Note.-The "id" field will be automatically updated during the import process.

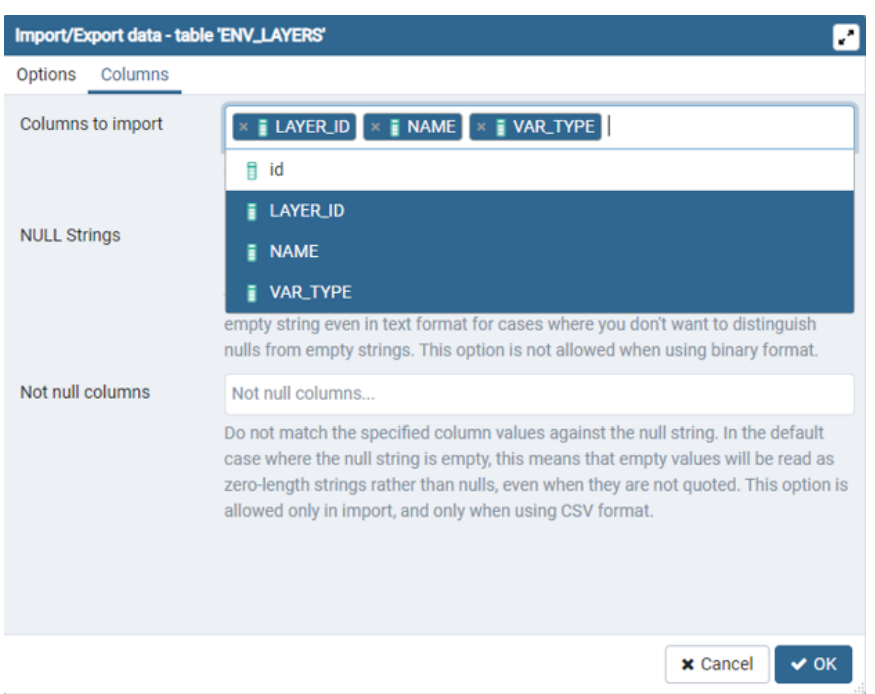

Figure 2.12. Removing the "id" field from the table import in pgAdmin 4.
6. Enter two single quotes (") into the "NULL Strings" field so that fields allowed to have null values or empty strings will be included in the import. Then select "OK" (fig. 2.13).

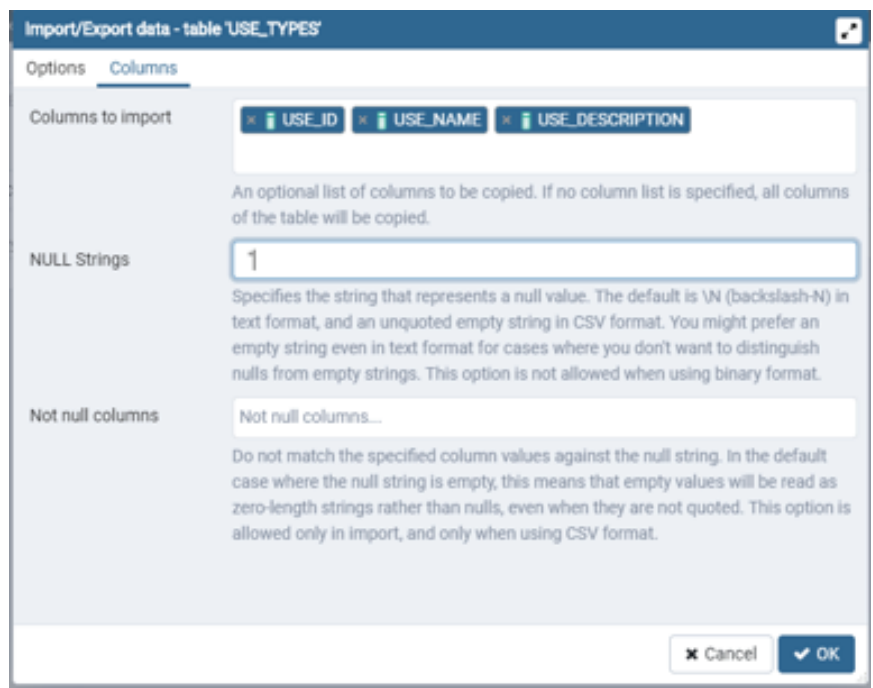

Figure 2.13. Updating the "NULL Strings" settings in pgAdmin 4.

7. Confirm a successfully completed message appears when the table has finished importing (fig. 2.14).

\section{Copying table data}

Copying table data 'public.USE_TYPES' on database 'solves' and server (localhost:5432)

Thu Feb 132020 11:25:54 GMT-0500 (Eastern Standard Time)

\begin{tabular}{|c|c|c|}
\hline (J) 0.08 seconds & (3) More details... & ® Stop Process \\
\hline
\end{tabular}

Figure 2.14. Successful import completion message in pgAdmin 4 .

8. Repeat steps $1-7$ to import all required tables. 


\section{Loading Raster Data}

Environmental rasters can be loaded into the solves database by using the Windows command prompt. These data should be in a TIF or ASCII format before loading. Users should also confirm that all rasters have identical extents before loading.

1. Enter "cmd" in the Windows task bar and select the command prompt (fig. 2.15).

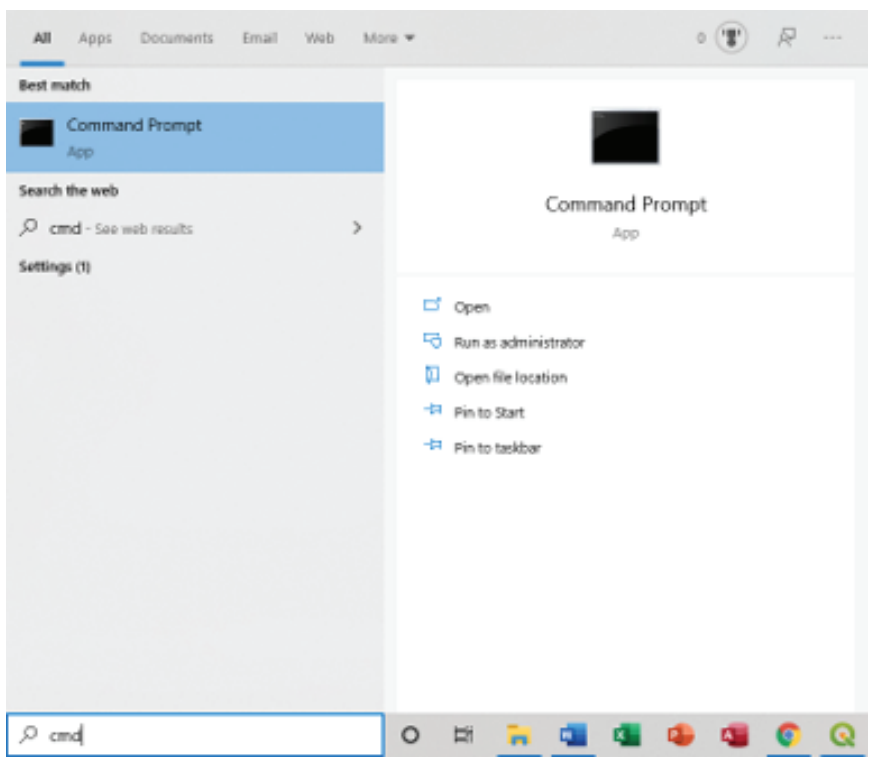

Figure 2.15. Accessing the Windows command prompt.

2. Change the directory to the PostgreSQL bin directory by typing the command "cd C: $\mid$ Program Files $\backslash$ PostgreSQL $\backslash 11 \backslash$ bin" at the command prompt (see highlighted text in fig. 2.16).

Note.-Users should substitute the directory path if it is different for their installation.

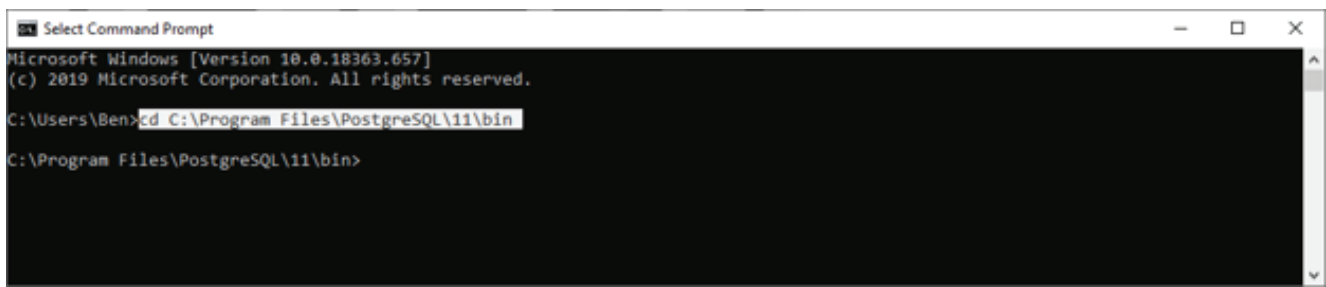

Figure 2.16. Change directory to access the PostgreSOL raster importer in Windows command prompt. 
3. Enter the command "raster2pgsql.exe -C C:|SolVES $\backslash$

DatalUser_Data|Rasters\DTR.tif | psql -d solves -U post-

gres -h localhost -p 5432" at the command prompt (see

highlighted text in fig. 2.17). but replace the path name

with the appropriate location of the SolVES directory

based on the local SolVES 4.0 installation and the name

of a user-supplied TIF or ASCII file.

4. If the postgres password is requested, type "postgres" and select "Enter."

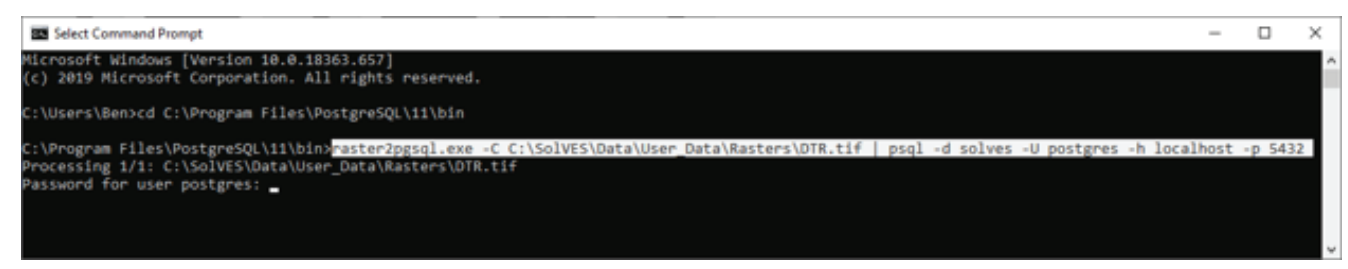

Figure 2.17. Enter raster import command and "postgres" as the password in Windows command prompt.

5. When the raster has finished loading (fig. 2.18), steps 3 and 4 can be repeated for each additional environmental raster (substituting the name of each TIF or ASCII file) until all have been loaded to the solves database.

At this point, users can proceed with completing SolVES analyses using their own data.

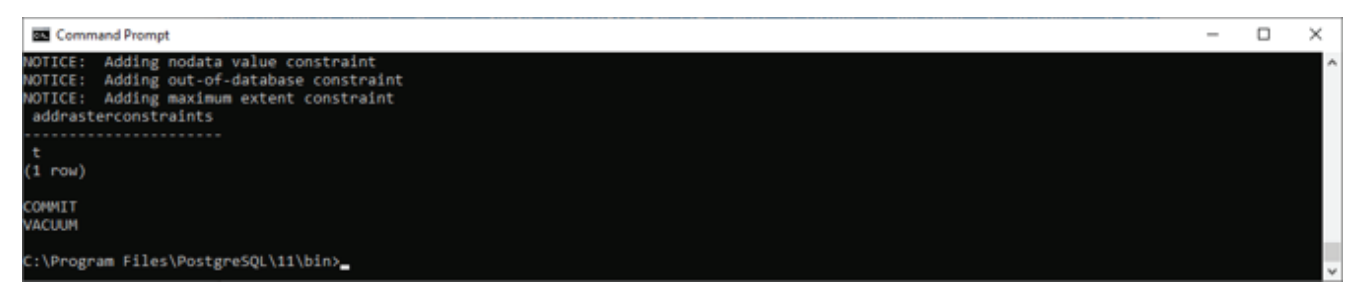

Figure 2.18. Completed raster import in Window command prompt. 


\section{Appendix 3. Social Values for Ecosystem Services, Version 4.0, Data Dictionary}

The first section of this appendix includes examples as viewed in pgAdmin 4 of required tables and vector layers in the "solves" database as described in appendix 1. These examples can also be viewed directly in pgAdmin 4 by navigating to the solves database tables section in the browser panel, right-clicking a table, and selecting View/Edit Data $>$ All Rows. The second section describes the Social Values for Ecosystem Services (SolVES), version 4.0, directory structure and contents when initially installed. The third section provides detailed descriptions of all intermediate and final data generated by SolVES during processing.

\section{Required Table and Vector Layer Examples}

The "id" fields and "geom" fields (in the case of vector data) are managed by PostgreSQL and should not be included when preparing user-provided data for loading the solves database (figs. 3.1-3.8).

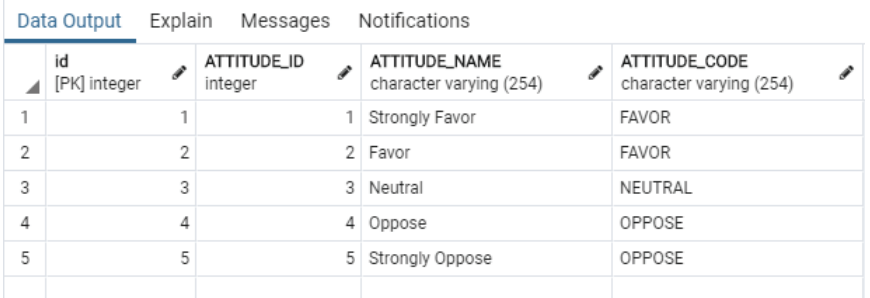

Figure 3.1. "ATTITUDE_TYPE" table in pgAdmin 4.

\begin{tabular}{|c|c|c|c|c|c|c|c|c|}
\hline \multicolumn{2}{|c|}{ Data Output } & Explain & \multicolumn{2}{|c|}{ Messages } & \multicolumn{2}{|l|}{ Notifications } & \multirow[b]{2}{*}{$\begin{array}{l}\text { VAR_TYPE } \\
\text { integer }\end{array}$} & \multirow[b]{2}{*}{ 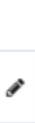 } \\
\hline$\Delta$ & $\begin{array}{l}\text { id } \\
{[\mathrm{PK}] \text { integer }}\end{array}$ & 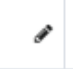 & $\begin{array}{l}\text { LAYER_ID } \\
\text { integer }\end{array}$ & 8 & $\begin{array}{l}\text { NAME } \\
\text { character varying }\end{array}$ & 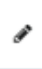 & & \\
\hline 1 & & 1 & & 1 & DTR & & & 0 \\
\hline 2 & & 2 & & 2 & DTW & & & 0 \\
\hline 3 & & 3 & & 3 & ELEV & & & 0 \\
\hline 4 & & 4 & & 4 & HILLSHADE & & & 1 \\
\hline 5 & & 5 & & 5 & LANDFORM & & & 1 \\
\hline 6 & & 6 & & 6 & LULC & & & 1 \\
\hline 7 & & 7 & & 7 & SLOPE & & & 0 \\
\hline
\end{tabular}

Figure 3.2. "ENV_LAYERS" table in pgAdmin 4.

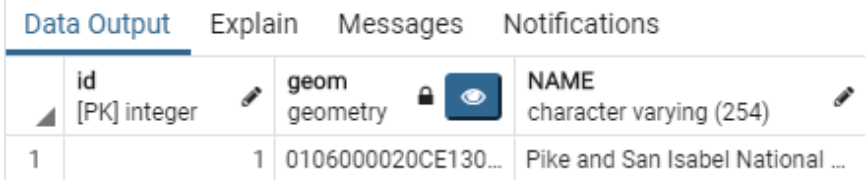

Figure 3.3. "STUDY_AREA" attribute table in pgAdmin 4.

\begin{tabular}{|c|c|c|c|c|c|c|c|c|}
\hline Data 0 & Exp & & Messages & ications & & & & \\
\hline$\Delta$ & $\begin{array}{l}\text { id } \\
\text { [PK] integer }\end{array}$ & 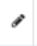 & $\begin{array}{l}\text { geom } \\
\text { geometry }\end{array}$ & $\begin{array}{l}\text { SURVEY_ID } \\
\text { integer }\end{array}$ & 子 & $\begin{array}{l}\text { VALUE_ID } \\
\text { integer }\end{array}$ & 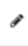 & $\begin{array}{l}\text { PLACE_NAME } \\
\text { character varying (254) }\end{array}$ \\
\hline 1 & & 1 & 0101000020CE130... & & 399 & & 1 & Maxwell Falls \\
\hline 2 & & 2 & 0101000020CE130 & & 399 & & 2 & Clear Creek Lake \\
\hline 3 & & 3 & 0101000020CE130... & & 399 & & 2 & Turquoise Lake \\
\hline 4 & & 4 & 0101000020 CE130... & & 399 & & 2 & Tarryall Reservioir \\
\hline 5 & & 5 & 0101000020CE130... & & 399 & & 5 & Through Deckers \\
\hline 6 & & 6 & 0101000020 CE $130 \ldots$ & & 399 & & 6 & St. Elmo and mining areas \\
\hline 7 & & 7 & 0101000020CE130... & & 399 & & 9 & Colorado Trail \\
\hline 8 & & 8 & 0101000020CE130... & & 399 & & 12 & Mt. Evans Wilderness Trails \\
\hline 9 & & 9 & 0101000020CE130... & & 400 & & 1 & Pretty lakes \\
\hline 10 & & 10 & 0101000020CE130... & & 400 & & 1 & Pristine lakes \\
\hline 11 & & 11 & 0101000020 CE130... & & 400 & & 2 & Nice small lakes for fishing \\
\hline 12 & & 12 & 0101000020CE130... & & 408 & & 1 & South Platte River \\
\hline 13 & & 13 & 0101000020 CE130... & & 408 & & 9 & South Platte River \\
\hline
\end{tabular}

Figure 3.4. "SURVEY_POINTS" attribute table in pgAdmin 4.

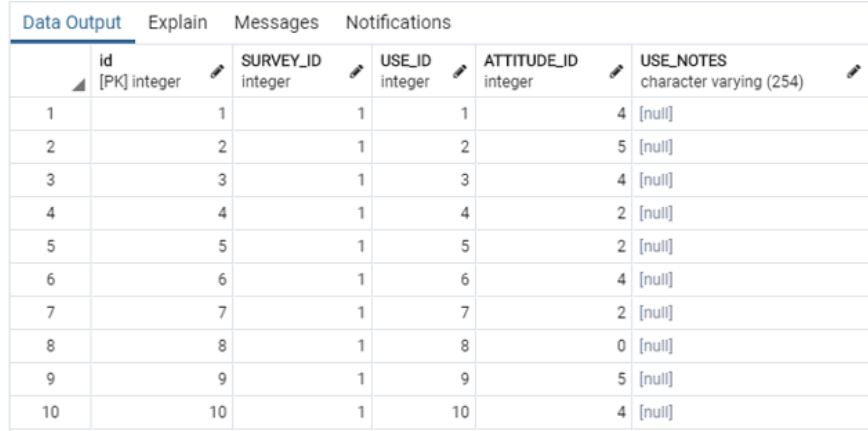

Figure 3.5. "USE_ATTITUDE" table in pgAdmin 4.

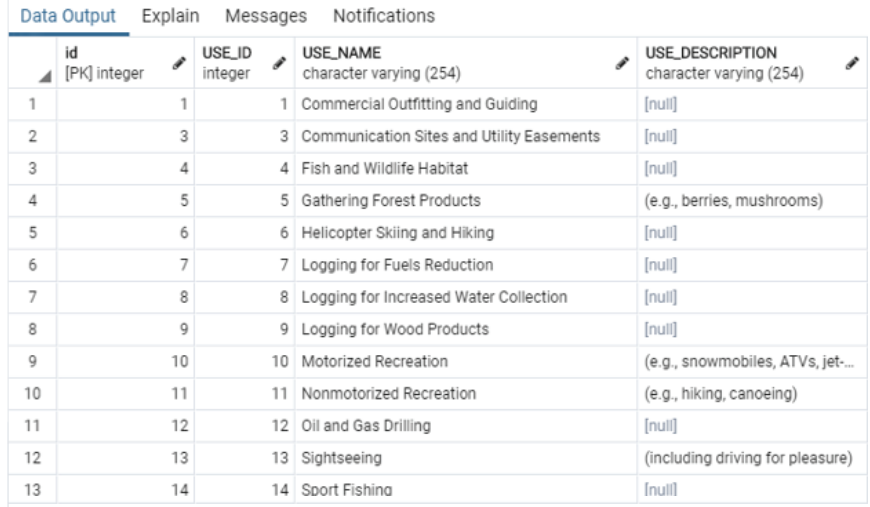

Figure 3.6. "USE_TYPES" table in pgAdmin 4. 


\begin{tabular}{|c|c|c|c|c|c|c|c|c|}
\hline \multicolumn{2}{|c|}{ Data Output } & Explain & \multirow{2}{*}{$\begin{array}{l}\text { Messages } \\
\begin{array}{l}\text { SURVEY_ID } \\
\text { integer }\end{array} \\
\end{array}$} & \multicolumn{2}{|c|}{ Notifications } & \multirow[b]{2}{*}{8} & \multirow[b]{2}{*}{$\begin{array}{l}\text { AMOUNT } \\
\text { double precision }\end{array}$} & \multirow[b]{2}{*}{ 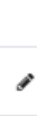 } \\
\hline$\Delta$ & $\begin{array}{l}\text { id } \\
\text { [PK] integer }\end{array}$ & $\Rightarrow$ & & $\Rightarrow$ & $\begin{array}{l}\text { VALUE_ID } \\
\text { integer }\end{array}$ & & & \\
\hline 1 & & 1 & & 1 & & 1 & & 25 \\
\hline 2 & & 2 & & 1 & & 2 & & 5 \\
\hline 3 & & 3 & & 1 & & 3 & & 5 \\
\hline 4 & & 4 & & 1 & & 4 & & 0 \\
\hline 5 & & 5 & & 1 & & 5 & & 25 \\
\hline 6 & & 6 & & 1 & & 6 & & 0 \\
\hline 7 & & 7 & & 1 & & 7 & & 5 \\
\hline 8 & & 8 & & 1 & & 8 & & 5 \\
\hline 9 & & 9 & & 1 & & 9 & & 25 \\
\hline 10 & & 10 & & 1 & & 10 & & 0 \\
\hline 11 & & 11 & & 1 & & 11 & & 0 \\
\hline 12 & & 12 & & 1 & & 12 & & 5 \\
\hline
\end{tabular}

\section{Initial Directory Structure and Contents}

The SolVES 4.0 directory structure and contents are provided in table 3.1 .

\section{Intermediate and Final Data Descriptions}

The SolVES 4.0 intermediate and final data descriptions are provided in tables 3.2 and 3.3 , respectively.

Figure 3.7. "VALUE_ALLOCATION" table in pgAdmin 4.

\begin{tabular}{|c|c|c|c|c|c|c|c|}
\hline \multicolumn{2}{|c|}{ Data Output } & Explain & \multicolumn{2}{|c|}{ Messages } & \multicolumn{3}{|l|}{ Notifications } \\
\hline$\Delta$ & $\begin{array}{l}\text { id } \\
{[\mathrm{PK}] \text { integer }}\end{array}$ & 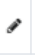 & $\begin{array}{l}\text { VALUE_ID } \\
\text { integer }\end{array}$ & 8 & $\begin{array}{l}\text { VALUE_NAME } \\
\text { character varying (255) }\end{array}$ & 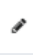 & $\begin{array}{l}\text { VALUE_DESCRIPTION } \\
\text { character varying ( } 255)\end{array}$ \\
\hline 1 & & 1 & & 1 & Aesthetic & & I enjoy the scenery, sights, so... \\
\hline 2 & & 2 & & 2 & Biodiversity & & It provides a variety of fish, wil... \\
\hline 3 & & 3 & & 3 & Cultural & & It is a place for me to continu... \\
\hline 4 & & 4 & & 4 & Economic & & It provides timber, fisheries, $\mathrm{m}$... \\
\hline 5 & & 5 & & 5 & Future & & It allows future generations to... \\
\hline 6 & & 6 & & 6 & Historic & & It has places and things of nat... \\
\hline 7 & & 7 & & 7 & Intrinsic & & I value it in and of itself, wheth.. \\
\hline 8 & & 8 & & 8 & Learning & & We can learn about the enviro... \\
\hline 9 & & 9 & & 9 & Life Sustaining & & It helps produce, preserve, cle... \\
\hline 10 & & 10 & & 10 & Recreation & & It provides a place for my favo... \\
\hline 11 & & 11 & & 11 & Spiritual & & It is a sacred, religious, or spiri... \\
\hline 12 & & 12 & & 12 & Therapeutic & & It makes me feel better, physi... \\
\hline
\end{tabular}

Figure 3.8. "VALUE_TYPES" table in pgAdmin 4. 
Table 3.1. Structure and contents of the Social Values for Ecosystem Services, version 4.0, directory.

[NA, not applicable; TIF, Tagged Image File Format; ASCII, American Standard Code for Information Interchange; CSV, comma-separated values; SolVES, Social Values for Ecosystem Services]

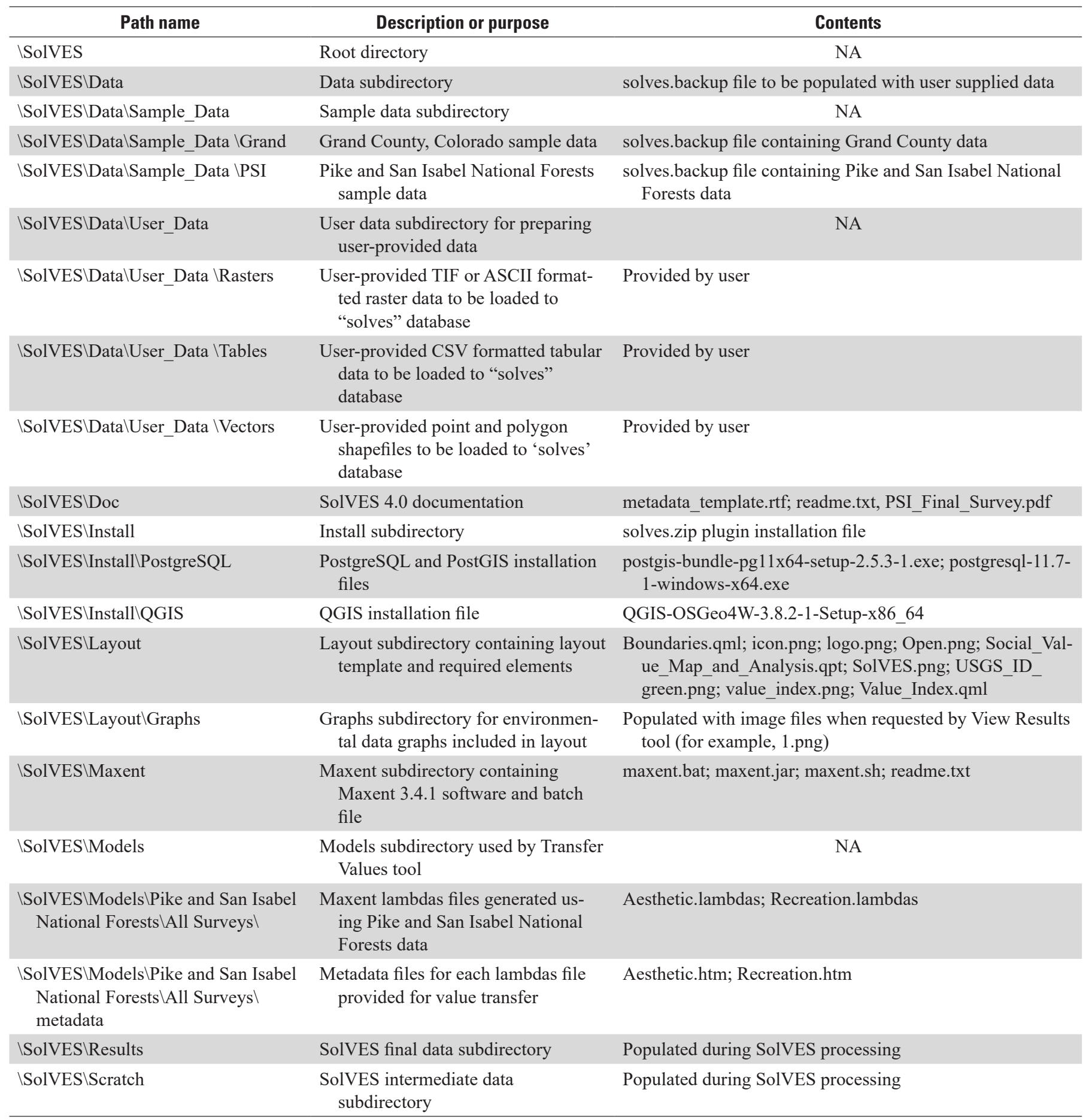


[The last three columns indicate which tools are responsible for each output, analyze survey data by survey subgroup (ASDsub), analyze survey data by social-value type (ASDval), or transfer values (TV). SolVES, Social Values for Ecosystem Services; NA, not applicable]

\begin{tabular}{|c|c|c|c|c|c|c|}
\hline Path name & Description or purpose & Contents & Content description & ASDsub & ASDval & TV \\
\hline \SolVES $\backslash$ Scratch & $\begin{array}{l}\text { SolVES intermediate data } \\
\text { subdirectory }\end{array}$ & NA & NA & $\mathrm{X}$ & $\mathrm{X}$ & $\mathrm{X}$ \\
\hline \multirow[t]{6}{*}{$\begin{array}{l}\text { \SolVES } \backslash \text { Scratch } \backslash[\text { Project } \\
\text { Name }] \backslash[\text { Social Value Type } \\
\text { or Survey Subgroup }] \backslash \\
\text { Rasters }\end{array}$} & \multirow[t]{6}{*}{$\begin{array}{l}\text { Intermediate raster data } \\
\text { generated during pro- } \\
\text { cessing }\end{array}$} & [Environmental Layer].tif & $\begin{array}{l}\text { An [Environmental Layer] (for example, DTR) from the } \\
\text { "solves" database that has been extracted and resampled } \\
\text { according to the "STUDY_AREA_FINAL" boundary, } \\
\text { output cell size, and identification as either continuous or } \\
\text { categorical }\end{array}$ & $\mathrm{X}$ & $\mathrm{X}$ & $X$ \\
\hline & & [Environmental Layer].tif & $\begin{array}{l}\text { An [Environmental Layer] (for example, DTR) from the } \\
\text { "solves" database that has been extracted and resampled } \\
\text { according to the "STUDY_AREA_FINAL" boundary, } \\
\text { output cell size, and identification as either continuous or } \\
\text { categorical }\end{array}$ & $\mathrm{X}$ & $\mathrm{X}$ & $\mathrm{X}$ \\
\hline & & $\begin{array}{l}\text { [Social Value Type or } \\
\text { Survey Subgroup]_kd.tif }\end{array}$ & $\begin{array}{l}\text { The kernel density surface of SURVEY_POINTS_SE- } \\
\text { LECT associated with each [Social Value Type or } \\
\text { Survey Subgroup] (for example, Aesthetic or Motorized } \\
\text { Recreation OPPPOSE0 included in the supplied survey } \\
\text { data and weighted or not, based on user input, by the } \\
\text { sum of associated AMOUNT field values selected from } \\
\text { the VALUE_ALLOCATION table.) }\end{array}$ & $\mathrm{X}$ & $\mathrm{X}$ & NA \\
\hline & & $\begin{array}{l}\text { [Social Value Type or } \\
\quad \text { Survey Subgroup]_10.tif }\end{array}$ & $\begin{array}{l}\text { The result of Maxent logistic output [Social Value Type or } \\
\text { Survey Subgroup].asc multiplied by the Maximum Value } \\
\text { Index calculated for each social value type or survey } \\
\text { subgroup selected by the user. }\end{array}$ & $\mathrm{X}$ & $\mathrm{X}$ & $\mathrm{X}$ \\
\hline & & $\begin{array}{l}\text { [Social Value Type or } \\
\text { Survey Subgroup]_x.tif }\end{array}$ & $\begin{array}{l}\text { The result of Maxent logistic output [Social Value Type of } \\
\text { Survey Subgroup].asc multiplied by the Maximum Value } \\
\text { Index calculated for each social value type selected by } \\
\text { the user and again multiplied by } 10 \text { to rescale the data to } \\
\text { a } 100 \text {-point scale. }\end{array}$ & $\mathrm{X}$ & $\mathrm{X}$ & $\mathrm{X}$ \\
\hline & & $\begin{array}{l}\text { [Social Value Type or } \\
\quad \text { Survey Subgroup]100.tif }\end{array}$ & $\begin{array}{l}\text { The result of the [Social Value Type or Survey } \\
\text { Subgroup]_x.tif being converted to an integer data layer, } \\
\text { which is used to calculate zonal statistics for all environ- } \\
\text { mental layers included in a model. }\end{array}$ & $\mathrm{X}$ & $\mathrm{X}$ & $\mathrm{X}$ \\
\hline \multirow[t]{2}{*}{$\begin{array}{l}\text { SolVES } \backslash \text { Scratch } \backslash[\text { Project } \\
\text { Name }] \backslash[\text { Social Value Type } \\
\text { or Survey Subgroup }] \backslash \\
\text { Tables }\end{array}$} & & threshold.txt & $\begin{array}{l}\text { A file containing the flag "threshold" if the user selects yes } \\
\text { to include threshold features and empty string if the user } \\
\text { selects no to include threshold features. The flag is ap- } \\
\text { pended to the maxent.bat file for processing. }\end{array}$ & $\mathrm{X}$ & $\mathrm{X}$ & NA \\
\hline & & alloc_sel.dbf & $\begin{array}{l}\text { Table containing results of "solves" database table joins or } \\
\text { value allocation sums }\end{array}$ & NA & $\mathrm{X}$ & NA \\
\hline
\end{tabular}


Table 3.2. Social Values for Ecosystem Services, version 4.0, intermediate data.-Continued

[The last three columns indicate which tools are responsible for each output, analyze survey data by survey subgroup (ASDsub), analyze survey data by social-value type (ASDval), or transfer values (TV). SolVES, Social Values for Ecosystem Services; NA, not applicable]

\begin{tabular}{|c|c|c|c|c|c|c|}
\hline Path name & Description or purpose & Contents & Content description & ASDsub & ASDval & TV \\
\hline & & alloc_sub.dbf & $\begin{array}{l}\text { Table containing results of "solves" database table joins of } \\
\text { value allocation sums }\end{array}$ & NA & $\mathrm{X}$ & NA \\
\hline & & alloc_val.dbf & $\begin{array}{l}\text { Table containing results of "solves" database table joins or } \\
\text { value allocation sums }\end{array}$ & $\mathrm{X}$ & $\mathrm{X}$ & NA \\
\hline & & useatt_ut.dbf & $\begin{array}{l}\text { Table containing results of "solves" database table joins or } \\
\text { value allocation sums }\end{array}$ & $\mathrm{X}$ & $\mathrm{X}$ & NA \\
\hline & & useatt_sel.dbf & $\begin{array}{l}\text { Table containing results of "solves" database table joins or } \\
\text { value allocation sums }\end{array}$ & $\mathrm{X}$ & $\mathrm{X}$ & NA \\
\hline & & useatt_utat.dbf & $\begin{array}{l}\text { Table containing results of "solves" database table joins or } \\
\text { value allocation sums }\end{array}$ & $\mathrm{X}$ & $\mathrm{X}$ & NA \\
\hline & & sub_fld.dbf & $\begin{array}{l}\text { Table containing results of "solves" database table joins or } \\
\text { value allocation sums }\end{array}$ & $\mathrm{X}$ & $\mathrm{X}$ & NA \\
\hline & & sub_sel.dbf & $\begin{array}{l}\text { Table containing results of "solves" database table joins or } \\
\text { value allocation sums }\end{array}$ & $\mathrm{X}$ & NA & NA \\
\hline & & sub_val.dbf & $\begin{array}{l}\text { Table containing results of "solves" database table joins or } \\
\text { value allocation sums }\end{array}$ & $\mathrm{X}$ & NA & NA \\
\hline & & val_tot.dbf & $\begin{array}{l}\text { Table containing results of "solves" database table joins or } \\
\text { value allocation sums }\end{array}$ & $\mathrm{X}$ & $\mathrm{X}$ & NA \\
\hline & & toggle_type.txt & $\begin{array}{l}\text { A file containing flags to identify categorical environmental } \\
\text { variables. These flags are appended to the maxent.bat file } \\
\text { for processing. }\end{array}$ & $\mathrm{X}$ & $\mathrm{X}$ & NA \\
\hline & & $\begin{array}{l}\text { [Social Value Type or } \\
\text { Survey Subgroup] } \\
\text { avgnnhtml.txt }\end{array}$ & $\begin{array}{l}\text { Files containing the html output of the average nearest } \\
\text { neighbor tool used for populating the list of social value } \\
\text { types or survey subgroups available for selection using } \\
\text { the Analyze Survey Data tool }\end{array}$ & $\mathrm{X}$ & $\mathrm{X}$ & NA \\
\hline & & AVG_NNx.csv & $\begin{array}{l}\text { Used when extracting average nearest neighbor statistics } \\
\text { from html output }\end{array}$ & $\mathrm{X}$ & $\mathrm{X}$ & NA \\
\hline & & AVG_NNy.csv & $\begin{array}{l}\text { Used when extracting average nearest neighbor statistics } \\
\text { from html output }\end{array}$ & $\mathrm{X}$ & $\mathrm{X}$ & NA \\
\hline & & avgnn.txt & $\begin{array}{l}\text { Used when extracting average nearest neighbor statistics } \\
\text { from html output }\end{array}$ & $\mathrm{X}$ & $\mathrm{X}$ & NA \\
\hline & & avgnn2.txt & $\begin{array}{l}\text { Used when extracting average nearest neighbor statistics } \\
\text { from html output }\end{array}$ & $\mathrm{X}$ & $\mathrm{X}$ & NA \\
\hline
\end{tabular}


[The last three columns indicate which tools are responsible for each output, analyze survey data by survey subgroup (ASDsub), analyze survey data by social-value type (ASDval), or transfer values (TV). SolVES, Social Values for Ecosystem Services; NA, not applicable]

\begin{tabular}{|c|c|c|c|c|c|c|}
\hline Path name & Description or purpose & Contents & Content description & ASDsub & ASDval & TV \\
\hline & & toggle_spec.txt & $\begin{array}{l}\text { A file containing flags to identify all social value types or } \\
\text { survey subgroups not selected by the user for analy- } \\
\text { sis. These flags are appended to the maxent.bat file for } \\
\text { processing. }\end{array}$ & $\mathrm{X}$ & $\mathrm{X}$ & NA \\
\hline \multirow{8}{*}{$\begin{array}{l}\text { ISolVES } \backslash \text { Scratch } \backslash[\text { Project } \\
\text { Name }] \backslash[\text { Social Value Type } \\
\text { or Survey Subgroup }] \backslash \\
\text { Vectors }\end{array}$} & & $\begin{array}{l}\text { [Social Value Type or } \\
\text { Survey Subgroup]_select. } \\
\text { shp }\end{array}$ & $\begin{array}{l}\text { Shapefile containing survey points selected for the specified } \\
\text { social value type or survey subgroup }\end{array}$ & $\mathrm{X}$ & $\mathrm{X}$ & NA \\
\hline & & alloc_pts.shp & $\begin{array}{l}\text { Shapefile containing results of survey points joined with } \\
\text { other tables in the "solves" database or allocation sums. }\end{array}$ & & $X$ & NA \\
\hline & & pts_join.shp & $\begin{array}{l}\text { Shapefile containing results of survey points joined with } \\
\text { other tables in the "solves" database or allocation sums. }\end{array}$ & $\mathrm{X}$ & $\mathrm{X}$ & NA \\
\hline & & pts_select.shp & $\begin{array}{l}\text { Shapefile containing results of survey points joined with } \\
\text { other tables in the "solves" database or allocation sums. }\end{array}$ & $\mathrm{X}$ & $\mathrm{X}$ & NA \\
\hline & & pts_sum.shp & $\begin{array}{l}\text { Shapefile containing results of survey points joined with } \\
\text { other tables in the "solves" database or allocation sums. }\end{array}$ & $\mathrm{X}$ & $X$ & NA \\
\hline & & sub_pts.shp & $\begin{array}{l}\text { Shapefile containing results of survey points joined with } \\
\text { other tables in the "solves" database or allocation sums. }\end{array}$ & $\mathrm{X}$ & NA & NA \\
\hline & & val_pts.shp & $\begin{array}{l}\text { Shapefile containing results of survey points joined with } \\
\text { other tables in the "solves" database or allocation sums. }\end{array}$ & $\mathrm{X}$ & $\mathrm{X}$ & NA \\
\hline & & zone_vi.shp & $\begin{array}{l}\text { Shapefile used for calculating zonal statistics from environ- } \\
\text { mental data. }\end{array}$ & NA & NA & $\mathrm{X}$ \\
\hline \multirow{4}{*}{$\begin{array}{l}\text { \SolVES } \backslash \text { Scratch } \backslash[\text { Project } \\
\text { Name }] \backslash[\text { Social Value Type } \\
\text { or Survey Subgroup }] \backslash \\
\text { Vectors } \backslash[\text { Social Value Type } \\
\text { or Survey Subgroup] }\end{array}$} & & zone_dis.shp & $\begin{array}{l}\text { Shapefile used in the processing of generating a zonal layer } \\
\text { for calculating statistics from environmental data for the } \\
\text { specified social value type or survey subgroup. }\end{array}$ & $X$ & $X$ & $\mathrm{X}$ \\
\hline & & zone_fix.shp & $\begin{array}{l}\text { Shapefile used in the processing of generating a zonal layer } \\
\text { for calculating statistics from environmental data for the } \\
\text { specified social value type or survey subgroup. }\end{array}$ & $\mathrm{X}$ & $\mathrm{X}$ & $\mathrm{X}$ \\
\hline & & zone_poly.shp & $\begin{array}{l}\text { Shapefile used in the processing of generating a zonal layer } \\
\text { for calculating statistics from environmental data for the } \\
\text { specified social value type or survey subgroup. }\end{array}$ & $\mathrm{X}$ & $\mathrm{X}$ & $\mathrm{X}$ \\
\hline & & zonevi_tmp.shp & $\begin{array}{l}\text { Shapefile used in the processing of generating a zonal layer } \\
\text { for calculating statistics from environmental data for the } \\
\text { specified social value type or survey subgroup. }\end{array}$ & $\mathrm{X}$ & $\mathrm{X}$ & $\mathrm{X}$ \\
\hline
\end{tabular}


Table 3.3. Social Values for Ecosystem Services, version 4.0, final data.

The last three columns indicate which tools are responsible for each output, analyze survey data by survey subgroup (ASDsub), analyze survey data by social-value type (ASDval), or transfer values (TV). SolVES, Social Values for Ecosystem Services; NA, not applicable; ASCII, American Standard Code for Information Interchange; AUC, Area Under the Curve]

\begin{tabular}{|c|c|c|c|c|c|c|}
\hline Path name & Description or purpose & Contents & Content description & ASDsub & ASDval & TV \\
\hline \SolVES\Results & SolVES final data subdirectory & NA & NA & $\mathrm{X}$ & $\mathrm{X}$ & $\mathrm{X}$ \\
\hline \multirow[t]{2}{*}{ ISolVES \Results $\backslash[$ Project Name] } & \multirow[t]{2}{*}{$\begin{array}{l}\text { Project-level directory based on } \\
\text { user-entered [Project Name] }\end{array}$} & cat_var.txt & $\begin{array}{l}\text { A file containing the name of all environmental } \\
\text { layers identified as categorical }\end{array}$ & $\mathrm{X}$ & $\mathrm{X}$ & $\mathrm{X}$ \\
\hline & & env_var.txt & $\begin{array}{l}\text { A file containing the name of all environmental } \\
\text { layers }\end{array}$ & $\mathrm{X}$ & $\mathrm{X}$ & $\mathrm{X}$ \\
\hline $\begin{array}{l}\backslash \text { SolVES } \backslash \text { Results } \backslash[\text { Project Name }] \backslash \\
\text { [Social Value Type or Survey } \\
\text { Subgroup] }\end{array}$ & $\begin{array}{l}\text { Final data generated during } \\
\text { processing. }\end{array}$ & NA & NA & NA & NA & NA \\
\hline 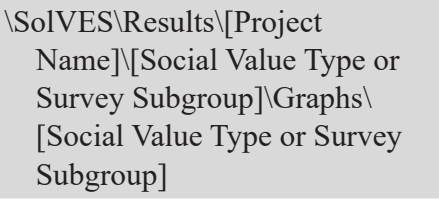 & $\begin{array}{l}\text { Environmental data graphs gener- } \\
\text { ated for the selected social } \\
\text { value type or survey subgroup }\end{array}$ & {$[\#]$.png } & $\begin{array}{l}\text { Image files numbered in the order they are created } \\
\text { for each environmental data layer and corre- } \\
\text { sponding to a position in the final map layout. }\end{array}$ & $\mathrm{X}$ & $\mathrm{X}$ & $\mathrm{X}$ \\
\hline \multirow{3}{*}{$\begin{array}{l}\backslash \text { SolVES } \backslash \text { Results } \backslash[\text { Project Name }] \backslash \\
\text { [Social Value Type or Survey } \\
\text { Subgroup }] \backslash \text { Log }\end{array}$} & \multirow[t]{3}{*}{$\begin{array}{l}\text { Location of SolVES and Maxent } \\
\text { processing information }\end{array}$} & asd.txt & $\begin{array}{l}\text { Log file generated when the Analyze Survey Data } \\
\text { tool is run. }\end{array}$ & $\mathrm{X}$ & $\mathrm{X}$ & NA \\
\hline & & tv.txt & $\begin{array}{l}\text { Log file generated when the Transfer Values tool } \\
\text { is run. }\end{array}$ & & & $\mathrm{X}$ \\
\hline & & maxent_bat.txt & $\begin{array}{l}\text { Copy of the maxent.bat file contents generated for } \\
\text { each SolVES analysis. }\end{array}$ & $X$ & $\mathrm{X}$ & $X$ \\
\hline \begin{tabular}{l} 
ISolVES $\backslash$ Results $\backslash[$ Project Name]\} $\\
{\text { [Social Value Type or Survey }} \\
{\text { Subgroup]\Maxent_Data }} \\
{\text { Environmental }}\end{array}$ & $\begin{array}{l}\text { Location of environmental data } \\
\text { layers generated for Maxent } \\
\text { analysis }\end{array}$ & [Environmental Layer].asc & $\begin{array}{l}\text { An [Environmental Layer] (for example, DTR) } \\
\text { from the "solves" database that have been con- } \\
\text { verted to ASCII format for Maxent processing. }\end{array}$ & $\mathrm{X}$ & $\mathrm{X}$ & $\mathrm{X}$ \\
\hline
\end{tabular}
\end{tabular}

Environmental 
The last three columns indicate which tools are responsible for each output, analyze survey data by survey subgroup (ASDsub), analyze survey data by social-value type (ASDval), or transfer values (TV). SolVES, Social Values for Ecosystem Services; NA, not applicable; ASCII, American Standard Code for Information Interchange; AUC, Area Under the Curve]

\begin{tabular}{|c|c|c|c|c|c|c|}
\hline Path name & Description or purpose & Contents & Content description & ASDsub & ASDval & TV \\
\hline \multirow{6}{*}{$\begin{array}{l}\backslash \text { SolVES } \backslash \text { Results } \backslash[\text { Project Name }] \backslash \\
\text { [Social Value Type or Survey } \\
\text { Subgroup }] \backslash \text { Maxent_Data } \backslash \\
\text { Output }\end{array}$} & \multirow[t]{6}{*}{ Output generated by Maxent } & $\begin{array}{l}\text { [Social Value Type or } \\
\text { Survey Subgroup].asc }\end{array}$ & $\begin{array}{l}\text { Logistic output generated by SolVES in ASCII } \\
\text { format. }\end{array}$ & $\mathrm{X}$ & $\mathrm{X}$ & $\mathrm{X}$ \\
\hline & & $\begin{array}{l}\text { [Social Value Type or } \\
\text { Survey Subgroup].htm }\end{array}$ & $\begin{array}{l}\text { A summary of all output generated by Maxent } \\
\text { for the specified social value type or survey } \\
\text { subgroup. }\end{array}$ & $\mathrm{X}$ & $\mathrm{X}$ & NA \\
\hline & & $\begin{array}{l}\text { [Social Value Type or } \\
\text { Survey Subgroup]. } \\
\text { lambdas }\end{array}$ & $\begin{array}{l}\text { The model generated by Maxent describing } \\
\text { the relationship between survey points and } \\
\text { environmental variables for the specified social } \\
\text { value type or survey subgroup. }\end{array}$ & $\mathrm{X}$ & $\mathrm{X}$ & NA \\
\hline & & maxent.log & A log file of all Maxent processing. & $\mathrm{X}$ & $\mathrm{X}$ & NA \\
\hline & & maxentResults.csv & $\begin{array}{l}\text { A file containing a record of the numeric results } \\
\text { of each Maxent model including AUC statistics } \\
\text { and variable contributions. }\end{array}$ & $\mathrm{X}$ & $\mathrm{X}$ & NA \\
\hline & & $\begin{array}{l}\text { [Social Value Type or } \\
\text { Survey Subgroup]_ } \\
\text { clamping.asc }\end{array}$ & $\begin{array}{l}\text { Maxent output indicating locations where envi- } \\
\text { ronmental variable values exceeded the range } \\
\text { of a transferred model. }\end{array}$ & NA & NA & $\mathrm{X}$ \\
\hline $\begin{array}{l}\text { ISolVES\Results } \backslash[\text { Project Name }] \backslash \\
\text { [Social Value Type or Survey } \\
\text { Subgroup]\Maxent_Data } \\
\text { Samples }\end{array}$ & $\begin{array}{l}\text { Survey points generated for } \\
\text { Maxent analysis }\end{array}$ & SURVEY_POINTS.csV & $\begin{array}{l}\text { File containing the coordinates and associated so- } \\
\text { cial value type or survey subgroup of selected } \\
\text { survey points converted to ASCII format for } \\
\text { Maxent analysis. }\end{array}$ & $\mathrm{X}$ & $\mathrm{X}$ & NA \\
\hline $\begin{array}{l}\backslash \text { SolVES } \backslash \text { Results } \backslash[\text { Project Name }] \backslash \\
\text { [Social Value Type or Survey } \\
\text { Subgroup }] \backslash \text { Rasters }\end{array}$ & Location of final raster maps & $\begin{array}{l}\text { [Social Value Type or } \\
\text { Survey Subgroup].tif }\end{array}$ & $\begin{array}{l}\text { Final value-index maps generated for the speci- } \\
\text { fied social value type or survey subgroup and } \\
\text { used for generating layouts by the View Results } \\
\text { tool. }\end{array}$ & $\mathrm{X}$ & $\mathrm{X}$ & $\mathrm{X}$ \\
\hline \multirow[t]{2}{*}{$\begin{array}{l}\backslash \text { SolVES } \backslash \text { Results } \backslash[\text { Project Name }] \backslash \\
\text { [Social Value Type or Survey } \\
\text { Subgroup }] \backslash \text { Tables }\end{array}$} & \multirow[t]{2}{*}{$\begin{array}{l}\text { Location of average nearest } \\
\text { neighbor and maximum value } \\
\text { index results }\end{array}$} & AVG_NN.csv & $\begin{array}{l}\text { Summary of the number of points, } r \text {-ratios, and } \\
z \text {-scores for all social value types or survey } \\
\text { subgroups. }\end{array}$ & $\mathrm{X}$ & $\mathrm{X}$ & NA \\
\hline & & MAX_VI.csv & $\begin{array}{l}\text { Summary of the maximum value attained on the } \\
\text { value index for each social value type or survey } \\
\text { subgroup. }\end{array}$ & $\mathrm{X}$ & $\mathrm{X}$ & NA \\
\hline \multirow{3}{*}{$\begin{array}{l}\backslash \text { SolVES } \backslash \text { Results } \backslash[\text { Project Name }] \backslash \\
\text { [Social Value Type or Survey } \\
\text { Subgroup }] \backslash \text { Vectors }\end{array}$} & \multirow[t]{3}{*}{$\begin{array}{l}\text { Location of final vectors gener- } \\
\text { ated during processing }\end{array}$} & $\begin{array}{l}\text { STUDY_AREA_FINAL. } \\
\text { shp }\end{array}$ & $\begin{array}{l}\text { The final study area boundary after any buffers } \\
\text { have been applied. }\end{array}$ & $X$ & $X$ & NA \\
\hline & & $\begin{array}{l}\text { SURVEY_POINTS_SE- } \\
\text { LECT.shp }\end{array}$ & $\begin{array}{l}\text { The final set of survey points selected based on } \\
\text { their location within the final study area bound- } \\
\text { ary. }\end{array}$ & $X$ & $X$ & NA \\
\hline & & $\begin{array}{l}\text { [Environmental Layer }]_{-} \\
\text {stats.shp }\end{array}$ & $\begin{array}{l}\text { Shapefile containing zonal statistics calculated for } \\
\text { each environmental layer. }\end{array}$ & NA & NA & $X$ \\
\hline
\end{tabular}
each environmental layer. 
Table 3.3. Social Values for Ecosystem Services, version 4.0, final data.-Continued

The last three columns indicate which tools are responsible for each output, analyze survey data by survey subgroup (ASDsub), analyze survey data by social-value type (ASDval), or transfer values (TV). SolVES, Social Values for Ecosystem Services; NA, not applicable; ASCII, American Standard Code for Information Interchange; AUC, Area Under the Curve]

\begin{tabular}{|c|c|c|c|c|c|c|}
\hline Path name & Description or purpose & Contents & Content description & ASDsub & ASDval & TV \\
\hline $\begin{array}{l}\text { ISolVES } \backslash \text { Results } \backslash[\text { Project } \\
\text { Name]\[Social Value Type or } \\
\text { Survey Subgroup] } \backslash \text { Vectors } \backslash \\
\text { [Social Value Type or Survey } \\
\text { Subgroup] }\end{array}$ & Location of zonal statistics & $\begin{array}{l}\text { [Environmental Layer] } \\
\text { zone_stats.shp }\end{array}$ & $\begin{array}{l}\text { Shapefile containing zonal statistics for each en- } \\
\text { vironmental layer for the specified social value } \\
\text { type or survey subgroup. }\end{array}$ & $\mathrm{X}$ & $\mathrm{X}$ & NA \\
\hline
\end{tabular}



For more information about this publication, contact:

Director, USGS Geosciences and Environmental Change Science Center Denver Federal Center

Building 25

Denver, CO 80225

For additional information, visit: https://www.usgs.gov/centers/gecsc

Publication support provided by the Denver and Rolla Publishing Service Centers 


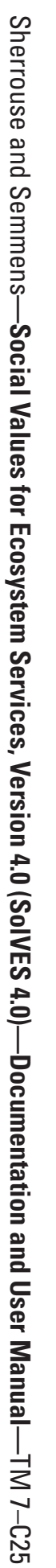

